\title{
Individual, Structural and Exchange Mobility: Decomposition and Axiom based measures
}

\author{
Debasis Sengupta \\ and \\ Nachiketa Chattopadhyay \\ Technical Report No. SOSU/2020/1 \\ 17 April 2020
}

Indian Statistical Institute

Sampling and Official Statistics Unit

Kolkata 700108

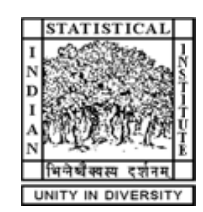




\title{
Individual, Structural and Exchange Mobility: Decomposition and Axiom based measures
}

\author{
Debasis Sengupta and Nachiketa Chattopadhyay \\ Indian Statistical Institute, Kolkata
}

\begin{abstract}
This paper presents a novel and common framework for analysing transition matrices representing social, occupational or economic mobility. It is based on an unambiguous decomposition of structural and exchange (circulation) components of mobility. Each component has a linear representation in terms of elemental movements, which are easy to interpret. These representations are applicable to nominal data as well as ordinal data and continuous data with grouping. Except in the case of nominal data, these lead to different partial orders among transition matrices in respect of individual, exchange and structural mobility. Another benefit ensuing from these representations is a set of directional and other measures of various aspects of mobility. These measures have a common form as sum of coefficients of the linear representations. They can also be derived uniquely from a parsimonious set of sensible axioms. Each measure is scaled conveniently after derivation of sharp lower and upper bounds.
\end{abstract}

KEY WoRDS: transition matrix; decomposition; structural mobility; exchange mobility; partial order; directional indices; measures of mobility

JEL CODE: J62, D63, Z13

\section{Introduction}

Social, occupational and economic mobility has been a topic of sustained interest among social scientists for many decades. The variable under consideration in many of these studies is either naturally discrete (e.g., occupational or social categories, which may be ordered or unordered) or discretized from an originally continuous variable (e.g., income percentile groups). A transition matrix relating movements between two reference periods is often analysed in the light of structural movements manifested by discrepancy between row and column sums (or initial and final state distribution), or association between initial and final states.

There have been persistent attempts to formalize a specific type of mobility, termed as structural mobility that is manifested by non-concurrence between marginal sums of a transition matrix. In contrast, mobility associated with identical row and column sums has been called exchange mobility or circulation mobility (Markandya, 1982). Distinction between these two types of mobility has also been made in the context of income mobility, 
where the income variable is continuous (Fields and Ok, 1996; Ruiz-Castillo, 2004). Any attempt to decompose mobility into structural and exchange components is complicated by the fact that even a manifestly structural movement may have a hidden exchange component that is hard to expunge in an unambiguous manner. This confounding led Sobel (1983) to lament mobility researchers' failure to conceptualize structural and circulation mobility meaningfully or decompose mobility successfully into structural and circulatory components, and to recommend "abandonment of the "structure vs. circulation' framework". Yet subsequent researchers, including Sobel himself, continued to pursue delineation of the two types of mobility (Sobel et al., 1998).

Another line of research has evolved around log-linear (see Goodman, 1979; Breiger, 1981; Hope, 1982) and other non-linear (see Sobel et al., 1998) models of association. The simplest model is that of independence of row and column counts, referred to by Sobel (1983) as perfect mobility (Shorrocks, 1978, Swift, 2004, and many others used this term to mean a special case of the independence model, where the destination distribution is uniform). If this model is fitted to an observed transition matrix, the residual matrix represents association. On the other hand, having constant (zero) row and column sums, this residual matrix also represents a form of exchange mobility. Thus, association models seek to explain a part of exchange mobility.

In the case of a continuous variable such as income, the copula framework can be used to neatly separate association from the marginal distributions of initial and final income, so that these two distributions take care of structural mobility and the copula represents exchange mobility (e.g., Aaberge et al., 2018; Chetty et al., 2017). For discretized variables however, the copula is generally not unique (Richey and Rosberg, 2018). For ordinal or nominal data (such as those describing social and occupational mobility), the copula approach is generally inapplicable.

We present in this paper a fresh approach to fill this gap. Disregarding Sobel's (1983) recommendation, we decompose mobility into structural and exchange components and reap the ensuing benefits. Our point of departure is the fact that the confusion about structural movements (Hope, 1982; Sobel, 1983) lies in stark contrast with the clarity existing in the literature about exchange movements. There is no contradiction among the notions of the latter found in the literature. All exchange movements belong to a vector space spanned by what we call elemental Pure Exchange Movements (PEMs) - pairs of elementary row-column exchanges that do not change any marginal count of a transition matrix. By using the concept of orthogonality of matrices, we show how one can rid any transition matrix of its exchange movement content. This remainder is found to have, as its basis, a set of elemental Structural Change Movements (SCMs), which are swaps of a common size across all rows of a pair of columns of a transition ma- 
trix. Elemental PEMs and SCMs are themselves combinations of elemental individual movements (EIMs), which are single intra-row swaps.

A novel aspect of this vector space approach is that it is applicable to any type of data, be it nominal, ordinal or continuous (after suitable grouping). Another interesting feature is that the transition matrix is viewed as a deviation from a reference transition matrix, which represents an ideal situation, such as that of perfect immobility or perfect mobility. In the first of these two cases the deviation matrix embodies the extent of mobility, while in the second case it represents imperfection in mobility. This deviation matrix is further decomposed into exchange and structural components.

The decomposition produces further dividends. The structural and exchange components are found to be expressed in terms of their respective bases, i.e., linear combinations of elemental PEMs and SCMs. The signed coefficients induce partial orders, in the spirit of Atkinson (1981), Dardanoni (1993), Formby et al. (2004) and others, among matrices in respect of structural and exchange components of their movements.

Linear combinations of these coefficients also lead to a family of simple and intuitively appealing measures for various aspects of mobility and different types of data. It may be recalled that many measures of mobility have been proposed in the literature on the basis of transition matrix, including a function of the trace of the transition matrix (Prais, 1955; Bibby, 1975), its determinant (Shorrocks, 1978) and its second largest eigenvalue (Sommers and Conlisk, 1979), among others (Ferretti and Ganugi, 2013). An often cited measure had been proposed by Bartholomew (1973), who assumed an order among the states and equal separation among successive states. For continuous variables such as income, mobility measures are often derived axiomatically (see Fields and Ok, 1996; Mitra and Ok, 1998; D'Agostino and Dardanoni, 2009; Cowell and Flachaire, 2018). All our measures for discrete variables are developed axiomatically, and some of them reduce to existing measures. A measure of overall mobility in the population is regarded as aggregation of measures of EIM, elemental PEMs or SCMs, which are characterized through a unified framework of parsimonious axioms. The framework works for ordinal data as well as nominal data. In the former case, we propose directional and additively decomposable measures associated with different types of movements. Further, we scale all the proposed measures by deriving sharp upper and lower bounds on their values. Most of the aggregated measures are available in explicit form. For the others, we provide a simple computational algorithm by mapping the relevant optimization problem with least absolute deviation (LAD) regression.

In Section 2, we define the different kinds of movements and present the key decomposition for delineating structural and exchange movements. In Section 3 we present a few partial orders that can be constructed from these decompositions. In Sections 4 and 5, we provide axiomatic characterizations of the measures that follow naturally from the decompositions, in cases of 
ordinal and nominal data, respectively. We provide some concluding remarks in Section 6. Proofs of all the results are given in the appendix.

\section{Movements and decompositions}

\section{$2.1 \quad$ Individual movements}

Consider a population of $n$ individuals who are observed over two points of time. Each individual has an ordered pair of states $(i, j)$, where $i$ is the initial (source) state and $j$ is the final (destination) state. Both $i$ and $j$ are elements of the set $S=\{1,2, \ldots, K\}, K$ being the total number of states, which can be social, occupational, economic or other categories that can be ordered or unordered. The observed transition matrix of counts of movements is the matrix $\boldsymbol{N}$ having $n_{i j}$ as its $(i, j)$ th elements for $i, j=1,2, \ldots, K$.

The row sums of the matrix $\boldsymbol{N}$, denoted by $n_{i} .=\sum_{j=1}^{K} n_{i j}, i=1,2, \ldots, K$, represent the frequency distribution of the population over the states before transition. The distribution after transition is represented by the column sums, $n_{\cdot j}=\sum_{i=1}^{K} n_{i j}, j=1,2, \ldots, K$. The total number of individuals before and after transition is $n=\sum_{i=1}^{K} \sum_{j=1}^{K} n_{i j}=\sum_{j=1}^{K} n_{\cdot j}=\sum_{i=1}^{K} n_{i}$.

Suppose $\boldsymbol{N}_{0}$ is a hypothetical transition matrix that represents the immobile situation, i.e., every individual staying in their respective categories. Thus,

$$
\boldsymbol{N}_{0}=\left(\begin{array}{cccc}
n_{1} & 0 \cdots & 0 & \\
0 & n_{2} & \cdots & 0 \\
\vdots & \vdots & \ddots & \vdots \\
0 & 0 & \cdots & n_{K}
\end{array}\right)
$$

The matrix $\boldsymbol{N}-\boldsymbol{N}_{0}$ has row sums equal to zero. In other words, one can reach from $\boldsymbol{N}_{0}$ to $\boldsymbol{N}$ by redistributing the row sums among the respective rows. This action in respect of row $i$ would change the destination states of some individuals whose source states had been $i$. The simplest step in such a redistribution is one where a single individual originating from source state $i$ is moved from an initial destination state $j$ to the another destination state $j+k$. We can represent this step, which we call an Elementary Individual Movement (EIM), in matrix notations as

$$
\boldsymbol{K}_{i j k}=-\boldsymbol{e}_{i} \boldsymbol{e}_{j}^{\prime}+\boldsymbol{e}_{i} \boldsymbol{e}_{j+k}^{\prime},
$$

for $k=1, \ldots, K-j, j=1, \ldots, K-1, i=1, \ldots, K$, where $\boldsymbol{e}_{1}, \boldsymbol{e}_{2}, \ldots, \boldsymbol{e}_{K}$ are the successive columns of the $K \times K$ identity matrix. It seems possible that any matrix of the form $\boldsymbol{N}-\boldsymbol{N}_{0}$ can be expressed in terms of these EIM matrices as

$$
\boldsymbol{N}-\boldsymbol{N}_{0}=\sum_{i=1}^{K} \sum_{j=1}^{K-1} \sum_{k=1}^{K-j} \xi_{i j k} \boldsymbol{K}_{i j k},
$$


where the $\xi_{i j k} \mathrm{~s}$ are real valued coefficients. However, such a representation would not be unique, as there are $K^{2}(K-1) / 2$ summand matrices compared to the $K(K-1)$ required for unique representation (considering the $K^{2}$ elements and the $K$ constraints on the row sums.) Therefore, we can use a simpler set of EIMs represented by

$$
\boldsymbol{G}_{i j}=-\boldsymbol{e}_{i} \boldsymbol{e}_{j}^{\prime}+\boldsymbol{e}_{i} \boldsymbol{e}_{j+1}^{\prime} \quad \text { for } i=1,2, \ldots, K, j=1,2, \ldots, K-1 .
$$

The number of EIM matrices of the above kind is $K(K-1)$, which is exactly the same as the requisite number mentioned above. Our first theorem shows that $\boldsymbol{N}-\boldsymbol{N}_{0}$ can indeed be represented uniquely as a linear combination of these EIMs.

Theorem 2.1. Let $\boldsymbol{N}$ be a transition matrix with row sums $n_{1}, n_{2} ., \ldots$, $n_{K}$, and $\boldsymbol{N}_{0}$ be the diagonal matrix with these row sums as diagonal elements. Then

$$
\boldsymbol{N}=\boldsymbol{N}_{0}+\sum_{i=1}^{K} \sum_{j=1}^{K-1} \eta_{i j} \boldsymbol{G}_{i j}
$$

where $\boldsymbol{G}_{i j}$ s are defined in (4) and the $\eta_{i j}$ s are defined as

$$
\eta_{i j}=-\sum_{l=1}^{j} \boldsymbol{e}_{i}^{\prime}\left(\boldsymbol{N}-\boldsymbol{N}_{0}\right) \boldsymbol{e}_{l} \quad \text { for } i=1,2, \ldots, K \text { and } j=1,2, \ldots, K-1 .
$$

Further, the above expansion of $\boldsymbol{N}$ in terms of $\boldsymbol{N}_{0}$ and EIM transition matrices is unique.

The unique decomposition of $\boldsymbol{N}$ in terms of $\boldsymbol{N}_{0}$ and a linear combination of EIMs, given in Theorem 2.1, can be interpreted as a process of synthesizing the transition matrix $\boldsymbol{N}$. In this synthesis, there is a series of swaps of destination states of groups of individuals who had a common source state. In particular, $\eta_{i j}$ is the number of individuals, having source state $i$, whose destinations were changed from $j$ to $j+1$. This count can be positive or negative. A negative value of $\eta_{i j}$ indicates a total of $\left|\eta_{i j}\right|$ changes of destination from $j+1$ to $j$, in respect of individuals originating from the source state $i$.

Theorem 2.1 shows that the $\boldsymbol{G}_{i j}$ s can act as a linear basis set of all matrices of the form $\boldsymbol{N}-\boldsymbol{N}_{0}$. In fact, these matrices can also be expressed as (3), since one can always write $\boldsymbol{G}_{i j}=\boldsymbol{K}_{i j 1}$. Conversely, one can write

$$
\boldsymbol{K}_{i j k}=\sum_{l=j}^{j+k-1} \boldsymbol{G}_{i l}, k=1, \ldots, K-j, \quad j=1, \ldots, K-1, \quad i=1, \ldots, K .
$$

Thus, the spaces spanned by the two sets of matrices of the type $\boldsymbol{G}_{i j}$ and $\boldsymbol{K}_{i j k}$ are identical. While a representation of $\boldsymbol{N}-\boldsymbol{N}_{0}$ in terms of the $\boldsymbol{G}_{i j \mathrm{~s}}$ is unique according to Theorem 2.1, a representation in terms of the $\boldsymbol{K}_{i j k} \mathrm{~s}$ is not. For example, a special case of (3) is the representation of Theorem 2.1, 


\begin{tabular}{cccccccc}
\hline $\begin{array}{c}\text { Father's status } \\
\text { group }\end{array}$ & \multicolumn{7}{c}{ Subject's status group } \\
\cline { 2 - 8 } & 1 & 2 & 3 & 4 & 5 & 6 & 7 \\
\hline 1 & 50 & 19 & 26 & 8 & 18 & 6 & 2 \\
2 & 16 & 40 & 34 & 18 & 31 & 8 & 3 \\
3 & 12 & 35 & 65 & 66 & 123 & 23 & 21 \\
4 & 11 & 20 & 58 & 110 & 223 & 64 & 32 \\
5 & 14 & 36 & 114 & 185 & 714 & 258 & 189 \\
6 & 0 & 6 & 19 & 40 & 179 & 143 & 71 \\
7 & 0 & 3 & 14 & 32 & 141 & 91 & 106 \\
\hline
\end{tabular}

Table 2.1: Glass and Hall's (1954) British data on occupational mobility over a generation

where $\xi_{i j 1}=\eta_{i j}$ and $\xi_{i j k}=0$ for $k>1$. Another special case occurs with ${ }^{1}$

$$
\xi_{i j k}= \begin{cases}n_{i, i+k} & \text { if } j=i, k=1, \ldots, K-i, i=1, \ldots, K-1 \\ -n_{i, i-k} & \text { if } j=i-k, k=1, \ldots, i-1, i=2, \ldots, K \\ 0 & \text { otherwise }\end{cases}
$$

Example 2.1. Consider the British data on occupational mobility over a generation, published by Glass and Hall (1954), where occupations are divided into seven groups of successively lower social status, namely (1) Professional and high administrative, (2) Managerial and executive, (3) Inspectional, supervisors, and other nonmanual (higher grade), (4) Same (lower grade), (5) Skilled manual and routine grades of nonmanual, (6) Semi-skilled manual, (7) Unskilled manual. The data are reproduced in Table 2.1. While there is well known ambiguity about the meaning of intergenerational mobility (van de Gaer et al., 2001), Goodman (1979) and Duncan (1979) fitted different association models to the data set. Like these and other researchers, we use the data for illustrative purpose.

Values of the $\eta_{i j}$ s defined in Theorem 2.1 for this data set are as follows.

$$
\left(\begin{array}{llllll}
\eta_{11} & \eta_{12} & \eta_{13} & \eta_{14} & \eta_{15} & \eta_{16} \\
\eta_{21} & \eta_{22} & \eta_{23} & \eta_{24} & \eta_{25} & \eta_{26} \\
\eta_{31} & \eta_{32} & \eta_{33} & \eta_{34} & \eta_{35} & \eta_{36} \\
\eta_{41} & \eta_{42} & \eta_{43} & \eta_{44} & \eta_{45} & \eta_{46} \\
\eta_{51} & \eta_{52} & \eta_{53} & \eta_{54} & \eta_{55} & \eta_{56} \\
\eta_{61} & \eta_{62} & \eta_{63} & \eta_{64} & \eta_{65} & \eta_{66} \\
\eta_{71} & \eta_{72} & \eta_{73} & \eta_{74} & \eta_{75} & \eta_{76}
\end{array}\right)=\left(\begin{array}{rrrrrr}
79 & 60 & 34 & 26 & 8 & 2 \\
-16 & 94 & 60 & 42 & 11 & 3 \\
-12 & -47 & 233 & 167 & 44 & 21 \\
-11 & -31 & -89 & 319 & 96 & 32 \\
-14 & -50 & -164 & -349 & 447 & 189 \\
0 & -6 & -25 & -65 & -244 & 71 \\
0 & -3 & -17 & -49 & -190 & -281
\end{array}\right) .
$$

The negative terms signify single-step upward movements. For higher source states, these movements are outnumbered by single-step downward movements that have positive sign.

\footnotetext{
${ }^{1}$ In this paper we use a comma to separate consecutive subscripts (as in $\eta_{i, j-1}$ ) only when a separator is needed to avoid ambiguity.
} 


\subsection{Pure exchange movements}

Consider the special case of a transition matrix with column sums identical to row sums. In such a case $\boldsymbol{N}-\boldsymbol{N}_{0}$ is a matrix having each row sum and each column sum equal to zero. Thus, one can reach from $\boldsymbol{N}_{0}$ to $\boldsymbol{N}$ through movements that have no effect on the marginal counts. Sharpening a term from the existing literature, we refer to this as a pure exchange movement (PEM). The simplest such movement would consist of swapping of destination states of a single individual and reverse swap of the same pair of destination states in respect of another individual, from a different source state. We call this an elemental PEM and denote it mathematically as an increment matrix of the form

$$
\begin{aligned}
\boldsymbol{F}_{i j k l}= & -\boldsymbol{e}_{i} \boldsymbol{e}_{j}^{\prime}+e_{i} \boldsymbol{e}_{j+l}^{\prime}+\boldsymbol{e}_{i+k} \boldsymbol{e}_{j}^{\prime}-\boldsymbol{e}_{i+k} \boldsymbol{e}_{j+l}^{\prime} \\
& \quad \text { for } k=1, \ldots K-i, l=1, \ldots K-j, i, j=1,2, \ldots, K-1,
\end{aligned}
$$

where $\boldsymbol{e}_{1}, \boldsymbol{e}_{2}, \ldots, \boldsymbol{e}_{K}$ are, as in (2). The elemental PEMs combine linearly to produce a variety of matrices of the form

$$
\boldsymbol{M}=\sum_{i=1}^{K-1} \sum_{j=1}^{K-1} \sum_{k=1}^{K-i} \sum_{l=1}^{K-j} \beta_{i j k l} \boldsymbol{F}_{i j k l} .
$$

that have zero row and column sums. The number of unrestricted elements of $\boldsymbol{M}$ is $(K-1)^{2}$, since it has $K^{2}$ elements with $2 K-1$ independent linear restrictions. However, the number of matrices of the form $(5)$ is $\left(\begin{array}{c}K \\ 2\end{array}\right)^{2}$, which is much larger than $(K-1)^{2}$. It should be possible to express matrices of the form (6) in terms of a smaller set of PEMs. For this purpose, we define $(K-1)^{2}$ elemental PEMs as

$$
\boldsymbol{E}_{i j}=\boldsymbol{F}_{i j 11}=-\boldsymbol{e}_{i} \boldsymbol{e}_{j}^{\prime}+\boldsymbol{e}_{i} \boldsymbol{e}_{j+1}^{\prime}+\boldsymbol{e}_{i+1} \boldsymbol{e}_{j}^{\prime}-\boldsymbol{e}_{i+1} \boldsymbol{e}_{j+1}^{\prime} i, j=1,2, \ldots, K-1 .
$$

The next theorem shows that this subset of elemental PEMs is indeed adequate. In this theorem, we have used the notations $\mathbf{1}$ and $\mathbf{0}$ for vectors of size $K$ having all elements equal to 1 and all elements equal to 0 , respectively.

Theorem 2.2. Let $M$ be a $K \times K$ matrix such that $M \mathbf{1}=\boldsymbol{M}^{\prime} \mathbf{1}=\mathbf{0}$. Then $\boldsymbol{M}$ can be written as

$$
\boldsymbol{M}=\sum_{i=1}^{K-1} \sum_{j=1}^{K-1} \alpha_{i j} \boldsymbol{E}_{i j}
$$

where the $\boldsymbol{E}_{i j}$ s are as defined in (7) and the $\alpha_{i j}$ s are defined as

$$
\alpha_{i j}=-\sum_{k=1}^{j} \sum_{l=1}^{j} \boldsymbol{e}_{k}^{\prime} \boldsymbol{M} \boldsymbol{e}_{l}, \quad \text { for } i, j=1,2, \ldots, K-1 .
$$

Further, the above expansion of $\boldsymbol{M}$ is unique. 
Theorem 2.2 shows that the $\boldsymbol{E}_{i j}$ matrices span the space of all $K \times K$ matrices with zero marginal sums. The uniqueness of the expansion shows that the $(K-1)^{2}$ elemental PEMs are indeed a basis set for these matrices. In the special case of $\boldsymbol{M}=\boldsymbol{F}_{i j k l}$, the expansion of Theorem 2.2 simplifies to

$$
\boldsymbol{F}_{i j k l}=\sum_{i^{\prime}=i}^{i+k-1} \sum_{j^{\prime}=j}^{j+l-1} \boldsymbol{E}_{i^{\prime} j^{\prime}} .
$$

\subsection{Structural change movements}

In respect of social transition, a structural change is loosely associated with change in the state distribution. This corresponds to row and column totals of a transition matrix being non-identical. There has been much confusion about the essence of a structural change. On the one hand, any EIM produces a change in the destination state distribution, and thus can be seen as contributing to structural change. On the other hand, an elemental PEM is a combination of EIMs that nullify the effects of one another on the destination state distribution. For this reason, there has been attempts to distinguish structural changes from exchange movements. Hope (1982) claimed to have achieved this, but Sobel (1983) pointed out that his claim was flawed and even asserted that the very goal of achieving this delineation may not be achievable. We would like to show that Sobel (1983) had been overly pessimistic, i.e., the delineation is indeed possible.

A proper definition of structural change has to be built on the premise that it is a structural attribute, rather than an individual one. This premise is not a matter of preference, but rather one of necessity. Since exchange movements involve EIMs of multiple individuals, anything that has to be delineated from exchange movements must necessarily involve multiple individuals too. Thus, we would not even try to identify a 'structural' movement at the individual level.

Consider the transition matrix $\boldsymbol{N}$ and the diagonal matrix $\boldsymbol{N}_{0}$ obtained from its row sums. Theorem 2.1 gives a decomposition of $\boldsymbol{N}-\boldsymbol{N}_{0}$ in terms of $\boldsymbol{G}_{i j} \mathrm{~s}$, which are EIMs consisting of swaps of destination states. Some combinations of these EIMs might constitute PEMs. In fact, in the special case where row and column sums of $\boldsymbol{N}$ are identical, Theorem 2.2 gives an alternative decomposition of $\boldsymbol{N}-\boldsymbol{N}_{0}$ in terms of $\boldsymbol{E}_{i j}$ s. It is easy to see that

$$
\boldsymbol{E}_{i j}=\boldsymbol{G}_{i j}-\boldsymbol{G}_{i+1, j} \text { for } i=1,2, \ldots, K-1 \text { and } j=1,2, \ldots, K \text {. }
$$

For a general $\boldsymbol{N}$ however, Theorem 2.2 is not applicable. This shows that the set of matrices that may be represented as linear combinations of the $\boldsymbol{E}_{i j} \mathrm{~s}$ is more restricted than those representable as linear combinations of the $\boldsymbol{G}_{i j}$ s. Indeed, there are $K(K-1)$ EIMs represented by the $\boldsymbol{G}_{i j}$ s, and only $(K-1)^{2}$ elemental PEMs represented by the $\boldsymbol{E}_{i j}$ s. Thus, there should be $K-1$ linearly independent EIMs that have nothing to do with PEMs. In 
order to isolate such a set of EIMs formally, let us define the inner product of two $K \times K$ matrices $\boldsymbol{A}$ and $\boldsymbol{B}$ as $\langle\boldsymbol{A}, \boldsymbol{B}\rangle=\sum_{i=1}^{K} \sum_{j=1}^{K} a_{i j} b_{i j}$, where $a_{i j}$ and $b_{i j}$ are the $(i, j)$ th elements of $\boldsymbol{A}$ and $\boldsymbol{B}$, respectively. Note that $\langle\boldsymbol{A}, \boldsymbol{A}\rangle$ is the squared Frobenius norm of $\boldsymbol{A}$. It is easy to see that if the matrices $\boldsymbol{A}$ and $\boldsymbol{B}$ are converted to vectors by concatenating their rows (or columns) in a certain order, the usual inner product of these two vectors coincides with $\langle\boldsymbol{A}, \boldsymbol{B}\rangle$. We can define $\boldsymbol{A}$ and $\boldsymbol{B}$ as orthogonal if $\langle\boldsymbol{A}, \boldsymbol{B}\rangle=0$.

A simple set of matrices that represent EIMs delinked with PEMs is

$$
\boldsymbol{H}_{j}=\sum_{i=1}^{K} \boldsymbol{G}_{i j}=\mathbf{1}\left(-\boldsymbol{e}_{j}+\boldsymbol{e}_{j+1}\right)^{\prime}, \quad j=1,2, \ldots, K-1 .
$$

Indeed, it is easy to see that $\left\langle\boldsymbol{E}_{i j}, \boldsymbol{H}_{k}\right\rangle=0$ for $i, j, k=1,2, \ldots, K-1$. Further, for any set of scalar coefficients $\theta_{1}, \theta_{2}, \ldots, \theta_{K-1}, \sum_{j=1}^{K-1} \theta_{j} \boldsymbol{H}_{j}=\mathbf{0}$ implies

$$
\mathbf{0}=\mathbf{1} \sum_{j=1}^{K-1} \theta_{j}\left(-\boldsymbol{e}_{j}+\boldsymbol{e}_{j+1}\right)^{\prime}=\mathbf{1}\left(-\sum_{j=1}^{K-1} \theta_{j} \boldsymbol{e}_{j}^{\prime}+\sum_{j=2}^{K} \theta_{j-1} \boldsymbol{e}_{j}^{\prime}\right),
$$

i.e., $\theta_{1}=\theta_{2}=\cdots=\theta_{K-1}=0$. Thus, the $\boldsymbol{H}_{j}$ s are linearly independent, and can serve as a basis set of those movements that are orthogonal to all PEMs. The matrix $\boldsymbol{H}_{j}$ represents $K$ simultaneous swaps between the destination states $j$ and $j+1$, in respect of one individual from every source state. We shall refer to this set of swaps as an elemental structural change movement (SCM). All combinations of elemental SCMs are SCMs themselves.

The $\boldsymbol{E}_{i j}$ s and $\boldsymbol{H}_{j}$ s together serve as a basis set of all matrices of the form $\boldsymbol{N}-\boldsymbol{N}_{0}$. In this respect, the combination of $\boldsymbol{E}_{i j}$ s and $\boldsymbol{H}_{j}$ s is an alternative to the basis set of $\boldsymbol{G}_{i j}$ s. They should lead to another decomposition of $\boldsymbol{N}-\boldsymbol{N}_{0}$ in the spirit of Theorem 2.1, with the added advantage that there would be no possibility of confounding between the components assigned to PEMs and SCMs. The next theorem gives such a decomposition.

Theorem 2.3. Let $\boldsymbol{N}$ and $\boldsymbol{N}_{0}$ be as in Theorem 2.1. Then $\boldsymbol{N}-\boldsymbol{N}_{0}$ is uniquely decomposable into sums of elemental PEMs and elemental SCMs as

$$
\boldsymbol{N}-\boldsymbol{N}_{0}=\sum_{j=1}^{K-1} \theta_{j} \boldsymbol{H}_{j}+\sum_{i=1}^{K-1} \sum_{j=1}^{K-1} \alpha_{i j} \boldsymbol{E}_{i j}
$$

where

$$
\begin{aligned}
\theta_{j} & =\frac{1}{K} \sum_{j^{\prime}=1}^{j}\left(n_{j^{\prime} \cdot}-n_{\cdot j^{\prime}}\right) \quad \text { for } j=1,2, \ldots, K-1, \\
\alpha_{i j} & =-\sum_{k=1}^{i} \sum_{l=1}^{j} \boldsymbol{e}_{k}^{\prime}\left(\boldsymbol{N}-\boldsymbol{N}_{0}-\boldsymbol{N}_{1}\right) \boldsymbol{e}_{l}, \quad \text { for } i, j=1,2, \ldots, K-1,
\end{aligned}
$$

and $\boldsymbol{N}_{1}=\sum_{j=1}^{K-1} \theta_{j} \boldsymbol{H}_{j}$. 
The $\boldsymbol{H}_{j}$ s are special cases of the broader class of elemental SCM matrices

$$
\boldsymbol{L}_{j k}=\mathbf{1}\left(-\boldsymbol{e}_{j}+\boldsymbol{e}_{j+k}\right), \quad k=1,2, \ldots, K-j, \quad j=1,2, \ldots, K-1 .
$$

The matrix $\boldsymbol{L}_{j k}$ represents change of the destination state from $j$ to $j+k$, in respect of an individual originating from every single source category. In particular, $\boldsymbol{H}_{j}=\boldsymbol{L}_{j 1}$ and $\boldsymbol{L}_{j k}=\sum_{l=j}^{j+k-1} \boldsymbol{H}_{l}$. Thus, the space of the $\left(\begin{array}{c}K \\ 2\end{array}\right)$ elemental SCMs of the form $\boldsymbol{L}_{j k}$ is the same as the space of the $K-1$ elemental SCMs $\boldsymbol{H}_{1}, \ldots, \boldsymbol{H}_{K-1}$. It follows that the matrix $\boldsymbol{N}_{1}$ has many alternative expansions of the form

$$
\boldsymbol{N}_{1}=\sum_{j=1}^{K-1} \sum_{k=1}^{K-j} \phi_{j k} \boldsymbol{L}_{j k},
$$

of which $\sum_{j=1}^{K-1} \theta_{j} \boldsymbol{H}_{j}$ is a special case.

\subsection{Decomposition of transition matrices}

Theorem 2.3 shows that any transition matrix $\boldsymbol{N}$ can be regarded as a composition of two types of movement, beginning with the initial matrix $\boldsymbol{N}_{0}$ given by (1). The part accounting for structural change movements may be written as

$$
\begin{aligned}
\boldsymbol{N}_{1}= & \sum_{j=1}^{K-1} \theta_{j} \boldsymbol{H}_{j} \\
= & \left(\begin{array}{ccccc}
-\theta_{1} & \theta_{1}-\theta_{2} & \cdots & \theta_{K-2}-\theta_{K-1} & \theta_{K-1} \\
-\theta_{1} & \theta_{2}-\theta_{2} & \cdots & \theta_{K-2}-\theta_{K-1} & \theta_{K-1} \\
\vdots & \vdots & \ddots & \vdots & \vdots \\
-\theta_{1} & \theta_{1}-\theta_{2} & \cdots & \theta_{K-2}-\theta_{K-1} & \theta_{K-1}
\end{array}\right) \\
= & \frac{1}{K}\left(\begin{array}{ccccc}
n \cdot 1-n_{1} \cdot & n \cdot 2-n_{2} . & \cdots & n_{\cdot K-1}-n_{K-1} \cdot & n_{\cdot K}-n_{K} \\
n_{\cdot 1}-n_{1} \cdot & n_{\cdot 2}-n_{2} . & \cdots & n_{\cdot K-1}-n_{K-1} & n_{\cdot K}-n_{K} \\
\vdots & \vdots & \ddots & \vdots & \vdots \\
n \cdot 1-n_{1} \cdot & n \cdot 2-n_{2} . & \cdots & n_{\cdot K-1}-n_{K-1} \cdot & n_{\cdot K}-n_{K}
\end{array}\right) .
\end{aligned}
$$

The remaining part, consisting of pure exchange movements, is

$$
\begin{aligned}
\boldsymbol{N}_{2}= & \sum_{i=1}^{K-1} \sum_{j=1}^{K-1} \alpha_{i j} \boldsymbol{E}_{i j} \\
= & \left(\begin{array}{cccc}
n_{11}-\frac{n_{1}+(K-1) n_{1} .}{K} & n_{12}-\frac{n \cdot 2-n_{2} .}{K} & \cdots & n_{1 K}-\frac{n_{\cdot K}-n_{K} .}{K} \\
n_{21}-\frac{n \cdot 1-n_{1 .}}{K} & n_{22}-\frac{n_{2}+(K-1) n_{2} .}{K} & \cdots & n_{2 K}-\frac{n \cdot K_{K}-n_{K} .}{K} \\
\vdots & \vdots & \ddots & \vdots \\
n_{K 1}-\frac{n \cdot 1-n_{1 .}}{K} & n_{K 2}-\frac{n \cdot 2-n_{2} .}{K} & \cdots & n_{K K}-\frac{n_{K}+(K-1) n_{K} .}{K}
\end{array}\right) .
\end{aligned}
$$


Thus, we have the decomposition

$$
\boldsymbol{N}=\boldsymbol{N}_{0}+\boldsymbol{N}_{1}+\boldsymbol{N}_{2},
$$

with the initial matrix $\boldsymbol{N}_{0}$, the SCM deviation matrix $\boldsymbol{N}_{1}$ and the PEM deviation matrix $\boldsymbol{N}_{2}$ given by (1), (15) and (16), respectively. More compact representations of the three matrices are given below.

$\boldsymbol{N}_{0}=\sum_{i=1}^{K}\left(\boldsymbol{e}_{i}^{\prime} \boldsymbol{N} \mathbf{1}\right) \boldsymbol{e}_{i} \boldsymbol{e}_{i}^{\prime}, \boldsymbol{N}_{1}=\frac{1}{K} \mathbf{1 1}^{\prime}\left(\boldsymbol{N}-\boldsymbol{N}_{0}\right), \boldsymbol{N}_{2}=\left(\boldsymbol{I}-\frac{1}{K} \mathbf{1 1}^{\prime}\right)\left(\boldsymbol{N}-\boldsymbol{N}_{0}\right)$

The transition matrix $\boldsymbol{N}$ has $K^{2}$ elements describing counts of individual movements that can take any value. The matrix $\boldsymbol{N}_{0}$ has $K$ non-zero elements describing row sums of $\boldsymbol{N}$, which have nothing to do with mobility. The matrix $\boldsymbol{N}_{1}$ has $K-1$ free elements describing structural change, while the matrix $\boldsymbol{N}_{2}$ has $(K-1)^{2}$ free elements describing pure exchanges.

Example 2.2. For the British data on occupational mobility over a generation (see Table 2.1, the values of $\theta_{1}, \ldots, \theta_{6}$ defined in (11) are 3.71, $2.43,4.57,13.00,24.57$ and 5.28 , respectively. The values of $\alpha_{i j}$ defined in Theorem 2.3 are as follows.

$$
\left(\begin{array}{llllll}
\alpha_{11} & \alpha_{12} & \alpha_{13} & \alpha_{14} & \alpha_{15} & \alpha_{16} \\
\alpha_{21} & \alpha_{22} & \alpha_{23} & \alpha_{24} & \alpha_{25} & \alpha_{26} \\
\alpha_{31} & \alpha_{32} & \alpha_{33} & \alpha_{34} & \alpha_{35} & \alpha_{36} \\
\alpha_{41} & \alpha_{42} & \alpha_{43} & \alpha_{44} & \alpha_{45} & \alpha_{46} \\
\alpha_{51} & \alpha_{52} & \alpha_{53} & \alpha_{54} & \alpha_{55} & \alpha_{56} \\
\alpha_{61} & \alpha_{62} & \alpha_{63} & \alpha_{64} & \alpha_{65} & \alpha_{66}
\end{array}\right)=\left(\begin{array}{rrrrrr}
75.3 & 57.6 & 29.4 & 13 & -16.6 & -3.3 \\
55.6 & 149.1 & 84.9 & 42 & -30.1 & -5.6 \\
39.9 & 99.7 & 313.3 & 196 & -10.7 & 10.1 \\
25.1 & 66.3 & 219.7 & 502 & 60.7 & 36.9 \\
7.4 & 13.9 & 51.1 & 140 & 483.1 & 220.6 \\
3.7 & 5.4 & 21.6 & 62 & 214.6 & 286.3
\end{array}\right) .
$$

The matrices $\boldsymbol{N}_{1}$ and $\boldsymbol{N}_{2}$ are given below.

$$
\begin{aligned}
& \boldsymbol{N}_{1}=\left(\begin{array}{rrrrrrr}
-3.71 & 1.29 & -2.14 & -8.43 & -11.57 & 19.29 & 5.29 \\
-3.71 & 1.29 & -2.14 & -8.43 & -11.57 & 19.29 & 5.29 \\
-3.71 & 1.29 & -2.14 & -8.43 & -11.57 & 19.29 & 5.29 \\
-3.71 & 1.29 & -2.14 & -8.43 & -11.57 & 19.29 & 5.29 \\
-3.71 & 1.29 & -2.14 & -8.43 & -11.57 & 19.29 & 5.29 \\
-3.71 & 1.29 & -2.14 & -8.43 & -11.57 & 19.29 & 5.29 \\
-3.71 & 1.29 & -2.14 & -8.43 & -11.57 & 19.29 & 5.29
\end{array}\right) \\
& \boldsymbol{N}_{2}= \\
&\left.\begin{array}{rrrrrrrr}
-75.29 & 17.71 & 28.14 & 16.43 & 29.57 & -13.29 & -3.29 \\
19.71 & -111.29 & 36.14 & 26.43 & 42.57 & -11.29 & -2.29 \\
15.71 & 33.71 & -277.86 & 74.43 & 134.57 & 3.71 & 15.71 \\
14.71 & 18.71 & 60.14 & -399.57 & 234.57 & 44.71 & 26.71 \\
17.71 & 34.71 & 116.14 & 193.43 & -784.43 & 238.71 & 183.71 \\
3.71 & 4.71 & 21.14 & 48.43 & 190.57 & -334.29 & 65.71 \\
3.71 & 1.71 & 16.14 & 40.43 & 152.57 & 71.71 & -286.29
\end{array}\right) .
\end{aligned}
$$


It may be noticed that the largest structural change occurs in group 6 (semiskilled manual), where there has been an average increase of 19.29 for every source group, mostly at the expense of groups 5 (Skilled manual and routine grades of nonmanual) and 4 (Inspectional, supervisors, and other nonmanual, of lower grade). Overall, there is a noticeable shift from higher ranked occupations to lower ranked occupations. The matrix $\boldsymbol{N}_{2}$ representing exchange mobility shows a general trend of movement away from the diagonal positions, which indicate strong discordance between occupations across generations.

\subsection{Movements towards imperfection}

As mentioned in Section 1, many researchers (e.g., Shorrocks, 1978; Swift, 2004) used the term perfect mobility to refer to a transition where all the destination distributions are uniform (a more general definition was used by Sobel, 1983). Any transition matrix not having this property is imperfect. For any transition matrix $\boldsymbol{N}$ there is a corresponding matrix $\frac{1}{K} \boldsymbol{N} \mathbf{1 1}{ }^{\prime}$ with the same distribution of source states, which describes perfect mobility. Thus, the difference matrix $\boldsymbol{N}-\frac{1}{K} \boldsymbol{N 1 1 ^ { \prime }}$ represents imperfection in mobility. This matrix has zero row sums, like the matrix $\boldsymbol{N}-\boldsymbol{N}_{0}$ in Section 2.1. Therefore, following Theorem 2.1, we can express it uniquely in terms of elemental EIMs as

$\boldsymbol{N}-\frac{1}{K} \boldsymbol{N} \mathbf{1 1}{ }^{\prime}=\sum_{i=1}^{K} \sum_{j=1}^{K-1} \eta_{i j} \boldsymbol{G}_{i j}, \quad$ where $\left.\eta_{i j}=-\sum_{k=1}^{j} \boldsymbol{e}_{i}^{\prime}\left(\boldsymbol{N}-\frac{1}{K} \boldsymbol{N} \mathbf{1 1}\right)^{\prime}\right) \boldsymbol{e}_{k}$,

for $i=1, \ldots, K, j=1, \ldots, K-1$. A non-unique representation

$$
\boldsymbol{N}-\frac{1}{K} \boldsymbol{N} \mathbf{1 1} 1^{\prime}=\sum_{i=1}^{K} \sum_{j=1}^{K-1} \sum_{k=1}^{K-j} \xi_{i j k} \boldsymbol{K}_{i j k}
$$

as in (3) is also possible. The structural part of this matrix is

$$
\mathbf{N}_{S}=\frac{1}{K} \mathbf{1 1}^{\prime}\left(\mathbf{N}-\frac{1}{K} \mathbf{N} \mathbf{1 1 ^ { \prime }}\right)
$$

which can be expressed in terms of $\boldsymbol{H}_{j} \mathrm{~s}$, as $\boldsymbol{N}_{1}$ had been expressed in Theorem 2.3, or in terms of $\boldsymbol{L}_{j k} \mathrm{~s}$, as in (14). Likewise, the exchange part,

$$
\boldsymbol{N}_{E}=\left(\boldsymbol{I}-\frac{1}{K} \mathbf{1 1}^{\prime}\right) \boldsymbol{N}\left(\boldsymbol{I}-\frac{1}{K} \mathbf{1 1}^{\prime}\right)
$$

can be expressed in terms of elemental PEMs, as in Theorem 2.2 or (6).

Example 2.3. Consider the inter-generational mobility data of Table 2.1. The values of the $\eta_{i j}$ s defined in (18) for this data set are given 
below.

$$
\left(\begin{array}{ccc}
\eta_{11} & \cdots & \eta_{16} \\
\vdots & \ddots & \vdots \\
\eta_{61} & \cdots & \eta_{66} \\
\eta_{71} & \cdots & \eta_{76}
\end{array}\right)=\left(\begin{array}{rrrrrr}
-50.0 & -68.9 & -94.9 & -102.9 & -120.8 & -126.8 \\
-16.0 & -55.9 & -89.9 & -107.8 & -138.8 & -146.7 \\
-11.9 & -46.8 & -111.7 & -177.6 & -300.5 & -323.4 \\
-10.9 & -30.7 & -88.6 & -198.4 & -421.3 & -485.1 \\
-13.6 & -49.1 & -162.7 & -347.3 & -1060.8 & -1318.4 \\
0.1 & -5.7 & -24.6 & -64.5 & -243.3 & -386.2 \\
0.1 & -2.8 & -16.7 & -48.6 & -189.4 & -280.3
\end{array}\right) .
$$

The large negative values in the middle and right side of the matrix indicate that movement away from perfect mobility is dominated by one-step upward movements of many younger generation individuals at the lower end of the occupational pegging order, where the corresponding older generation had been in the middle level of the occupational categories.

\section{Partial orders in the case of ordinal data}

Suppose the transitions concern states that are ordered by the index from superior to inferior. This convention has been followed widely in the literature (see, e.g., Bartholomew (1973), Breiger (1981), Erikson et al. (1979) and Sobel et al. (1998)).

Let $\boldsymbol{N}_{a}$ and $\boldsymbol{N}_{b}$ be two transition matrices with immobile versions $\boldsymbol{N}_{a 0}$ and $\boldsymbol{N}_{b 0}$, respectively, and total numbers of individuals $n_{a}$ and $n_{b}$, respectively. Further, suppose $\boldsymbol{P}_{a}=\frac{1}{n_{a}}\left(\boldsymbol{N}_{a}-\boldsymbol{N}_{a 0}\right)$ and $\boldsymbol{P}_{b}=\frac{1}{n_{b}}\left(\boldsymbol{N}_{b}-\boldsymbol{N}_{b 0}\right)$ represent scaled versions of the movements inherent in $\boldsymbol{N}_{a}$ and $\boldsymbol{N}_{b}$, respectively.

It follows from Theorem 2.1 that the matrix $\boldsymbol{P}_{b}-\boldsymbol{P}_{a}$ can be represented as $\sum_{i=1}^{K} \sum_{j=1}^{K-1} \eta_{i j} \boldsymbol{G}_{i j}$, for some uniquely defined coefficients. We can say that $\boldsymbol{N}_{a}$ represents greater upward movement of individuals than $\boldsymbol{N}_{b}$ (written as $\boldsymbol{N}_{b} \preceq_{i} \boldsymbol{N}_{a}$ ) if the $\eta_{i j}$ 's in the expansion of $\boldsymbol{P}_{b}-\boldsymbol{P}_{a}$ are all nonnegative. It is easy to see that this is indeed a partial order.

Similarly, it follows from Theorem 2.3 that the matrix $\frac{1}{K} \mathbf{1 1}^{\prime}\left(\boldsymbol{P}_{b}-\boldsymbol{P}_{a}\right)$, which is the difference between scaled versions of the structural parts of $\boldsymbol{N}_{b}$ and $\boldsymbol{N}_{a}$, can be expressed as $\sum_{j=1}^{K-1} \theta_{j} \boldsymbol{H}_{j}$, for some uniquely defined coefficients. If all these coefficients are nonnegative, we can say that $\boldsymbol{N}_{a}$ represents greater upward structural movement than $\boldsymbol{N}_{b}$ (written as $\boldsymbol{N}_{b} \preceq_{s}$ $\boldsymbol{N}_{a}$ ). This is also a partial order, as can be verified easily.

A similar partial order can be defined for the exchange part of transition matrices. In order to make sense of the directionality in this context, we consider the bivariate distribution corresponding to a transition matrix and link it with a concept in the literature on income mobility. Suppose $I$ and $J$ are the random variables representing the state of a randomly selected individual before and after a transition, respectively, with probability 
distribution functions

$$
\begin{aligned}
& F(i)=P(I \leq i)=\sum_{i^{\prime}=1}^{i} \frac{n_{i^{\prime}}}{n}, \quad i=1,2, \ldots, K, \\
& G(j)=P(J \leq j)=\sum_{j^{\prime}=1}^{j} \frac{n_{\cdot j^{\prime}}}{n}, \quad j=1,2, \ldots, K .
\end{aligned}
$$

The bivariate distribution of $I$ and $J$ can be identified as

$$
H(i, j)=P(I \leq i, J \leq j)=\sum_{i^{\prime}=1}^{i} \sum_{j^{\prime}=1}^{j} \frac{n_{i^{\prime} j^{\prime}}}{n}, \quad i, j=1,2, \ldots, K .
$$

Let $\Gamma(F, G)$ be the class of all bivariate probability distributions with given marginal distribution functions $F$ and $G$. Suppose $H^{(1)}, H^{(2)} \in \Gamma(F, G)$. In the case of real-valued random variables, Tchen (1980) called $H^{(1)}$ less concordant than $H^{(2)}$ when $H^{(1)}(x, y) \leq H^{(2)}(x, y)$ for $-\infty<x, y<\infty$. Cambanis et al. (1976) and Tchen (1980) showed that this order exists if and only if $H^{(2)}$ can be obtained from $H^{(1)}$ by a finite sequence of concordance increasing change (CIC), where a CIC consists of addition of probability masses of equal magnitude to two corners of a rectangle along its main diagonal and subtraction of the same amount of probability masses from the other two opposite corners of that rectangle. In the case of ordinal data, a CIC can be immediately associated with an elemental PEM (or a larger rectangle formed by sum of several elemental PEMs) with negative multiplier. Addition of one or more CICs to a transition matrix would make it more concordant (i.e., source and destination states are better aligned). The opposite action makes a transition matrix more discordant, i.e., enhances mobility.

It follows from Theorem 2.2 that the difference between scaled versions of the exchange parts of $\boldsymbol{N}_{b}$ and $\boldsymbol{N}_{a}$, namely $\left(\boldsymbol{I}-\frac{1}{K} \mathbf{1 1}^{\prime}\right)\left(\boldsymbol{P}_{b}-\boldsymbol{P}_{a}\right)$, can be written as a linear combination of the $\boldsymbol{E}_{i j}$ s with uniquely defined coefficients. We can say that $\boldsymbol{N}_{b}$ is more discordant than $\boldsymbol{N}_{a}$ (written as $\boldsymbol{N}_{a} \preceq_{e} \boldsymbol{N}_{b}$ ) if all the coefficients in this linear representation are nonnegative.

For any two transition matrices $\boldsymbol{N}_{a}$ and $\boldsymbol{N}_{b}$, we can define scaled versions of movements towards imperfection (see Section 2.5) inherent in them as

$$
\begin{aligned}
\boldsymbol{P}_{a} & =\frac{1}{n_{a}}\left(\boldsymbol{N}_{a}-\frac{1}{K} \boldsymbol{N}_{a} \mathbf{1} \mathbf{1}^{\prime}\right), \\
\boldsymbol{P}_{b} & =\frac{1}{n_{b}}\left(\boldsymbol{N}_{b}-\frac{1}{K} \boldsymbol{N}_{b} \mathbf{1 1}^{\prime}\right) .
\end{aligned}
$$

By using the expansions of $\boldsymbol{P}_{b}-\boldsymbol{P}_{a}$ or its projections in terms of EIM, elemental SCMs and elemental PEMs, we can define partial orders for the individual, structural and exchange parts, respectively, of the movements towards imperfection inherent in the transition matrices $\boldsymbol{N}_{a}$ and $\boldsymbol{N}_{b}$. 


\section{Measures for ordinal data with equi-spaced states}

The partial orders described in Section 3 were based on signs of coefficients of various expansions in terms of elemental matrices. In general one transition matrix may not be ordered with respect to another when these coefficients differ in sign. A complete order can be invoked if one uses the sums of the coefficients as measures.

The sum of various coefficients in the decomposition of a transition matrix would be natural measures of different aspects of mobility. This is because an expansion can be interpreted as a sequence of elemental movements, with each step contributing equally to the overall measure. We would now show that these natural measures also arise as unique consequences of a few intuitively sensible axioms. These measures are also consistent with relevant partial orders in the sense that wherever the partial order is applicable the complete order implied by the corresponding measures is in the same direction.

\subsection{The axiomatic framework}

Keeping in view the decompositions presented in this paper, we would present desirable properties that apply to matrices that represent multiples of elemental movements, and further properties that apply to linear combinations of these matrices. We would use the generic notation $m$ for a measure applicable to both kinds of matrices.

If there are multiple elemental movements of the same type and involving the same states, then a measure of that set of movements should change proportionately with the number of movements. This property is formalized in our first axiom.

Linear Homogeneity (LH). If $\boldsymbol{M}$ is a matrix representing a multiple of an elemental movement (individual, structural or exchange), the measure $m$ satisfies $m(a \boldsymbol{M})=a m(\boldsymbol{M})$ for any positive constant $a$.

Whether directionality should matter is an important consideration for a measure. If it is not, sign reversal of a matrix representing elemental movement should not alter the measure, as postulated below.

Symmetry (SYM). If the matrix $\boldsymbol{M}$ represents a multiple of an elemental movement (individual, structural or exchange), the measure $m$ satisfies $m(-\boldsymbol{M})=m(\boldsymbol{M})$.

On the contrary, if the direction of movement matters, then there ought to be a convention about what constitutes the 'positive' direction. The next axiom chooses these conventions separately for individual, structural and exchange movements, to associate positive signs with 'greater upward 
movement of individuals', 'greater upward structural movement' and 'less concordance', respectively (see Section 3). In the following, the notations $\boldsymbol{K}_{i j k}, \boldsymbol{L}_{j k}$ and $\boldsymbol{F}_{i j k l}$ are used as per (2), (13) and (5), respectively.

Direction (DR). Depending on the context, the measure $m$ satisfies one of the three conditions

(a) $m\left(-\boldsymbol{K}_{i j k}\right)=-m\left(\boldsymbol{K}_{i j k}\right)>0$ for $k=1, \ldots, K-j, j=1, \ldots, K-1$, $i=1, \ldots, K$,

(b) $m\left(-\boldsymbol{L}_{j k}\right)=-m\left(\boldsymbol{L}_{j k}\right)>0$ for $k=1, \ldots, K-j, j=1, \ldots, K-1$,

(c) $m\left(-\boldsymbol{F}_{i j k l}\right)=-m\left(\boldsymbol{F}_{i j k l}\right)<0$ for $k=1, \ldots, K-i, l=1, \ldots, K-j$ and $i, j=1, \ldots, K-1$.

Since any movement is ultimately captured through the initial and final states of the individual, we assume that a simple partition of an elemental movement should not produce a different value of the mobility measure. This is formally stated for the three types of elemental movements as follows.

Path Independence (PIN). Depending on the context, the measure $m$ satisfies one of the three conditions

(a) $m\left(\boldsymbol{K}_{i j, k+l}\right)=m\left(\boldsymbol{K}_{i j k}\right)+m\left(\boldsymbol{K}_{i, j+k, l}\right)$ for $l=1, \ldots, K-j-k, k=$ $1, \ldots, K-j-1, j=1, \ldots, K-2, i=1, \ldots, K$,

(b) $m\left(\boldsymbol{L}_{j, k+l}\right)=m\left(\boldsymbol{L}_{j k}\right)+m\left(\boldsymbol{L}_{j+k, l}\right)$ for $l=1, \ldots, K-j-k, k=$ $1, \ldots, K-j-1, j=1, \ldots, K-2$,

(c) $m\left(\boldsymbol{F}_{i j, k+k^{\prime}, l}\right)=m\left(\boldsymbol{F}_{i j k l}\right)+m\left(\boldsymbol{F}_{i+k, j k^{\prime} l}\right)$ for $k^{\prime}=1, \ldots, K-i-k, k=$ $1, \ldots, K-i-1, i=1, \ldots, K-2, l=1, \ldots, K-j$ and $j=1, \ldots, K-1$; and $m\left(\boldsymbol{F}_{i j k, l+l^{\prime}}\right)=m\left(\boldsymbol{F}_{i j k l}\right)+m\left(\boldsymbol{F}_{i, j+l, k l^{\prime}}\right)$ for $k=1, \ldots, K-i, i=$ $1, \ldots, K-1, l^{\prime}=1, \ldots, K-j-l, l=1, \ldots, K-j-1$ and $j=$ $1, \ldots, K-2$.

When the successive states in the ordinal scale are equidistant, the mobility measure of an elemental movement should depend only on the relative positions of the relevant states; the absolute positions should not matter. This is stated for the three types of elemental movements in the next axiom.

Translation Invariance (TI). Depending on the context, the measure $m$ satisfies one of the three conditions

(a) $m\left(\boldsymbol{K}_{i j k}\right)=m\left(\boldsymbol{K}_{11 k}\right)$ for $k=1, \ldots, K-j, j=1, \ldots, K-1, i=$ $1, \ldots, K$

(b) $m\left(\boldsymbol{L}_{j k}\right)=m\left(\boldsymbol{L}_{1 k}\right)$ for $k=1, \ldots, K-j, j=1, \ldots, K-1$,

(c) $m\left(\boldsymbol{F}_{i j k l}\right)=m\left(\boldsymbol{F}_{11 k l}\right)$ for $k=1, \ldots, K-i, l=1, \ldots, K-j, i, j=$ $1, \ldots, K-1$. 
We now list some desirable properties of a measure $m$ of a combination of elemental movements. This measure should be a suitable aggregation of the measures of the component movements, and determined completely by them. This notion is formalized in the following axiom.

Independence of Irrelevant Information (IIR). If $M_{1}+M_{2}+\cdots+$ $\boldsymbol{M}_{r}$ is a sum of (positive or negative) multiples of matrices representing elemental movements, then the measure $m$ of their sum is of the form

$$
m\left(\boldsymbol{M}_{1}+\boldsymbol{M}_{2}+\cdots+\boldsymbol{M}_{r}\right)=g\left(m\left(\boldsymbol{M}_{1}\right), m\left(\boldsymbol{M}_{2}\right), \ldots, m\left(\boldsymbol{M}_{r}\right)\right)
$$

for some real valued function $g$ of $r$ real variables.

We also need to normalize the overall index by requiring that the measure of a sum of multiples of matrices representing elemental movements should be 0 if the measure of every summand is 0 .

Normalization (NOM). If $\boldsymbol{M}_{1}+\boldsymbol{M}_{2}+\cdots+\boldsymbol{M}_{r}$ is a sum of (positive or negative) multiples of matrices representing elemental movements such that $m\left(\boldsymbol{M}_{1}\right)=m\left(\boldsymbol{M}_{2}\right)=\cdots=m\left(\boldsymbol{M}_{r}\right)=0$, then $m\left(\boldsymbol{M}_{1}+\boldsymbol{M}_{2}+\cdots+\boldsymbol{M}_{r}\right)=0$. (If $m$ also has the IIR property for some function $g$, then this is equivalent to $g$ satisfying $g(0,0, \ldots, 0)=0$.)

Suppose there is a change in a particular summand in a sum of multiples of matrices representing elemental movements, which produces an increment in the measure of that summand. Then the measure of the sum should have an increment proportional to the increment of the measure of the summand, irrespective of which summand it is. If another summand has a similar change, the overall mobility should change in a similar fashion. This notion is described in the next axiom.

Equal Relative Increment (ERI). For the function $g$ mentioned in IIR, $g\left(m_{1}, m_{2}, \ldots, m_{i}+\delta, \ldots, m_{r}\right)=g\left(m_{1}, m_{2}, \ldots, m_{i}, \ldots, m_{r}\right)+c \delta$ for some constant $c$, any index $i=1,2, \ldots, r$ and any real $\delta$.

We have seen in Section 2 that a single matrix can be expressed as different linear combinations of matrices representing elemental movements. If the measure of a matrix is a directional one, it should not depend on which linear combination is used. If a measure is insensitive to direction, then it may not be able to factor in any inherent redundancy (e.g., partial cancelation) in the linear combination. In such a case, one should make a distinction between a measure (say, $m$ ) of specific linear combinations and a measure (say, $M$ ) of the matrix that is represented by any of these linear combinations. In order that redundancy is discounted, it is desirable that the measure of the matrix should be the smallest of the measures of its various representations. This notion is formalized below. 
Minimality (MIN). If a matrix $\boldsymbol{M}$ has multiple expressions of the form $\boldsymbol{M}_{1}+\boldsymbol{M}_{2}+\cdots+\boldsymbol{M}_{r}$, where the summands are (positive or negative) multiples of matrices representing elemental movements, then the overall measure $M(\boldsymbol{M})$ of $\boldsymbol{M}$ is the minimum of $m\left(\boldsymbol{M}_{1}+\boldsymbol{M}_{2}+\cdots+\boldsymbol{M}_{r}\right)$ over all possible expressions of $\boldsymbol{M}, m$ being a measure of such linear combinations.

\subsection{Direction-free measures of overall individual mobility}

For an axiomatic development of a measure of overall individual mobility, we would track multiples of EIM matrices of the type $\boldsymbol{K}_{i j k}$ defined in (2), which represents the change in destination state from $j$ to $j+k$ in respect of a single individual with source state $i$, and then linear combinations of such matrices. Characterizations of directional measures of individual and aggregate mobilities are given in the next two theorems.

Theorem 4.1. A measure $m$ of mobility of a multiple of an EIM matrix defined in (2) satisfies LH, SYM, PIN and TI if and only if $m\left(a \boldsymbol{K}_{i j k}\right) \propto|a| k$ for $k=1, \ldots, K-j, j=1, \ldots, K-1, i=1, \ldots, K$, and any constant $a$.

Theorem 4.2. A measure of mobility of a combination of EIMs represented by the weighted sum $\sum_{i=1}^{K} \sum_{j=1}^{K-1} \sum_{k=1}^{K-j} \xi_{i j k} \boldsymbol{K}_{i j k}$ satisfies the IIR, NOM and ERI properties, and also satisfies the LH, SYM, PIN and TI properties in the special case of multiples of EIM matrices, if and only if it is proportional to $\sum_{i=1}^{K} \sum_{j=1}^{K-1} \sum_{k=1}^{K-j} k\left|\xi_{i j k}\right|$.

We now have to deal with the issue of multiple expansions of the matrix $\boldsymbol{N}-\boldsymbol{N}_{0}$ leading to different measures. For example, the matrix $\boldsymbol{N}=\boldsymbol{e}_{1} \boldsymbol{e}_{2}^{\prime}$ produces $\boldsymbol{N}_{0}=e_{1} \boldsymbol{e}_{1}^{\prime}$, which means $\boldsymbol{N}-\boldsymbol{N}_{0}=\boldsymbol{K}_{111}$. The latter matrix can be alternatively expressed as $\boldsymbol{K}_{112}-\boldsymbol{K}_{121}$. The value of a measure, fitting the description of Theorem 4.2, in the case of $\boldsymbol{K}_{112}-\boldsymbol{K}_{121}$ would be three times its value in the case of $\boldsymbol{K}_{111}$. We can discard the larger values and choose the smallest one, by invoking the property MIN. The final characterization of the measure is given in the next theorem.

Theorem 4.3. For a transition matrix $\boldsymbol{N}$ describing movement of $n$ individuals across $K$ ordinal states, let $\boldsymbol{N}_{0}$ and $\sum_{i=1}^{K} \sum_{j=1}^{K-1} \eta_{i j} \boldsymbol{G}_{i j}$ be as in Theorem 2.1.

(a) The minimum value of the measure of Theorem 4.2 , with respect to all possible representations of $\boldsymbol{N}-\boldsymbol{N}_{0}$ of the form (3), is

$$
\min \left\{\sum_{i=1}^{K} \sum_{j=1}^{K-1} \sum_{k=1}^{K-j} k\left|\xi_{i j k}\right|\right\}=\sum_{i=1}^{K} \sum_{j=1}^{K-1}\left|\eta_{i j}\right| .
$$


(b) Bartholomew's measure (Bartholomew, 1973, p24)

$$
M_{B}=\frac{1}{n} \sum_{i=1}^{K} \sum_{j=1}^{K} n_{i j}|i-j|=\frac{1}{n} \sum_{i=1}^{K} \sum_{j=1}^{K-1}\left|\eta_{i j}\right|
$$

is the only measure (subject to scale adjustment) that satisfies MIN, in which the measure of linear combinations of EIMs satisfies IIR, NOM and ERI, and also satisfies LH, SYM, PIN and TI in the special case of multiples of EIM matrices.

\subsection{Directional measures of overall individual mobility}

Once again we regard all EIMs as a collection of elemental EIMs and build the measure starting from the constituents. For a directional measure, we have to replace the property SYM by DR.

Theorem 4.4. A measure $m$ of mobility of a multiple of an EIM matrix defined in (2) satisfies LH, DR, PIN and TI if and only if for any constant $a, m\left(a \boldsymbol{K}_{i j k}\right)$ is a positive constant times $-a k$ for $k=1, \ldots, K-j, j=$ $1, \ldots, K-1, i=1, \ldots, K$.

Theorem 4.5. A measure of mobility of a combination of EIM matrices represented by the weighted sum $\sum_{i=1}^{K} \sum_{j=1}^{K-1} \sum_{k=1}^{K-j} \xi_{i j k} \boldsymbol{K}_{i j k}$ satisfies the IIR, NOM and ERI properties, and also satisfies the LH, DR, PIN and TI properties in the special case of multiples of EIM matrices, if and only if it is a positive constant times $-\sum_{i=1}^{K} \sum_{j=1}^{K-1} \sum_{k=1}^{K-j} k \xi_{i j k}$.

The stage is now set for characterizing an index of overall individual mobility. Contrary to the case of the direction-free measure, we would not need the property MIN. Instead, we now show that the measure already developed above for linear combinations of EIMs is invariant under the representations, and is therefore well defined.

Theorem 4.6. For a transition matrix $\boldsymbol{N}$ describing movement of $n$ individuals across $K$ ordinal states, let $\boldsymbol{N}_{0}$ and $\sum_{i=1}^{K} \sum_{j=1}^{K-1} \eta_{i j} \boldsymbol{G}_{i j}$ be as in Theorem 2.1.

(a) For any representation of $\boldsymbol{N}-\boldsymbol{N}_{0}$ of the form (3),

$$
\sum_{i=1}^{K} \sum_{j=1}^{K-1} \sum_{k=1}^{K-j} k \xi_{i j k}=\sum_{i=1}^{K} \sum_{j=1}^{K-1} \eta_{i j}
$$

(b) The measure due to Chattopadhyay et al. (2019)

$$
M_{S}=\frac{1}{n(K-1)} \sum_{i=1}^{K-1} \sum_{j=i+1}^{K}\left(n_{j i}-n_{i j}\right)(j-i)=-\frac{1}{n(K-1)} \sum_{i=1}^{K} \sum_{j=1}^{K-1} \eta_{i j}
$$


is the only measure lying between -1 and 1 that, irrespective of the representation (3), satisfies the IIR, NOM and ERI properties, and also satisfies the LH, DR, PIN and TI properties in the special case of multiples of EIM matrices.

The extreme values, -1 and 1 , are attained by $M_{S}$ when $\boldsymbol{N}=n \boldsymbol{e}_{1} \boldsymbol{e}_{K}^{\prime}$ and $\boldsymbol{N}=n \boldsymbol{e}_{K} \boldsymbol{e}_{1}^{\prime}$, respectively.

Part (a) of Theorem 4.3 indicates that $\sum_{i=1}^{K} \sum_{j=1}^{K-1} \eta_{i j} \boldsymbol{G}_{i j}$ is the simplest version of all representations of $\boldsymbol{N}-\boldsymbol{N}_{0}$ of the form (3). This fact imparts additional elegance on the signed measure $M_{S}$ defined in (24).

Chattopadhyay et al. (2019) had derived the signed measure $M_{S}$ by removing the absolute value in the expression (23) of $M_{B}$. By splitting the positive and negative parts of $M_{S}$ they had also proposed two measures that can capture the directions of mobility. The first such measure is

$$
M_{B U}=\frac{1}{n} \sum_{i=2}^{K} \sum_{j=1}^{i-1} n_{i j}(i-j) .
$$

Note that $M_{B U}$ essentially captures all upward movements that have taken place in a society and ignores the downward movements. Similarly, a measure of downward mobility is

$$
M_{B D}=\frac{1}{n} \sum_{i=1}^{K-1} \sum_{j=i+1}^{K} n_{i j}(j-i) .
$$

It is easy to see from (23) and (24) that

$$
M_{B}=M_{B U}+M_{B D} \quad \text { and } M_{S}=\frac{1}{K-1}\left(M_{B U}-M_{B D}\right)
$$

It transpires from Theorems 4.3 and 4.6 that the measures $M_{B}$ and $M_{S}$ are expressed in terms of the $\eta_{i j}$ s defined in Theorem 2.1. Since all measures of individual mobility are meant to capture the movements of individuals from their respective initial states, it makes sense that they would be determined entirely by the offset matrix $\boldsymbol{N}-\boldsymbol{N}_{0}$. The next theorem summarizes these characterizations of the two measures (already given in Theorems 4.3 and 4.6) along with those of $M_{B U}$ and $M_{B D}$.

Theorem 4.7. The measures $M_{B}, M_{B U}, M_{B D}$ and $M_{S}$ of overall individual mobility can be expressed as

$$
\begin{aligned}
& M_{B U}=\frac{1}{n} \sum_{i=1}^{K-1} \sum_{\substack{j=1 \\
j<i}}^{K-1}\left|\eta_{i j}\right|=-\frac{1}{n} \sum_{\substack{i=1 \\
j<i}}^{K} \sum_{\substack{j=1 \\
j<i}}^{K-1} \eta_{i j}, \\
& M_{B D}=\frac{1}{n} \sum_{i=1}^{K} \sum_{\substack{j=1 \\
j \geq i}}^{K-1}\left|\eta_{i j}\right|=\frac{1}{n} \sum_{\substack{i=1 \\
j \geq i}}^{K-1} \sum_{\substack{j=1 \\
j \geq i}}^{K-1} \eta_{i j},
\end{aligned}
$$




$$
\begin{aligned}
& M_{B}=\frac{1}{n} \sum_{i=1}^{K} \sum_{j=1}^{K-1}\left|\eta_{i j}\right|, \\
& M_{S}=-\frac{1}{n(K-1)} \sum_{i=1}^{K-1} \sum_{j=1}^{K} \eta_{i j},
\end{aligned}
$$

where the $\eta_{i j}$ s are as defined in Theorem 2.1.

We now turn to the further decomposition of $\boldsymbol{N}-\boldsymbol{N}_{0}$ into SCM and PEM matrices as given in Theorem 2.3. The next theorem gives the expressions of the above four measures in terms of the $\theta_{j}$ s and $\alpha_{i j}$ s.

Theorem 4.8. The measures $M_{B U}, M_{B D}, M_{B}$ and $M_{S}$ of overall individual mobility may be expressed as

$$
\begin{aligned}
M_{B U} & =-\frac{1}{n} \sum_{j=1}^{K-1}(K-j) \theta_{j}+\frac{1}{n} \sum_{i=1}^{K-1} \alpha_{i i}, \\
M_{B D} & =\frac{1}{n} \sum_{j=1}^{K-1} j \theta_{j}+\frac{1}{n} \sum_{i=1}^{K-1} \alpha_{i i}, \\
M_{B} & =\frac{1}{n} \sum_{j=1}^{K-1}\left(\sum_{i=1}^{j} \theta_{j}-\sum_{i=j+1}^{K} \theta_{j}\right)+\frac{2}{n} \sum_{i=1}^{K-1} \alpha_{i i}, \\
M_{S} & =-\frac{K}{n(K-1)} \sum_{j=1}^{K-1} \theta_{j} .
\end{aligned}
$$

The first sum in the expression of $M_{B U}$ in the above theorem may be interpreted as the contribution of SCMs towards the measure of upward mobility, while the second sum may be regarded as the contribution of PEMs. The contribution of SCMs represents the net increment of count effected by all pure SCMs happening in those cases where the index of the initial state $i$ had been greater than or equal to the final state $j$ (i.e., the initial status had been at least as high as the final status). An elemental PEM represented by $\boldsymbol{E}_{i i}$ contributes a single upward movement from state $i$ to $i+1$, while there is zero net contribution from an elemental PEM of the form $\boldsymbol{E}_{i j}$ for $i \neq j$. Therefore $\sum_{i=1}^{K-1} \alpha_{i i}$ is the net increment of count effected by all the elemental PEMs. The expressions for $M_{B D}$ and $M_{B}$ have similar interpretations. All these expressions, with the exception of $M_{S}$, show that aggregate measures of individual mobility generally involve an interplay between SCMs and PEMs, which proved difficult to delineate thus far. However, none of these expressions involves PEMs corresponding to $\alpha_{i j}$ s with $j \neq i$. The expression for $M_{S}$ depends only on the $\theta_{j}$ s, which is why it would be useful as a measure of structural mobility also, as we shall see in Section 4.4. 
Example 4.1. For the occupational mobility data of Table 2.1, the different measures of individual mobility are: $M_{B}=1.0583, M_{S}=-0.0179$, $M_{B U}=0.4756$ and $M_{B D}=0.5828$. The three measures other than $M_{S}$ are on the scale 0 to 6 , while $M_{S}$ is on the scale -1 to 1 . Evidently the extent of downward movement surpasses that of upward movement, producing a net downward mobility captured by $M_{S}$.

\subsection{Directional measure of structural mobility}

Here, we view all SCMs as a collection of elemental SCMs, and develop a measure accordingly.

Theorem 4.9. A measure $m$ of structural mobility of a multiple of an elemental SCM matrix defined in (13) satisfies LH, DR, PIN and TI if and only if $m\left(a \boldsymbol{L}_{j k}\right)=-c a k$ for $k=1, \ldots, K-j, j=1, \ldots, K$ and any real $a$, where $c$ is a positive constant.

Theorem 4.10. A measure of structural mobility of a combination of elemental SCM matrices represented by the weighted sum $\sum_{j=1}^{K-1} \sum_{k=1}^{K-j} \phi_{j k} \boldsymbol{L}_{j k}$ satisfies the IIR, NOM and ERI properties, and also satisfies the LH, DR, PIN and TI properties in the special case of multiples of elemental SCM matrices, if and only if it is a positive constant times $-\sum_{j=1}^{K-1} \sum_{k=1}^{K-j} k \phi_{j k}$.

Theorem 4.11. For a transition matrix $\boldsymbol{N}$, let the $\boldsymbol{N}_{1}=\sum_{j=1}^{K-1} \theta_{j} \boldsymbol{H}_{j}$ be as in Theorem 2.3 and $\sum_{j=1}^{K-1} \sum_{k=1}^{K-j} \phi_{j k} \boldsymbol{L}_{j k}=\boldsymbol{N}_{1}$. Then

(a) $\sum_{j=1}^{K-1} \sum_{k=1}^{K-j} k \phi_{j k}=\sum_{j=1}^{K-1} \theta_{j}$.

(b) $\sum_{j=1}^{K-1}\left|\theta_{j}\right| \leq \sum_{j=1}^{K-1} \sum_{k=1}^{K-j} k\left|\phi_{j k}\right|$.

(c) $\sum_{j=1}^{K-1} \theta_{j}=\frac{1}{K} \sum_{i=1}^{K} i\left(n_{\cdot i}-n_{i}\right)$.

Part (a) of the above theorem shows that a measure satisfying all the conditions of Theorem 4.10 may be regarded as an overall measure of structural mobility (and not just a measure of a particular combination of elemental SCMs). Part (b) shows that even though the specific decomposition of $\boldsymbol{N}_{1}$ is immaterial for computation of the measure, the decomposition $\sum_{j=1}^{K-1} \theta_{j} \boldsymbol{H}_{j}$ is the simplest one in some sense. Part (c) expresses the sum of coefficients of this simplest decomposition in terms of the row and column sums of the transition matrix.

Once $-\sum_{j=1}^{K-1} \theta_{j}$ is recognized as the quantity with which the requisite measure should be proportional, the next issue is that of choosing a suitable scale factor. It follows from Theorem 4.8 that we already have a scaled measure, namely $M_{S}$ defined in (24), which lies between -1 and 1 . Even though 
it was introduced as a signed measure of aggregate individual mobility, it also serves as a signed measure of aggregate structural mobility. In either role it is the only (scaled) measure that satisfies the requisite properties.

If $M_{S}$ is viewed as a measure of structural mobility, it is natural to expect that it should depend on the transition matrix only through its dimension and row and column sums. An explicit expression of $M_{S}$ in terms of these quantities, which transpires from Theorems 4.6 and 4.11, is given below.

$$
M_{S}=\frac{1}{n(K-1)} \sum_{i=1}^{K} i\left(n_{i \cdot}-n_{\cdot i}\right), \text { where } n_{\cdot i}=\sum_{j=1}^{K} n_{j i}, n_{i}=\sum_{j=1}^{K} n_{i j} .
$$

Note that every $\boldsymbol{H}_{j}$ represents a downward movement. Therefore $\theta_{j} \boldsymbol{H}_{j}$ corresponds to an upward movement if $\theta_{j}$ is negative, and to a downward movement if $\theta_{j}$ is positive. We can define a measure of upward structural mobility, $M_{S U}$, and a measure of downward structural mobility, $M_{S D}$, as

$$
\begin{gathered}
M_{S U}=-\frac{K}{n(K-1)} \sum_{\substack{j=1 \\
\theta_{j}<0}}^{K-1} \theta_{j}, \\
M_{S D}=\frac{K}{n(K-1)} \sum_{\substack{j=1 \\
\theta_{j}>0}}^{K-1} \theta_{j} .
\end{gathered}
$$

It transpires that each of these measures lie between 0 an 1 (with the extreme values attained when $\boldsymbol{N}=n \boldsymbol{e}_{1} \boldsymbol{e}_{K}^{\prime}$ or $\boldsymbol{N}=n \boldsymbol{e}_{K} \boldsymbol{e}_{1}^{\prime}$ ), and that $M_{S}=M_{S U}-$ $M_{S D}$. In other wards, $M_{S}$ is a signed measure of net upward mobility.

Now suppose $\theta_{j} \geq 0$ for $j=1,2, \ldots, K-1$, i.e., $M_{S U}=0$. This condition means that for $j=1,2, \ldots, K-1$, we have the inequality

$$
0 \leq \frac{K}{n} \theta_{j}=\frac{K}{n} \sum_{j^{\prime}=1}^{j}\left(\theta_{j^{\prime}}-\theta_{j^{\prime}-1}\right)=\frac{1}{n} \sum_{j^{\prime}=1}^{j} n_{j^{\prime} \cdot}-\frac{1}{n} \sum_{j^{\prime}=1}^{j} n_{\cdot j^{\prime}} .
$$

Thus, the marginal cumulative distributions of the states before and after the transition have a first order stochastic dominance relation, with the post-transition state being stochastically larger. In terms of the partial order defined in Section 3, this condition is equivalent to $\boldsymbol{N}$ having greater downward movement than $\boldsymbol{N}_{0}$, i.e, $\boldsymbol{N} \preceq_{s} \boldsymbol{N}_{0}$, which is much stronger than $M_{S} \leq 0$.

We have already noted that the row and column sums remain unchanged under PEM. Hence The expression (28) implies that the measure $M_{S}$ is invariant under such a transformation.

Example 4.2. For the inter-generational mobility data of Table 2.1, the measure $M_{S}$ of structural mobility had already been computed as -0.0179 (see Example 4.1). When it measure is split into upward and downward components, it turns out that $M_{S U}=0$ and $M_{S D}=0.0179$. 


\subsection{Directional measure of exchange mobility}

Turning to exchange mobility, we now derive a measure for elemental PEMs and seek to aggregate them to derive an overall measure of PEMs in a transition matrix.

Theorem 4.12. A measure $m$ of pure exchange mobility satisfies LH, DR, PIN and TI if and only if it is of the form $m\left(a \boldsymbol{F}_{i j k l}\right)=c a k l$ for $k=$ $1, \ldots, K-i, l=1, \ldots, K-j, i, j=1, \ldots, K-1$, where $c$ is a positive constant.

Theorem 4.13. A measure $m$ of structural mobility of a combination of movements represented by the weighted sum

$$
\sum_{i=1}^{K-1} \sum_{j=1}^{K-1} \sum_{k=1}^{K-i} \sum_{l=1}^{K-j} \beta_{i j k l} \boldsymbol{F}_{i j k l}
$$

satisfies the IIR, NOM and ERI properties, and also satisfies the LH, DR, PIN and TI properties for multiples of elemental PEM matrices, if and only if

$$
m\left(\sum_{i=1}^{K-1} \sum_{j=1}^{K-1} \sum_{k=1}^{K-i} \sum_{l=1}^{K-j} \beta_{i j k l} \boldsymbol{F}_{i j k l}\right)=c \sum_{i=1}^{K-1} \sum_{j=1}^{K-1} \sum_{k=1}^{K-i} \sum_{l=1}^{K-j} \beta_{i j k l}
$$

for some positive constant $c$.

We would now show that a measure fitting the description of Theorem 4.13 does not vary across different decompositions of the form (6) of the exchange part of a transition matrix.

Theorem 4.14. For a transition matrix $\boldsymbol{N}$, suppose $\boldsymbol{N}_{2}$ is the matrix $\sum_{i=1}^{K-1} \sum_{j=1}^{K-1} \alpha_{i j} \boldsymbol{E}_{i j}$ defined in Theorem 2.3, alternatively expressed as

$$
\mathbf{N}_{2}=\sum_{i=1}^{K-1} \sum_{j=1}^{K-1} \sum_{k=1}^{K-i} \sum_{l=1}^{K-j} \beta_{i j k l} \boldsymbol{F}_{i j k l} .
$$

Then

(a) $\sum_{i=1}^{K-1} \sum_{j=1}^{K-1} \sum_{k=1}^{K-i} \sum_{l=1}^{K-j} \beta_{i j k l}=\sum_{i=1}^{K-1} \sum_{j=1}^{K-1} \alpha_{i j}$.

(b) $\sum_{i=1}^{K-1} \sum_{j=1}^{K-1}\left|\alpha_{i j}\right| \leq \sum_{i=1}^{K-1} \sum_{j=1}^{K-1} \sum_{k=1}^{K-i} \sum_{l=1}^{K-j}\left|\beta_{i j k l}\right|$.

(c) $\sum_{i=1}^{K-1} \sum_{j=1}^{K-1} \alpha_{i j}=-\sum_{i=1}^{K} \sum_{j=1}^{K} i j \boldsymbol{e}_{i}^{\prime} \boldsymbol{N}_{2} \boldsymbol{e}_{j}$.

It transpires from Theorem 4.14 that all measures satisfying the conditions of Theorem 4.13 are identical, though the description in terms of the $\alpha_{i j} \mathrm{~s}$ is the simplest. Because of this invariance, such a measure of a linear combination of PEMs represented by $\boldsymbol{N}_{2}$ can be regarded as an overall 
measure of PEM in $\boldsymbol{N}$. Part (c) of the theorem gives an easily computable expression that can be used as a measure after appropriate scaling.

Before looking for suitable scaling of the measure $\sum_{i=1}^{K-1} \sum_{j=1}^{K-1} \alpha_{i j}$ derived above, we would provide some characterizations and interpretations.

It has been observed in Section 3 and also at the beginning of this section that positive sign of an $\alpha_{i j}$ represents a movement that reduces concordance between the initial and the final states. The extent of concordance of the two random variables $I$ and $J$ may be captured by their covariance - a measure of linear association that is a natural choice for equally spaced states.

For the purpose of comparing two transition matrices with fixed marginal counts, $E(I)$ and $E(J)$ would be identical in the two cases. Therefore, it is enough to consider $E(I J)$. The difference between $E(I J)$ under any two transition matrices with common marginal counts would be a measure of the combined effect of all PEMs that account for their difference. The PEM matrix $\boldsymbol{N}_{2}$ can indeed be written as the difference between two transition matrices:

$$
\begin{aligned}
\boldsymbol{N}_{2} & =\left(\boldsymbol{N}+\boldsymbol{N}_{B}\right)-\left(\boldsymbol{N}_{0}+\boldsymbol{N}_{A}\right) \\
\text { where } \boldsymbol{N}_{B} & =\frac{1}{K}\left(\begin{array}{ccccc}
n_{1} \cdot & n_{2} . & \cdots & n_{K-1} & n_{K} \\
n_{1} \cdot & n_{2} . & \cdots & n_{K-1} & n_{K} \\
\vdots & \vdots & \ddots & \vdots & \vdots \\
n_{1} \cdot & n_{2} & \cdots & n_{K-1} & n_{K}
\end{array}\right) \\
\boldsymbol{N}_{A} & =\frac{1}{K}\left(\begin{array}{ccccc}
n_{1} & n_{\cdot 2} & \cdots & n_{\cdot K-1} & n_{\cdot K} \\
n_{\cdot 1} & n_{\cdot 2} & \cdots & n_{\cdot K-1} & n_{\cdot K} \\
\vdots & \vdots & \ddots & \vdots & \vdots \\
n \cdot 1 & n_{\cdot 2} & \cdots & n_{\cdot K-1} & n_{\cdot K}
\end{array}\right)
\end{aligned}
$$

and $\boldsymbol{N}_{A}-\boldsymbol{N}_{B}=\boldsymbol{N}_{1}$. Since $\boldsymbol{N}+\boldsymbol{N}_{B}$ and $\boldsymbol{N}_{0}+\boldsymbol{N}_{A}$ are transition matrices with identical row and column sums, they correspond to distinct bivariate distributions with common marginal distributions. Therefore, the difference of the expected values of $I J$ with respect to these two distributions would be a reasonable measure of the net effect of all the PEMs of $\boldsymbol{N}_{2}$. It is easy to see that the expected value with respect to the first distribution is $E(I J)+E(I)(K+1) / 2$, while that under the second distribution is $E(J)(K+$ $1) / 2+E\left(I^{2}\right)$. Further, the requisite difference simplifies as

$$
\begin{aligned}
E(I J) & +\frac{K+1}{2}(E(I)-E(J))-E\left(I^{2}\right) \\
= & \frac{1}{n}\left[\sum_{i=1}^{K} \sum_{j=1}^{K} i j n_{i j}+\frac{1}{K} \sum_{i=1}^{K} \sum_{j=1}^{K} i j\left(n_{j}-n_{\cdot j}\right)-\sum_{i=1}^{K} i^{2} n_{i}\right] \\
= & \frac{1}{n} \sum_{i=1}^{K} \sum_{j=1}^{K} i j \boldsymbol{e}_{i}^{\prime}\left(\boldsymbol{N}-\boldsymbol{N}_{0}-\boldsymbol{N}_{1}\right) \boldsymbol{e}_{j}=\frac{1}{n} \sum_{i=1}^{K} \sum_{j=1}^{K} i j \boldsymbol{e}_{i}^{\prime} \boldsymbol{N}_{2} \boldsymbol{e}_{j} .
\end{aligned}
$$


In other words, this difference of expected values is a measure of concordance or association (i.e., a negative measure of discordance or mobility).

We now summarize the above findings through multiple forms of a single measure of mobility, and justify its scaling through the following theorem.

$$
\begin{aligned}
M_{E} & =-\frac{1}{n}\left(\frac{4}{3(K-1)}\right)^{2} \sum_{i=1}^{K} \sum_{j=1}^{K} i j \boldsymbol{e}_{i}^{\prime} \boldsymbol{N}_{2} \boldsymbol{e}_{j} \\
& =\frac{1}{n}\left(\frac{4}{3(K-1)}\right)^{2} \sum_{i=1}^{K-1} \sum_{j=1}^{K-1} \alpha_{i j} \\
& =\left(\frac{4}{3(K-1)}\right)^{2}\left[\frac{K+1}{2}(E(J)-E(I))+E\left(I^{2}\right)-E(I J)\right] \\
& =\frac{1}{n}\left(\frac{4}{3(K-1)}\right)^{2}\left[\frac{1}{K} \sum_{i=1}^{K} \sum_{j=1}^{K} i j\left(n_{\cdot j}-n_{j} .\right)+\sum_{i=1}^{K} i^{2} n_{i}-\sum_{i=1}^{K} \sum_{j=1}^{K} i j n_{i j}\right]
\end{aligned}
$$

Theorem 4.15. The following results hold for the measure $M_{E}$ of exchange mobility given by (35).

(a) The measure is bounded from above and below as

$$
-\frac{1}{9} \leq M_{E} \leq \frac{8}{9}
$$

(b) The upper bound is attained for a transition matrix if and only if $n_{1 K}=\lambda n, n_{K 1}=(1-\lambda) n$ for some $\lambda \in[0,1]$ and all other $n_{i j}$ s are 0.

(c) The lower bound is attained for a transition matrix if and only if $n_{(K+3) / 4,1}=\lambda n, n_{(3 K+1) / 4, K}=(1-\lambda) n$ for some $\lambda \in[0,1]$ and all other $n_{i j}$ s are 0 .

Consider two transition matrices with identical marginal counts. In view of the sharp bounds given in Theorem 4.15, the difference between their $M_{E}$ measures can range from -1 to 1 . This justifies the choice of the scaling of $M_{E}$ given in (32).

Recall from Section 3 that negative $\alpha_{i j}$ s correspond to mobility enhancing PEMs (i.e., those enhancing discordance between pre-and post-transition states), whereas positive $\alpha_{i j}$ s correspond to PEMs that reduce mobility. Therefore, we can define a measure of discordance enhancing PEM of a transition matrix as

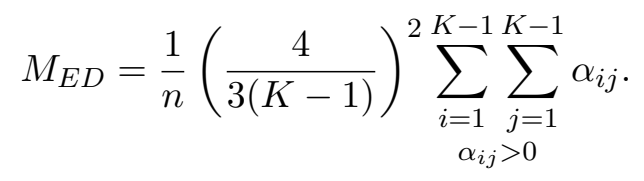


Likewise, a measure of concordance enhancing PEM of a transition matrix is

$$
M_{E C}=-\frac{1}{n}\left(\frac{4}{3(K-1)}\right)^{2} \sum_{\substack{i=1 \\ \alpha_{i j}<0}}^{K-1} \sum_{j=1}^{K-1} \alpha_{i j} .
$$

The measures $M_{E C} M_{E D}$ are both positive. Further,

$$
M_{E}=M_{E D}-M_{E C} .
$$

If $\alpha_{i j} \geq 0$ for $i, j=1,2, \ldots, K-1$, i.e., $M_{E C}=0$, then one can say that $\boldsymbol{N}$ is more discordant than $\boldsymbol{N}_{0}$, i.e., $\boldsymbol{N}_{0} \preceq_{e} \boldsymbol{N}$ (see Section 3). In this case, the pure exchange part of $\boldsymbol{N}$ is entirely discordance enhancing. This condition is much stronger than $M_{E} \geq 0$.

Example 4.3. Consider the inter-generational mobility data of Table 2.1. The measure $M_{E}$ of exchange mobility turns out to be 0.0497 . The measures $M_{E D}$ and $M_{E C}$ of discordance and concordance enhancing exchange mobilities are 0.0506 and 0.0009 , respectively. Thus, most of exchange movements are discordance enhancing.

\subsection{Measures of imperfection in mobility}

As mentioned in Section 2.5, the point of departure of imperfection is the perfect mobility matrix, and the benchmark of perfect mobility for a given transition matrix $\boldsymbol{N}$ is $\frac{1}{K} \boldsymbol{N} \mathbf{1 1}{ }^{\prime}$. A general form of the expansion (19) is

$$
\boldsymbol{N}-\frac{1}{K} \boldsymbol{N} \mathbf{1 1}^{\prime}=\sum_{i=1}^{K} \sum_{j=1}^{K} \sum_{l=1}^{K} \tau_{i j l} \boldsymbol{K}_{i j, l-j},
$$

which is a sum of EIM. We have axiomatically developed in Theorem 4.2 a direction-free measure for combinations of EIMs such as (38). It was also shown in part (a) of Theorem 4.3 that the simplest of all combinations of the type (38), leading to the smallest value of the measure occurs in the special case (18). This result holds for $\boldsymbol{N}-\frac{1}{K} \boldsymbol{N} \mathbf{1 1} \mathbf{1}^{\prime}$ as much as it did for $\boldsymbol{N}-\boldsymbol{N}_{0}$. Therefore, in order to get a measure of imperfection in mobility, we only need to adapt part (b) of Theorem 4.3 to the present situation.

Theorem 4.16. Let a transition matrix $N$, describing the movement of $n$ individuals across $K$ ordinal states, have the expansion (18). Then the only measure of imperfection in individual mobility lying between 0 and 1 that satisfies MIN, in which the measure of linear combinations of EIMs satisfies the IIR, NOM and ERI properties, and also satisfies the LH, SYM, PIN and TI properties in the special case of multiples of EIM matrices, is

$$
M_{I}=\frac{2}{n(K-1)} \sum_{i=1}^{K} \sum_{j=1}^{K}\left|\sum_{k=1}^{j} n_{i k}-\frac{j}{K} n_{i} .=\frac{2}{n(K-1)} \sum_{i=1}^{K} \sum_{j=1}^{K-1}\right| \eta_{i j} \mid .
$$


Further, the measure has the value 0 when $\boldsymbol{N}$ represents perfect mobility, and it attains the value 1 when the destination state is either 1 or $K$ for each individual from every source state.

Recognizing that departures from the ideal case of perfect mobility can be in the upward or the downward direction, we can also develop a signed measure for capturing and delineating these departures. For this purpose, we presume as in Section 4.3 that a state with higher social prestige or value is assigned a lower index, and make use of Theorem 4.5 and part (a) of Theorem 4.6, which continue to hold when $\boldsymbol{N}-\boldsymbol{N}_{0}$ is replaced with $\boldsymbol{N}-\frac{1}{K} \boldsymbol{N 1 1 ^ { \prime }}$. The next theorem provides the requisite adaptation of part (b) of Theorem 4.6 to the present case.

Theorem 4.17. Let the transition matrix $N$ describing movement of $n$ individuals across $K$ ordinal states have the expansion (18). Then the only measure of imperfection in individual mobility lying between -1 and 1 that satisfies the IIR, NOM and ERI properties, and also satisfies the LH, DR, PIN and TI properties in the special case of multiples of EIM matrices, is

$$
M_{I S}=\frac{2}{n(K-1)} \sum_{i=1}^{K}\left(\frac{K+1}{2}-i\right) n_{\cdot i}=-\frac{2}{n(K-1)} \sum_{i=1}^{K} \sum_{j=1}^{K-1} \eta_{i j} .
$$

Further the extreme values of the measure, -1 and 1 , occur when $n_{\cdot 1}=n_{\cdot K}$ and $n$, respectively.

The signed measure $M_{I S}$ is insensitive to PEMs. In fact, it can be shown to be a function of $\boldsymbol{N}_{S}$ given by (20), which is the structural part of the difference matrix $\boldsymbol{N}-\frac{1}{K} \boldsymbol{N} \mathbf{1 1}{ }^{\prime}$. In order to measure the exchange part, we have to expand $\boldsymbol{N}_{E}$ given by (21) as in (6). The measure developed in Section 4.5 for linear combinations of PEMs, and characterized in Theorem 4.13, will be applicable. In fact, Theorem 4.14 also continues to hold when the matrix $\boldsymbol{N}_{2}$ is replaced by $\boldsymbol{N}_{E}$. For this purpose, the $\alpha_{i j}$ s defined in Theorem 2.3 have to be modified by replacing $\boldsymbol{N}-\boldsymbol{N}_{0}-\boldsymbol{N}_{1}\left(\right.$ or $\boldsymbol{N}_{2}$ ) with $\boldsymbol{N}_{E}$, as follows.

$\alpha_{i j}=-\sum_{k=1}^{i} \sum_{l=1}^{j} \boldsymbol{e}_{k}^{\prime} \boldsymbol{N}_{E} \boldsymbol{e}_{l}, \quad k=1, \ldots, K-i, l=1, \ldots, K-j, i, j=1, \ldots, K$

It transpires that the requisite measure of exchange mobility accounting for imperfection in mobility is a scaled version of the sum of the $\alpha_{i j} \mathrm{~s}$, as described below.

Theorem 4.18. For a transition matrix $N$ describing movement of $n$ individuals across $K$ ordinal states, let $\boldsymbol{N}_{E}$ defined in (21) have the expan- 
sion $\boldsymbol{N}_{E}=\sum_{i=1}^{K-1} \sum_{j=1}^{K-1} \alpha_{i j}$, where the $\alpha_{i j}$ s are as in (41). Then the only measure of exchange mobility that accounts for imperfection in mobility and lies between -1 and 1 while satisfying the IIR, NOM and ERI properties, and also satisfying the LH, DR, PIN and TI properties in the special case of multiples of elemental PEM matrices, is

$$
\begin{aligned}
M_{I E} & =-\frac{4}{n(K-1)^{2}} \sum_{i=1}^{K} \sum_{j=1}^{K}\left(i-\frac{K+1}{2}\right)\left(j-\frac{K+1}{2}\right) n_{i j} \\
& =\frac{4}{n(K-1)^{2}} \sum_{i=1}^{K-1} \sum_{j=1}^{K-1} \alpha_{i j} .
\end{aligned}
$$

Further, the extreme values of the measure, -1 and 1 , occur when $n_{11}=$ $n_{K K}=\frac{n}{2}$ and $n_{1 K}=n_{K 1}=\frac{n}{2}$, respectively.

Example 4.4. Consider the inter-generational mobility data of Table 2.1. The values of $\alpha_{i j}$ defined in (41) are as follows.

$$
\left(\begin{array}{ccc}
\alpha_{11} & \cdots & \alpha_{16} \\
\vdots & \ddots & \vdots \\
\alpha_{61} & \cdots & \alpha_{66}
\end{array}\right)=\left(\begin{array}{rrrrrr}
-88.2 & -137.4 & -169.2 & -164.6 & -31.4 & -5.6 \\
-139.4 & -255.9 & -324.5 & -322.2 & -65.8 & -13.3 \\
-158.8 & -309.6 & -418.2 & -438.4 & -122.9 & -30.8 \\
-152.5 & -297.9 & -414.7 & -476.7 & -177.5 & -62.0 \\
-7.4 & -21.8 & -61.1 & -98.2 & -164.5 & -77.9 \\
1.4 & -2.3 & -19.3 & -36.8 & -83.9 & -61.5
\end{array}\right) .
$$

The measure $M_{I}$ of imperfection in individual mobility works out to be 0.7188 .

Turning to the signed measure $M_{I S}$ of imperfection in individual mobility (which captures the structural part only), its value is found to be exactly the same, indicating departure from perfect mobility only in the direction of upward movement. It may be recalled that perfect mobility serves as the point of reference here. If the immobile situation is chosen as reference, the direction of structural mobility reverses (see Example 4.2).

The value of $M_{I E}$, the signed measure of the exchange part of imperfection in mobility, is -0.1719 . This is found to be in the direction of concordance between occupational status across generations. It transpires that for this data set the direction of measured exchange mobility also depends on the point of reference. When the immobile situation had been chosen as reference, net exchange mobility had been found to be in the direction of discordance (see Example 4.3).

It is also seen from the above comparisons that the magnitudes of all the measures are higher when perfect mobility is used as reference. Thus, perfect mobility is a very distant reference for the actual status of all the aspects of mobility in the present case. 


\section{Measures for nominal data}

\subsection{Alternative expansions and axiom}

In the case of ordinal data, we had defined some elemental matrices to describe individual, structural and exchange movements. The negative versions of the matrices represented movements in the opposite direction. However, for nominal data, opposite movements have to be considered separately. For this purpose, we redefine some earlier expansions in terms of an extended class of elemental matrices.

To begin with, we express (3) as

$$
\boldsymbol{N}-\boldsymbol{N}_{0}=\sum_{i=1}^{K} \sum_{j=1}^{K} \sum_{l=1}^{K} \tau_{i j l} \boldsymbol{K}_{i j, l-j},
$$

where, for $i, j, l=1, \ldots, K, \boldsymbol{K}_{i j, l-j}=-\boldsymbol{e}_{i} \boldsymbol{e}_{j}^{\prime}+\boldsymbol{e}_{i} \boldsymbol{e}_{l}^{\prime}=-\boldsymbol{K}_{i l, j-l}$, and

$$
\tau_{i j l}= \begin{cases}\xi_{i j, l-j} & \text { if } l>j, \xi_{i j, l-j}>0 \\ -\xi_{i l, j-l} & \text { if } l<j, \xi_{i l, j-l}<0 \\ 0 & \text { otherwise }\end{cases}
$$

Here, the definition of the $\boldsymbol{K}_{i j k} \mathrm{~s}$ is extended to the case of negative third suffix, so that the set of elemental EIM matrices now cover all possible swaps of destination states of individuals from every given source state. In the new expansion of $\boldsymbol{N}-\boldsymbol{N}_{0}$ all the $\tau_{i j l}$ s are non-negative. Further $\tau_{i j j}=0$, and at least one of $\tau_{i j l}$ and $\tau_{i l j}$ is zero. For every expansion of the form (43) with nonnegative coefficients one can obtain a unique expansion (3) by choosing $\xi_{i j k}=\tau_{i j, j+k}-\tau_{i, j+k, j}, k=1, \ldots, K-j, j=1, \ldots, K-1, i=1, \ldots, K$.

Likewise, we express (14) as

$$
\boldsymbol{N}_{1}=\sum_{j=1}^{K} \sum_{i=1}^{K} \pi_{j i} \boldsymbol{L}_{j, i-j}
$$

where, for $i, j=1, \ldots, K, \boldsymbol{L}_{j, i-j}=-\mathbf{1} \boldsymbol{e}_{j}^{\prime}+\mathbf{1} \boldsymbol{e}_{i}^{\prime}=-\boldsymbol{L}_{i, j-i}$ and

$$
\pi_{j i}= \begin{cases}\phi_{j, i-j} & \text { if } i>j, \phi_{j, i-j}>0 \\ -\phi_{i, j-i} & \text { if } i<j, \phi_{i, j-i}<0 \\ 0 & \text { otherwise. }\end{cases}
$$

The new definition of $\boldsymbol{L}_{j, i-j}$ is no longer restricted to positive values of the second suffix. In the new expansion of $\boldsymbol{N}_{1}$ all coefficients are positive. Further $\pi_{i i}=0$ and at least one of $\pi_{i j}$ and $\pi_{j i}$ is zero. For every expansion of the form (44) with nonnegative coefficients one can obtain a 
unique expansion (14) by choosing $\phi_{j k}=\pi_{j, j+k}-\pi_{j+k, j}, k=1, \ldots, K-j$, $j=1, \ldots, K-1$.

We also express (31) as

$$
\boldsymbol{N}_{2}=\sum_{i=1}^{K-1} \sum_{k=1}^{K-i} \sum_{j=1}^{K} \sum_{l=1}^{K} \gamma_{i j k l} \boldsymbol{F}_{i j k, l-j} .
$$

where, for $k=1, \ldots, K-i, i=1, \ldots, K-1$ and $j, l=1, \ldots, K$,

$$
\begin{aligned}
\boldsymbol{F}_{i j k, l-j} & =-\boldsymbol{e}_{i} \boldsymbol{e}_{j}^{\prime}+\boldsymbol{e}_{i+k} \boldsymbol{e}_{j}^{\prime}+\boldsymbol{e}_{i} \boldsymbol{e}_{l}^{\prime}-\boldsymbol{e}_{i+k} \boldsymbol{e}_{l}^{\prime}=-\boldsymbol{F}_{i l k, j-l}, \\
\gamma_{i j k l} & = \begin{cases}\beta_{i j k, l-j} & \text { if } l>j, \beta_{i j k, l-j}>0, \\
-\beta_{i l k, j-l} & \text { if } l<j, \beta_{i l k, j-l}<0, \\
0 & \text { otherwise. }\end{cases}
\end{aligned}
$$

The new definition of $\boldsymbol{F}_{i j k, l-j}$ permits negative values of the fourth suffix. The $\gamma_{i j k l} \mathrm{~s}$ satisfy the conditions

$$
\gamma_{i j k l} \geq 0, \quad \gamma_{i j k j}=0, \quad \gamma_{i j k l} \gamma_{i l k j}=0,
$$

for $k=1, \ldots, K-i, i=1, \ldots, K-1, j, l=1, \ldots, K$. The expansion (31) can be retrieved uniquely from (45) by choosing

$\beta_{i j k l}=\gamma_{i j k, j+l}-\gamma_{i, j+l, k, j}, \quad k=1, \ldots, K-i, l=1, \ldots, K-j, i, j=1, \ldots, K-1$.

For nominal data, the property LH is relevant, but PIN and TI are not. Instead, we need to have equal weights for all elemental movements, so that movements between all pairs of states are treated in the same manner. This property may be formalized as follows.

Uniformity of movements (UM). If $M$ is a non-zero matrix representing an elemental movement (individual, structural or exchange), the measure $m$ satisfies $m(\boldsymbol{M})=c$ for some positive constant $c$. In particular,

(a) For $i, j, l=1, \ldots, K, l \neq j$ and some positive constant $c$,

$$
m\left(\boldsymbol{K}_{i j, l-j}\right)= \begin{cases}c & \text { if } l \neq j, \\ 0 & \text { if } l=j,\end{cases}
$$

(b) For $i, j=1, \ldots, K, i \neq j$ and some positive constant $c$,

$$
m\left(\boldsymbol{L}_{j, i-j}\right)= \begin{cases}c & \text { if } i \neq j \\ 0 & \text { if } i=j\end{cases}
$$

(c) For $k=1, \ldots, K-i, \quad i=1, \ldots, K-1, \quad j, l=1, \ldots, K$ and some positive constant $c$,

$$
m\left(\boldsymbol{F}_{i j k, l-j}\right)= \begin{cases}c & \text { if } l \neq j, \\ 0 & \text { if } l=j .\end{cases}
$$




\subsection{Measure for individual mobility}

It is obvious that for a measure $m$ of multiples of EIM matrices satisfying $\mathrm{LH}$ and UM, $m\left(a \boldsymbol{K}_{i j, l-j}\right) \propto|a|$ for $i, l, j=1, \ldots, K$ and any real $a$. The next theorem extends the measure to linear combinations of EIM matrices such as those used in (43).

Theorem 5.1. A measure of mobility of a combination of EIMs represented by the weighted sum $\sum_{i=1}^{K} \sum_{j=1}^{K} \sum_{l=1}^{K} \tau_{i j l} \boldsymbol{K}_{i j, l-j}$ satisfies the IIR, NOM and ERI properties, and also satisfies the LH and UM properties in the special case of multiples of EIM matrices, if and only if it is proportional to $\sum_{i=1}^{K} \sum_{j=1}^{K} \sum_{l=1}^{K} \tau_{i j l}$.

Multiple expansions of $\boldsymbol{N}-\boldsymbol{N}_{0}$ of the form (43) would have possibly different measures as per Theorem 5.1. In the next theorem we resolve this ambiguity by using the MIN property as in Section 4.2, and use appropriate scaling.

Theorem 5.2. For a transition matrix $N$ describing movement of $n$ individuals across $K$ nominal states, the only measure of overall individual mobility lying between 0 and 1 that satisfies MIN, in which the measure of linear combinations of EIMs satisfies the IIR, NOM and ERI properties, and also satisfies the LH and UM properties in the special case of multiples of EIM matrices, is the Prais-Bibby measure (Prais, 1955; Bibby, 1975)

$$
M_{N}=1-\frac{1}{n} \sum_{i=1}^{K} n_{i i} .
$$

Further, the lower bound 0 is achieved by an immobile matrix, and the upper bound 1 is achieved by any transition matrix with zero diagonal elements.

Example 5.1. For the inter-generational mobility data of Table 2.1, the measure $M_{N}$ of individual mobility turns out to be 0.6488 . In contrast, if the Bartholomew measure had been scaled to the interval [0,1], it would have been only 0.1764 . The latter scale is calibrated to have the largest value only when all individuals have the largest possible jump. As a result, smaller jumps on the part of most individuals look very small in comparison. The value of $M_{S}$ is even smaller (-0.0179), because of cancelation of upward and downward movements. The measure $M_{N}$ captures the number of direct jumps (irrespective of size) which, for this data set, is almost two-thirds of the maximum possible value.

\subsection{Measure of structural mobility}

A measure $m$ of multiples of elemental SCM matrices satisfying LH and UM is easily seen to have the form $m\left(a \boldsymbol{L}_{j, l-j}\right) \propto|a|$ for $l, j=1, \ldots, K$ and any 
real $a$. For extension to linear combinations of elemental SCM matrices such as those used in (44), we present the next theorem.

Theorem 5.3. An index of structural mobility of a combination of movements represented by the weighted sum $\sum_{j=1}^{K} \sum_{l=1}^{K} \pi_{j l} \boldsymbol{L}_{j, l-j}$ satisfies the IIR, NOM and ERI properties, and also satisfies the LH and UM properties in the special case of multiples of elemental SCM matrices, if and only if it is proportional to $\sum_{j=1}^{K} \sum_{l=1}^{K} \pi_{j l}$.

The ambiguity of different measures of multiple representations is resolved by using the MIN property, and the intended measure with appropriate scaling is characterized through the next theorem.

Theorem 5.4. For a transition matrix $N$ describing movement of $n$ individuals across $K$ nominal states, the only measure of overall structural mobility lying between 0 and 1 that satisfies MIN, in which the measure of linear combinations of elemental SCMs satisfies the IIR, NOM and ERI properties, and also satisfies the LH and UM properties in the special case of multiples of elemental SCM matrices, is

$$
\boldsymbol{M}_{S N}=\frac{1}{2 n} \sum_{i=1}^{K}\left|n_{\cdot i}-n_{i \cdot}\right| .
$$

Further, the lower bound is attained when the marginal counts before and after transition are identical, while the upper bound it attained when all individuals belong to a single state before transition and they all transit to a different but common state.

Example 5.2. For the inter-generational mobility data of Table 2.1, the measure $M_{S N}$ of structural ability happens to be 0.0518 . It may be recalled that when the ranks of the occupations are taken into considerations, the measure $M_{S}$ of structural mobility is -0.0179 (see Example 4.1). The larger value of $M_{S N}$ compared to $M_{S}$ is because of the fact that the range of $M_{S}$ is not scaled by different extremes of the ordered states. The value of $M_{S N}$ may also be contrasted with the value of $M_{N}$, which is 0.6488 (see Example 5.1). The value of $M_{S N}$ is only a small fraction of the latter. This fact indicates that much of the individual movements happen in a way that does not contribute to structural mobility.

\subsection{Measure of exchange mobility}

Clearly a measure $m$ of multiples of elemental PEM matrices satisfying LH and UM has the form $m\left(a \boldsymbol{F}_{i j k l}\right) \propto|a|$ for $k=1, \ldots, K-i, l=1, \ldots, K-j$, $i, j=1, \ldots, K-1$ and any real $a$. The next theorem provides extension to combinations of elemental PEM matrices such as those used in (45). 
Theorem 5.5. A measure of exchange mobility $m$ for combinations of PEM matrices satisfies the IIR, NOM and ERI properties, and also satisfies the LH and UM properties in the special case of multiples of elemental PEM matrices, if an only if

$$
m\left(\sum_{i=1}^{K-1} \sum_{j=1}^{K-1} \sum_{k=1}^{K-i} \sum_{l=1}^{K-j} \beta_{i j k l} \boldsymbol{F}_{i j k l}\right) \propto \sum_{i=1}^{K-1} \sum_{j=1}^{K-1} \sum_{k=1}^{K-i} \sum_{l=1}^{K-j}\left|\beta_{i j k l}\right| .
$$

Usage of the MIN property and scaling characterizes the requisite measure as follows.

Theorem 5.6. For a transition matrix $\boldsymbol{N}$ describing movement of $n$ individuals across $K$ nominal states, the measure of exchange mobility

$$
\begin{array}{r}
M_{E N}=\frac{K}{n(K-1)} \min \left\{\sum_{i=1}^{K-1} \sum_{j=1}^{K-1} \sum_{k=1}^{K-i} \sum_{l=1}^{K-j}\left|\beta_{i j k l}\right|:\right. \\
\left.\boldsymbol{N}_{2}=\sum_{i=1}^{K-1} \sum_{j=1}^{K-1} \sum_{k=1}^{K-i} \sum_{l=1}^{K-j} \beta_{i j k l} \boldsymbol{F}_{i j k l}\right\},
\end{array}
$$

where the minimum is over all possible representations of $\boldsymbol{N}_{2}=(\boldsymbol{I}-$ $\left.\frac{1}{K} \mathbf{1 1}^{\prime}\right)\left(\boldsymbol{N}-\boldsymbol{N}_{0}\right)$ of the form (31), is the only measure lying between 0 and 1 that satisfies MIN, in which the measure of exchange mobility of the individual representations satisfies the IIR, NOM and ERI properties, and also satisfies the LH and UM properties in the special case of multiples of elemental PEM matrices. The smallest value, 0 , is attained if and only if $\boldsymbol{N}_{2}=\mathbf{0}$. The largest value, 1 , is achieved when

$$
\boldsymbol{N}=\left(\begin{array}{ccccc}
0 & 0 & \cdots & 0 & n \\
0 & 0 & \cdots & 0 & 0 \\
\vdots & \vdots & \ddots & \vdots & \vdots \\
0 & 0 & \cdots & 0 & 0 \\
0 & 0 & \cdots & 0 & 0
\end{array}\right) \quad \text { or } \boldsymbol{N}=\left(\begin{array}{ccccc}
0 & n / K & 0 & \cdots & 0 \\
0 & 0 & n / K & \cdots & 0 \\
0 & 0 & 0 & \ddots & \vdots \\
\vdots & \vdots & \vdots & \ddots & n / K \\
n / K & 0 & 0 & \cdots & 0
\end{array}\right)
$$

for some ordering of the states.

Computation of $M_{E N}$ is a non-trivial issue. One can write the constraint in $(48)$ as

$$
\operatorname{vec}\left(\boldsymbol{N}_{2}\right)=\sum_{i=1}^{K-1} \sum_{j=1}^{K-1} \sum_{k=1}^{K-i} \sum_{l=1}^{K-j} \beta_{i j k l} \operatorname{vec}\left(\boldsymbol{F}_{i j k l}\right),
$$

where $\operatorname{vec}(\cdot)$ indicates the long vector obtained by concatenating successive columns of a matrix. A more compact representation of the above equation is

$$
\boldsymbol{A} \boldsymbol{\beta}=\operatorname{vec}\left(\boldsymbol{N}_{2}\right)
$$


where $\boldsymbol{\beta}$ is the vector of $\beta_{i j k l} \mathrm{~s}$ arranged in any particular order, and $\boldsymbol{A}$ is the matrix obtained by stacking the vectors $\operatorname{vec}\left(\boldsymbol{F}_{i j k l}\right)$ in the same order. It may be noted that the matrix $\boldsymbol{A}$ has order $K^{2} \times K^{2}(K-1)^{2} / 4$, but its rank is only $(K-1)^{2}$, the latter being the dimension of the linear space of the matrices of the form $\boldsymbol{F}_{i j k l}$. Since the above is a consistent and over-determined set of linear equations, it has infinitely many solutions. A particular solution is

$$
\boldsymbol{\beta}_{0}=\boldsymbol{A}^{+} \operatorname{vec}\left(\boldsymbol{N}_{2}\right),
$$

where $\boldsymbol{A}^{+}$is the Moore-Penrose pseudo inverse of $\boldsymbol{A}$, while the general solution can be expressed as

$$
\boldsymbol{\beta}=\boldsymbol{\beta}_{0}-\boldsymbol{X} \boldsymbol{\alpha},
$$

where $\boldsymbol{X}$ is a matrix whose columns constitute a basis of the null space of the matrix $\boldsymbol{A}$, and $\boldsymbol{\alpha}$ is a completely arbitrary vector. By comparing the number of columns of $\boldsymbol{A}$ and the rank of $\boldsymbol{A}$, it can be concluded that $\boldsymbol{X}$ is a $K^{2}(K-1)^{2} / 4 \times\left(K^{2} / 4-1\right)(K-1)^{2}$ matrix having full column rank. It follows from the above discussion and (48) that

$$
M_{E N}=\frac{K}{n(K-1)} \min _{\boldsymbol{\alpha}}\left\|\boldsymbol{\beta}_{0}-\boldsymbol{X} \boldsymbol{\alpha}\right\|,
$$

where $\|\cdot\|$ represents the $L_{1}$ norm, i.e., the sum of absolute values of the elements of the vector. The minimum value on the right hand side of the above equation is the $L_{1}$ norm of the residual vector of the $L_{1}$ regression, also known as the least absolute deviation (LAD) regression, of $\boldsymbol{\beta}_{0}$ on the columns of $\boldsymbol{X}$, with no intercept term. Even if the LAD estimator of $\alpha$ is not unique, the minimized value is unique. This quantity can be calculated by using freely available software, such as the lad function in the L1pack package of $\mathrm{R}$. An R program to compute the measure $M_{E N}$, and another measure $M_{I E N}$ defined in Theorem 5.9 below, is available from the authors on request.

Example 5.3. If the inter-generational mobility data of Table 2.1 is regarded as nominal, the measure $M_{E N}$ of exchange mobility happens to be 0.3995. Once again, in contrast with ordinal data (see Example 4.3, where $M_{E}$ was found to be 0.0497), exchange mobility measures larger when the states are regarded as nominal.

\subsection{Measures of imperfection in mobility}

We now replace the matrix $\boldsymbol{N}-\boldsymbol{N}_{0}$ with $\boldsymbol{N}-\frac{1}{K} \boldsymbol{N} \mathbf{1 1}{ }^{\prime}$, as we did in Section 4.6, and follow the development of Section 5.2. We can actually make use of the measure of combinations of EIMs characterized axiomatically in Theorem 5.1. The different representations of $\boldsymbol{N}-\frac{1}{K} \boldsymbol{N 1 1 ^ { \prime }}$ of the form (38) 
would lead to different values of this measure. Therefore, by relying on the property MIN, we adapt Theorem 5.2 to arrive at a unique measure as follows.

Theorem 5.7. For a transition matrix $N$ describing movement of $n$ individuals across $K$ nominal states, the only measure of imperfection in mobility that lies between 0 and 1 and satisfies MIN, in which the measure of linear combinations of EIMs satisfies the IIR, NOM and ERI properties, and also satisfies the LH and UM properties in the special case of multiples of EIM matrices, is

$$
M_{I N}=\frac{1}{n} \sum_{i=1}^{K} \sum_{j=1}^{K}\left|n_{i j}-\frac{n_{i \cdot}}{K}\right| .
$$

Further, the lower bound 0 is achieved by a transition matrix with perfect mobility, and the upper bound 1 is achieved by a transition matrix having the counts in every row split evenly between 0 and another constant.

For measuring the structural component of imperfection in mobility, we can use a representation of (20) similar to (44), and the measure of combinations of SCMs characterized in Theorem 5.3. As different representations would produce different values of this measure, we can use the property MIN to resolve the ambiguity and characterize the requisite measure in the following adaptation of Theorem 5.4.

Theorem 5.8. For a transition matrix $\boldsymbol{N}$ describing movement of $n$ individuals across $K$ nominal states, the only measure of structural imperfection in mobility that lies between 0 and 1 and satisfies MIN, in which the measure of linear combinations of SCMs satisfies the IIR, NOM and ERI properties, and also satisfies the LH and UM properties in the special case of multiples of elemental SCM matrices, is

$$
M_{I S N}=\frac{K}{2 n(K-1)} \sum_{i=1}^{K}\left|n_{\cdot i}-\frac{n}{K}\right| .
$$

Further, the measure is equal to 0 for a transition matrix with equal column sums, and is equal to 1 for a transition matrix having zero elements everywhere except in any one column.

Turning to pure exchange movements associated with imperfection in mobility, we can express the matrix $\boldsymbol{N}_{E}$ in (21) as a combination of elemental PEMs such as (31), for which a measure had been characterized in Theorem 5.5. After accounting for multiple representations through the MIN property, we obtain the scaled measure given in the next theorem. 
Theorem 5.9. For a transition matrix $\boldsymbol{N}$ describing movement of $n$ individuals across $K$ nominal states, the measure of pure exchange movements accounting for imperfection in mobility

$$
\begin{array}{r}
M_{I E N}=\frac{K^{2}}{n(K-1)^{2}} \min \left\{\sum_{i=1}^{K-1} \sum_{j=1}^{K-1} \sum_{k=1}^{K-i} \sum_{l=1}^{K-j}\left|\beta_{i j k l}\right|:\right. \\
\left.\boldsymbol{N}_{E}=\sum_{i=1}^{K-1} \sum_{j=1}^{K-1} \sum_{k=1}^{K-i} \sum_{l=1}^{K-j} \beta_{i j k l} \boldsymbol{F}_{i j k l}\right\},
\end{array}
$$

where the minimum is over all possible representations of $\boldsymbol{N}_{E}=(\boldsymbol{I}-$ $\left.\frac{1}{K} \mathbf{1 1}^{\prime}\right) \boldsymbol{N}\left(\boldsymbol{I}-\frac{1}{K} \mathbf{1 1}^{\prime}\right)$ of the form(31), is the only measure lying between 0 and 1 that satisfies MIN, in which the measure of linear combinations of PEMs satisfies the IIR, NOM and ERI properties, and also satisfies the $\mathrm{LH}$ and UM properties in the special case of multiples of elemental PEM matrices. Further, the measure is equal to 0 for a transition matrix with all elements equal to $n / K^{2}$, and is equal to 1 for a transition matrix having zero elements everywhere except at a single non-diagonal position.

Computation of the measure $M_{I E N}$ for any given transition matrix can be made in the manner described in the end of Section 5.4.

Example 5.4. Regarding the inter-generational mobility data of Table 2.1 as nominal, the measure $M_{I N}$ of individual mobility is computed as 0.7326 , the measure $M_{I S N}$ of structural mobility is found to be 0.3412 and the measure $M_{I E N}$ of exchange mobility turns out to be 0.2159 . These values represent the extents of individual, structural and exchange movements, respectively, seen as departure from perfect mobility. When the immobile situation had been used as reference, these numbers were 0.6488, 0.0518 and 0.3995 , respectively. While the two sets of numbers are not comparable, one can broadly conclude that the transition matrix is structurally far from perfect mobility, but not very far from the immobile situation.

\section{Concluding remarks}

A summary of the various measures of extent of mobility and imperfection in mobility, derived in this paper is given in Table 6.1.

A key aspect of the decompositions presented in this paper is the use of a special transition matrix as reference. Here we have considered the cases of perfect immobility and perfect mobility for this purpose. Similar decompositions, ordering, and measures can be developed around other reference matrices. A case in point is that of independence of source and destination states with arbitrary destination distribution, which has been referred to by Sobel (1983) as 'perfect mobility'. 


\begin{tabular}{|c|c|c|c|c|c|c|c|}
\hline $\begin{array}{l}\text { Name of } \\
\text { measure }\end{array}$ & $\begin{array}{l}\text { Defined } \\
\text { in }\end{array}$ & $\begin{array}{l}\text { Aspect of } \\
\text { mobility }\end{array}$ & $\begin{array}{l}\text { Type of } \\
\text { mobility }\end{array}$ & $\begin{array}{l}\text { Nature of } \\
\text { states }\end{array}$ & $\begin{array}{l}\text { Directional } \\
\text { property }\end{array}$ & $\begin{array}{l}\text { Range of } \\
\text { values }\end{array}$ & $\begin{array}{l}\text { Characterizing } \\
\text { properties }\end{array}$ \\
\hline$M_{B}$ & $(23)$ & Extent & Individual & $\begin{array}{l}\text { Ordinal, } \\
\text { equispaced }\end{array}$ & $\begin{array}{l}\text { Direction- } \\
\text { free }\end{array}$ & {$[0, K-1]$} & $\begin{array}{l}\text { MIN, IIR, NOM, } \\
\text { ERI, SYM, PIN, } \\
\text { TI, LH }\end{array}$ \\
\hline$M_{S}$ & (24) & Extent & Individual & $\begin{array}{l}\text { Ordinal, } \\
\text { equispaced }\end{array}$ & Directional & {$[-1,1]$} & $\begin{array}{l}\text { IIR, NOM, ERI, } \\
\text { DR, PIN, TI, LH }\end{array}$ \\
\hline$M_{S}$ & (28) & Extent & Structural & $\begin{array}{l}\text { Ordinal, } \\
\text { equispaced }\end{array}$ & Directional & {$[-1,1]$} & $\begin{array}{l}\text { IIR, NOM, ERI, } \\
\text { DR, PIN, TI, LH }\end{array}$ \\
\hline$M_{E}$ & (35) & Extent & Exchange & $\begin{array}{l}\text { Ordinal, } \\
\text { equispaced }\end{array}$ & Directional & {$\left[-\frac{1}{9}, \frac{8}{9}\right]$} & $\begin{array}{l}\text { IIR, NOM, ERI, } \\
\text { DR, PIN, TI, LH }\end{array}$ \\
\hline$M_{N}$ & $(46)$ & Extent & Individual & Nominal & $\begin{array}{l}\text { Direction- } \\
\text { free }\end{array}$ & {$[0,1]$} & $\begin{array}{l}\text { MIN, IIR, NOM, } \\
\text { ERI, UM, LH }\end{array}$ \\
\hline$M_{S N}$ & (47) & Extent & Structural & Nominal & $\begin{array}{l}\text { Direction- } \\
\text { free }\end{array}$ & {$[0,1]$} & $\begin{array}{l}\text { MIN, IIR, NOM, } \\
\text { ERI, UM, LH }\end{array}$ \\
\hline$M_{E N}$ & (48) & Extent & Exchange & Nominal & $\begin{array}{l}\text { Direction- } \\
\text { free }\end{array}$ & {$[0,1]$} & $\begin{array}{l}\text { MIN, IIR, NOM, } \\
\text { ERI, UM, LH }\end{array}$ \\
\hline$M_{I}$ & (39) & $\begin{array}{l}\text { Imper- } \\
\text { fection }\end{array}$ & Individual & $\begin{array}{l}\text { Ordinal, } \\
\text { equispaced }\end{array}$ & $\begin{array}{l}\text { Direction- } \\
\text { free }\end{array}$ & {$[0,1]$} & $\begin{array}{l}\text { MIN, IIR, NOM, } \\
\text { ERI, SYM, PIN, } \\
\text { TI, LH }\end{array}$ \\
\hline$M_{I S}$ & (40) & $\begin{array}{l}\text { Imper- } \\
\text { fection }\end{array}$ & Individual & $\begin{array}{l}\text { Ordinal, } \\
\text { equispaced }\end{array}$ & Directional & {$[-1,1]$} & $\begin{array}{l}\text { IIR, NOM, ERI, } \\
\text { DR, PIN, TI, LH }\end{array}$ \\
\hline$M_{I S}$ & (40) & $\begin{array}{l}\text { Imper- } \\
\text { fection }\end{array}$ & Structural & $\begin{array}{l}\text { Ordinal, } \\
\text { equispaced }\end{array}$ & Directional & {$[-1,1]$} & $\begin{array}{l}\text { IIR, NOM, ERI, } \\
\text { DR, PIN, TI, LH }\end{array}$ \\
\hline$M_{I E}$ & $(42)$ & $\begin{array}{l}\text { Imper- } \\
\text { fection }\end{array}$ & Exchange & $\begin{array}{l}\text { Ordinal, } \\
\text { equispaced }\end{array}$ & Directional & {$[-1,1]$} & $\begin{array}{l}\text { IIR, NOM, ERI, } \\
\text { DR, PIN, TI, LH }\end{array}$ \\
\hline$M_{I N}$ & (49) & $\begin{array}{l}\text { Imper- } \\
\text { fection }\end{array}$ & Individual & Nominal & $\begin{array}{l}\text { Direction- } \\
\text { free }\end{array}$ & {$[0,1]$} & $\begin{array}{l}\text { MIN, IIR, NOM, } \\
\text { ERI, UM, LH }\end{array}$ \\
\hline$M_{I S N}$ & (50) & $\begin{array}{l}\text { Imper- } \\
\text { fection }\end{array}$ & Structural & Nominal & $\begin{array}{l}\text { Direction- } \\
\text { free }\end{array}$ & {$[0,1]$} & $\begin{array}{l}\text { MIN, IIR, NOM, } \\
\text { ERI, UM, LH }\end{array}$ \\
\hline$M_{I E N}$ & (51) & $\begin{array}{l}\text { Imper- } \\
\text { fection }\end{array}$ & Exchange & Nominal & $\begin{array}{l}\text { Direction- } \\
\text { free }\end{array}$ & {$[0,1]$} & $\begin{array}{l}\text { MIN, IIR, NOM, } \\
\text { ERI, UM, LH }\end{array}$ \\
\hline
\end{tabular}

Table 6.1: Summary of various measures of extent of mobility and imperfection in mobility, derived axiomatically in this paper 
The approach used here for ordinal data with equidistant states can be generalized to the case of non-equidistant states as well. This may be done by assigning suitable weights to the categories, for use in the sums of $\eta_{i j} \mathrm{~s}$, $\theta_{j}$ s and $\alpha_{i j}$ s. The resulting measures have to be scaled afresh.

\section{Appendix: Proofs of theorems}

Here we have used the notations $\mathbf{1}$ and $\mathbf{0}$ for vectors of size $K$ having all elements equal to 1 and all elements equal to 0 , respectively, and the notations $\boldsymbol{e}_{1}, \boldsymbol{e}_{2}, \ldots, \boldsymbol{e}_{K}$ for the successive columns of the $K \times K$ identity matrix.

Proof of Theorem 2.1. We shall first show that if the claimed expansion is indeed possible, then the $\eta_{i j}$ s must have the given expressions. It follows from the expansion that for any $i=1,2, \ldots, K$ and $j=1,2, \ldots, K-1$,

$$
\begin{aligned}
\boldsymbol{e}_{i}^{\prime}(\boldsymbol{N} & \left.-\boldsymbol{N}_{0}\right) \boldsymbol{e}_{j} \\
& =\boldsymbol{e}_{i}^{\prime}\left(\sum_{k=1}^{K} \sum_{l=1}^{K-1} \eta_{k l} \boldsymbol{G}_{k l}\right) \boldsymbol{e}_{j}=\sum_{l=1}^{K-1}\left(\eta_{i l} \boldsymbol{e}_{l+1}^{\prime}-\eta_{i l} \boldsymbol{e}_{l}^{\prime}\right) \boldsymbol{e}_{j} \\
& =\sum_{l=1}^{K-1} \eta_{i l} \boldsymbol{e}_{l+1}^{\prime} \boldsymbol{e}_{j}-\sum_{l=1}^{K-1} \eta_{i l} \boldsymbol{e}_{l}^{\prime} \boldsymbol{e}_{j}= \begin{cases}-\eta_{i 1} & \text { if } j=1, \\
\eta_{i, j-1}-\eta_{i j} & \text { if } j=2,3, \ldots, K-1 .\end{cases}
\end{aligned}
$$

These equations are equivalent to the given expressions of the $\eta_{i j} \mathrm{~s}$.

Having proved the uniqueness of the stated expansion subject to its existence, we establish the existence by showing that

$$
\boldsymbol{e}_{i}^{\prime}\left(\boldsymbol{N}-\boldsymbol{N}_{0}\right) \boldsymbol{e}_{j}=\boldsymbol{e}_{i}^{\prime}\left(\sum_{k=1}^{K} \sum_{l=1}^{K-1} \eta_{k l} \boldsymbol{G}_{k l}\right) \boldsymbol{e}_{j} \quad \text { for } i, j=1,2, \ldots, K .
$$

We can reverse the above steps to show that this identity holds for $i=$ $1,2, \ldots, K$ and $j=1,2, \ldots, K-1$. Therefore we only need to show that it also holds for $i=1,2, \ldots, K$ and $j=K$. Indeed, for $i=1,2, \ldots, K$,

$$
\begin{aligned}
\boldsymbol{e}_{i}^{\prime}(\boldsymbol{N} & \left.-\boldsymbol{N}_{0}\right) \boldsymbol{e}_{K} \\
& =\boldsymbol{e}_{i}^{\prime}\left(\boldsymbol{N}-\boldsymbol{N}_{0}\right)\left(\mathbf{1}-\sum_{j=1}^{K-1} \boldsymbol{e}_{j}\right) \quad\left(\text { since row sums of } \boldsymbol{N}-\boldsymbol{N}_{0} \text { are } 0\right) \\
& =\boldsymbol{e}_{i}^{\prime}\left(\boldsymbol{N}-\boldsymbol{N}_{0}\right)\left(-\sum_{j=1}^{K-1} \boldsymbol{e}_{j}\right)=\boldsymbol{e}_{i}^{\prime}\left(\sum_{k=1}^{K} \sum_{l=1}^{K-1} \eta_{k l} \boldsymbol{G}_{k l}\right)\left(-\sum_{j=1}^{K-1} \boldsymbol{e}_{j}\right) \\
& =\boldsymbol{e}_{i}^{\prime}\left(\sum_{k=1}^{K} \sum_{l=1}^{K-1} \eta_{k l} \boldsymbol{G}_{k l}\right)\left(\mathbf{1}-\sum_{j=1}^{K-1} \boldsymbol{e}_{j}\right)=\boldsymbol{e}_{i}^{\prime}\left(\sum_{k=1}^{K} \sum_{l=1}^{K-1} \eta_{k l} \boldsymbol{G}_{k l}\right) \boldsymbol{e}_{K},
\end{aligned}
$$

where the penultimate step follows from the fact that each row sum of every $\boldsymbol{G}_{k l}$ is 0 . This completes the proof. 
Proof of Theorem 2.2. It follows from the given expansion of $M$ that for $j=1,2, \ldots, K-1$

$$
\begin{aligned}
\boldsymbol{e}_{1}^{\prime} \boldsymbol{M} \boldsymbol{e}_{j} & =\sum_{l=1}^{K-1} \sum_{k=1}^{K-1} \alpha_{l k}\left(-\boldsymbol{e}_{1}^{\prime} \boldsymbol{e}_{l} \boldsymbol{e}_{k}^{\prime} \boldsymbol{e}_{j}+\boldsymbol{e}_{1}^{\prime} \boldsymbol{e}_{l} \boldsymbol{e}_{k+1}^{\prime} \boldsymbol{e}_{j}+\boldsymbol{e}_{1}^{\prime} \boldsymbol{e}_{l+1} \boldsymbol{e}_{k}^{\prime} \boldsymbol{e}_{j}-\boldsymbol{e}_{1}^{\prime} \boldsymbol{e}_{l+1} \boldsymbol{e}_{k+1}^{\prime} \boldsymbol{e}_{j}\right) \\
& = \begin{cases}-\alpha_{11} & \text { if } j=1, \\
-\alpha_{1 j}+\alpha_{1, j-1} & \text { if } j=2, \ldots, K-1 .\end{cases}
\end{aligned}
$$

It also follows from the given expansion of $M$ that for $i=2, \ldots, K-1$ and $j=1,2, \ldots, K-1$

$$
\begin{aligned}
\boldsymbol{e}_{i}^{\prime} \boldsymbol{M} \boldsymbol{e}_{j} & =\sum_{l=1}^{K-1} \sum_{k=1}^{K-1} \alpha_{l k}\left(-\boldsymbol{e}_{i}^{\prime} \boldsymbol{e}_{l} \boldsymbol{e}_{k}^{\prime} \boldsymbol{e}_{j}+\boldsymbol{e}_{i}^{\prime} \boldsymbol{e}_{l} \boldsymbol{e}_{k+1}^{\prime} \boldsymbol{e}_{j}+\boldsymbol{e}_{i}^{\prime} \boldsymbol{e}_{l+1} \boldsymbol{e}_{k}^{\prime} \boldsymbol{e}_{j}-\boldsymbol{e}_{i}^{\prime} \boldsymbol{e}_{l+1} \boldsymbol{e}_{k+1}^{\prime} \boldsymbol{e}_{j}\right) \\
& = \begin{cases}-\alpha_{i 1}+\alpha_{i-1,1} & \text { if } j=1, \\
-\alpha_{i j}+\alpha_{i, j-1}+\alpha_{i-1, j}-\alpha_{i-1, j-1} & \text { if } j=2, \ldots, K-1,\end{cases}
\end{aligned}
$$

which are equivalent to the given expressions of the $\alpha_{i j}$ s. Therefore, if the given expansion exists, the $\alpha_{i j}$ s must have the stated expressions.

In order to prove the validity of the expansion, observe that the given expressions already satisfy the equations

$$
\boldsymbol{e}_{i}^{\prime} \boldsymbol{M} \boldsymbol{e}_{j}=\boldsymbol{e}_{i}^{\prime}\left(\sum_{l=1}^{K-1} \sum_{k=1}^{K-1} \alpha_{k l} \boldsymbol{E}_{k l}\right) \boldsymbol{e}_{j}
$$

for $1 \leq i, j \leq K-1$. We only have to establish that these equations also hold for $i=K, j=1, \ldots, K$ and $j=K, i=1, \ldots, K$. Indeed, we have

$$
\begin{aligned}
& \boldsymbol{e}_{K}^{\prime} \boldsymbol{M} \boldsymbol{e}_{j} \\
& =\left(\mathbf{1}-\sum_{i=1}^{K-1} \boldsymbol{e}_{i}\right)^{\prime} \boldsymbol{M} \boldsymbol{e}_{j}=-\sum_{i=1}^{K-1} \boldsymbol{e}_{i}^{\prime} \boldsymbol{M} \boldsymbol{e}_{j}=-\sum_{i=1}^{K-1} \boldsymbol{e}_{i}^{\prime}\left(\sum_{l=1}^{K-1} \sum_{k=1}^{K-1} \alpha_{k l} \boldsymbol{E}_{k l}\right) \boldsymbol{e}_{j} \\
& =\left(\mathbf{1}-\sum_{i=1}^{K-1} \boldsymbol{e}_{i}\right)^{\prime}\left(\sum_{l=1}^{K-1} \sum_{k=1}^{K-1} \alpha_{k l} \boldsymbol{E}_{k l}\right) \boldsymbol{e}_{j}=\boldsymbol{e}_{K}^{\prime}\left(\sum_{l=1}^{K-1} \sum_{k=1}^{K-1} \alpha_{k l} \boldsymbol{E}_{k l}\right) \boldsymbol{e}_{j}
\end{aligned}
$$

for $1 \leq j \leq K-1$, and

$$
\begin{aligned}
& \boldsymbol{e}_{i}^{\prime} \boldsymbol{M} \boldsymbol{e}_{K} \\
& =\boldsymbol{e}_{i}^{\prime} \boldsymbol{M}\left(\mathbf{1}-\sum_{j=1}^{K-1} \boldsymbol{e}_{j}\right)=-\sum_{j=1}^{K-1} \boldsymbol{e}_{i}^{\prime} \boldsymbol{M} \boldsymbol{e}_{j}=-\sum_{j=1}^{K-1} \boldsymbol{e}_{i}^{\prime}\left(\sum_{l=1}^{K-1} \sum_{k=1}^{K-1} \alpha_{k l} \boldsymbol{E}_{k l}\right) \boldsymbol{e}_{j} \\
& =\boldsymbol{e}_{i}^{\prime}\left(\sum_{l=1}^{K-1} \sum_{k=1}^{K-1} \alpha_{k l} \boldsymbol{E}_{k l}\right)\left(\mathbf{1}-\sum_{j=1}^{K-1} \boldsymbol{e}_{j}\right)=\boldsymbol{e}_{i}^{\prime}\left(\sum_{l=1}^{K-1} \sum_{k=1}^{K-1} \alpha_{k l} \boldsymbol{E}_{k l}\right) \boldsymbol{e}_{K}
\end{aligned}
$$

for $1 \leq i \leq K$. 
Proof of Theorem 2.3. Existence of a decomposition of the form (10) follows from the fact that $\boldsymbol{N}-\boldsymbol{N}_{0}$ is a $K \times K$ matrix with every row sum equal to zero, and that the $K(K-1)$-dimensional linear space of all such matrices is spanned by the linearly independent basis matrices $\boldsymbol{H}_{1}, \ldots, \boldsymbol{H}_{K-1}$ and $\boldsymbol{E}_{i j}, i, j=1,2, \ldots, K-1$.

It follows from the general decomposition (10) that

$$
\mathbf{1}^{\prime}\left(\boldsymbol{N}-\boldsymbol{N}_{0}\right) \boldsymbol{e}_{j}= \begin{cases}-K \theta_{j} & \text { for } j=1, \\ K\left(-\theta_{j}+\theta_{j-1}\right) & \text { for } j=2,3, \ldots, K-1, \\ K \theta_{j-1} & \text { for } j=K .\end{cases}
$$

Thus, we have the equations $\theta_{1}=\left(n_{1},-n_{.1}\right) / K$ and

$$
\theta_{j}-\theta_{j-1}=\left(n_{j}-n_{\cdot j}\right) / K \text { for } j=2,3, \ldots, K-1 .
$$

These $K-1$ equations are equivalent to (11), while the remaining equation, $\theta_{K-1}=\left(n_{\cdot K}-n_{K}\right) / K$, is consistent with these, since $\sum_{j=1}^{K} n_{\cdot j}=n=$ $\sum_{j=1}^{K} n_{j}$.

Once the $\theta_{j}$ s are chosen as above, the $\alpha_{i j}$ s are uniquely obtained from Theorem 2.1 by replacing the matrix $\boldsymbol{M}$ with $\boldsymbol{N}-\boldsymbol{N}_{0}-\sum_{j=1}^{K-1} \theta_{j} \boldsymbol{H}_{j}$.

Proof of Theorem 4.1. For a measure $m$ with the stated properties,

$$
\begin{aligned}
m\left(a \boldsymbol{K}_{i j k}\right) & =|a| m\left(\boldsymbol{K}_{i j k}\right) \quad \text { by LH and SYM } \\
& =|a| m\left(\boldsymbol{K}_{11 k}\right) \quad \text { by TI } \\
& =|a|\left[m\left(\boldsymbol{K}_{111}\right)+m\left(\boldsymbol{K}_{121}\right)+\cdots+m\left(\boldsymbol{K}_{1 k 1}\right)\right] \quad \text { by PIN } \\
& =|a| k m\left(\boldsymbol{K}_{111}\right) \quad \text { by TI. }
\end{aligned}
$$

This proves the necessity of the given form. On the other hand, if $m\left(a \boldsymbol{K}_{i j k}\right)=$ $c|a| k$ for some positive constant $c$, then the properties SYM, PIN, TI and LH are satisfied. This completes the proof.

Proof of Theorem 4.2. In view of IIR, we work with $g$. First note that by NOM $g(0,0, \ldots, 0)=0$. Therefore, for a measure satisfying all the stated properties,

$$
\begin{aligned}
g( & m\left(\xi_{111} \boldsymbol{K}_{111}\right), \ldots, m\left(\xi_{11, K-1} \boldsymbol{K}_{11, K-1}\right), \ldots, m\left(\xi_{1, K-1,1} \boldsymbol{K}_{1, K-1,1}\right), \\
& \ldots, m\left(\xi_{K 11} \boldsymbol{K}_{K 11}\right), \ldots, m\left(\xi_{K 1, K-1} \boldsymbol{K}_{K 1, K-1}\right), \ldots, m\left(\xi_{K, K-1,1} \boldsymbol{K}_{K, K-1,1}\right) \\
= & g(0, \ldots, 0)+\alpha \sum_{i=1}^{K} \sum_{j=1}^{K-1} \sum_{k=1}^{K-j} m\left(\xi_{i j k} \boldsymbol{K}_{i j k}\right) \text { for some } \alpha, \text { by repeated use of ERI } \\
\propto & \sum_{i=1}^{K} \sum_{j=1}^{K-1} \sum_{k=1}^{K-j} m\left(\xi_{i j k} \boldsymbol{K}_{i j k}\right) \quad \text { by NOM } \\
\propto & \sum_{i=1}^{K} \sum_{j=1}^{K-1} \sum_{k=1}^{K-j} k\left|\xi_{i j k}\right| \quad \text { by Theorem 4.1. }
\end{aligned}
$$


This proves the necessity part. The fact that a measure proportional to the last expression satisfies all the requisite properties is easy to verify.

Proof of Theorem 4.3. For a typical weighted sum of the form (3),

$$
\begin{aligned}
\boldsymbol{N}-\boldsymbol{N}_{0} & =\sum_{i=1}^{K} \sum_{j=1}^{K-1} \sum_{k=1}^{K-j} \xi_{i j k} \boldsymbol{K}_{i j k}=\sum_{i=1}^{K} \sum_{j=1}^{K-1} \sum_{k=1}^{K-j} \xi_{i j k} \sum_{l=j}^{j+k-1} \boldsymbol{G}_{i l} \\
& =\sum_{i=1}^{K} \sum_{j=1}^{K-1} \sum_{l=j}^{K-1} \sum_{k=l-j+1}^{K-j} \xi_{i j k} \boldsymbol{G}_{i l}=\sum_{i=1}^{K} \sum_{l=1}^{K-1} \sum_{j=1}^{l} \sum_{k=l-j+1}^{K-j} \xi_{i j k} \boldsymbol{G}_{i l} \\
& =\sum_{i=1}^{K} \sum_{l=1}^{K-1} \eta_{i l} \boldsymbol{G}_{i l} .
\end{aligned}
$$

By uniqueness of the representation given in Theorem 2.1, we infer that

$$
\eta_{i l}=\sum_{j=1}^{l} \sum_{k=l-j+1}^{K-j} \xi_{i j k}, \quad i=1, \ldots, K, l=1, \ldots, K-1 .
$$

It follows that

$$
\begin{aligned}
\sum_{i=1}^{K} \sum_{l=1}^{K-1}\left|\eta_{i l}\right| & =\sum_{i=1}^{K} \sum_{l=1}^{K-1}\left|\sum_{j=1}^{l} \sum_{k=l-j+1}^{K-j} \xi_{i j k}\right| \\
& \leq \sum_{i=1}^{K} \sum_{l=1}^{K-1} \sum_{j=1}^{l} \sum_{k=l-j+1}^{K-j}\left|\xi_{i j k}\right|=\sum_{i=1}^{K} \sum_{j=1}^{K-1} \sum_{l=j}^{K-1} \sum_{k=l-j+1}^{K-j}\left|\xi_{i j k}\right| \\
& =\sum_{i=1}^{K} \sum_{j=1}^{K-1} \sum_{k=1}^{K-j} \sum_{l=j}^{k+j-1}\left|\xi_{i j k}\right|=\sum_{i=1}^{K} \sum_{j=1}^{K-1} \sum_{k=1}^{K-j} k\left|\xi_{i j k}\right| .
\end{aligned}
$$

This proves part (a). In view of this result, the definition of MIN and Theorem 4.2, part (b) can be proved by showing the equivalence of the two expressions of $M_{B}$ in (23). This follows from Theorem 4.7, proved below.

Proof of Theorem 4.4. The proof follows along the lines of the proof of Theorem 4.1, with the axiom DR replacing SYM.

Proof of Theorem 4.5. The proof follows along the lines of that of Theorem 4.2, with the reference to Theorem 4.1 replaced by a similar reference to Theorem 4.4 .

Proof of Theorem 4.6. It follows from (52), obtained as part of the proof of Theorem 4.3, that for any representation of $\boldsymbol{N}-\boldsymbol{N}_{0}$ of the form (3),

$$
\sum_{i=1}^{K} \sum_{l=1}^{K-1} \eta_{i l}=\sum_{i=1}^{K} \sum_{l=1}^{K-1} \sum_{j=1}^{l} \sum_{k=l-j+1}^{K-j} \xi_{i j k}=\sum_{i=1}^{K} \sum_{j=1}^{K-1} \sum_{l=j}^{K-1} \sum_{k=l-j+1}^{K-j} \xi_{i j k},
$$


which further simplifies to

$$
\sum_{i=1}^{K} \sum_{l=1}^{K-1} \eta_{i l}=\sum_{i=1}^{K} \sum_{j=1}^{K-1} \sum_{k=1}^{K-j} \sum_{l=j}^{k+j-1} \xi_{i j k}=\sum_{i=1}^{K} \sum_{j=1}^{K-1} \sum_{k=1}^{K-j} k \xi_{i j k} .
$$

This proves part (a). The equivalence of the two expressions of $M_{S}$ in (24) is established in Theorem 4.7, proved below. Chattopadhyay et al. (2019) have already proved that the measure $M_{S}$ lies between -1 and 1 . Hence, the statement of part (b) follows from part (a) and Theorem 4.5.

Proof of Theorem 4.7. Note from Theorem 2.1 that

$$
\begin{aligned}
\eta_{i j} & =\eta_{i, j-1}-\left(n_{i j}-n_{i} . \boldsymbol{e}_{i}^{\prime} \boldsymbol{e}_{j}\right)=-\sum_{l=1}^{j}\left(n_{i j}-n_{i} \cdot \boldsymbol{e}_{i}^{\prime} \boldsymbol{e}_{l}\right) \\
& =\left\{\begin{array}{ll}
-\sum_{l=1}^{j} n_{i j} & \text { if } j<i, \\
-\sum_{l=1}^{j} n_{i j}-n_{i} . & \text { if } j \geq i,
\end{array}= \begin{cases}-\sum_{l=1}^{j} n_{i j} & \text { if } j<i, \\
\sum_{l=j+1}^{K} n_{i j} & \text { if } j \geq i .\end{cases} \right.
\end{aligned}
$$

Therefore, $\eta_{i j}=\left|\eta_{i j}\right|$ for $j \geq i$ and $\eta_{i j}=-\left|\eta_{i j}\right|$ for $j<i$. Further, it follows from (25) that

$$
\begin{aligned}
& n M_{B U}=\sum_{i=2}^{K} \sum_{j=1}^{i-1} n_{i j}(i-j)=\sum_{i=2}^{K} \sum_{j=1}^{i-1} \sum_{l=j}^{i-1} n_{i j} \\
& =\sum_{i=2}^{K} \sum_{l=1}^{i-1} \sum_{j=1}^{l} n_{i j}=-\sum_{i=2}^{K} \sum_{l=1}^{i-1} \eta_{i l}=-\sum_{i=1}^{K} \sum_{\substack{j=1 \\
j<i}}^{K-1} \eta_{i j}=\sum_{i=1}^{K} \sum_{\substack{j=1 \\
j<i}}^{K-1}\left|\eta_{i j}\right| .
\end{aligned}
$$

Likewise, it follows from (26) that

$$
\begin{aligned}
& n M_{B D}=\sum_{i=1}^{K-1} \sum_{j=i+1}^{K} n_{i j}(j-i)=\sum_{i=1}^{K-1} \sum_{j=i+1}^{K} \sum_{l=i}^{j-1} n_{i j} \\
& =\sum_{i=1}^{K-1} \sum_{l=i}^{K-1} \sum_{j=l+1}^{K} n_{i j}=\sum_{i=1}^{K-1} \sum_{l=i}^{K-1} \eta_{i l}=\sum_{i=1}^{K-1} \sum_{\substack{j=1 \\
j \geq i}}^{K-1} \eta_{i j}=\sum_{i=1}^{K-1} \sum_{\substack{j=1 \\
j \geq i}}^{K-1}\left|\eta_{i j}\right| .
\end{aligned}
$$

We also have from (27)

$$
\begin{aligned}
& M_{B}=M_{B U}+M_{B D}=\frac{1}{n} \sum_{i=1}^{K} \sum_{j=1}^{K-1}\left|\eta_{i j}\right| \\
& M_{S}=\frac{1}{K-1}\left(M_{B U}-M_{B D}\right),=-\frac{1}{n(K-1)} \sum_{i=1}^{K} \sum_{j=1}^{K-1} \eta_{i j} .
\end{aligned}
$$

This completes the proof. 
Proof of Theorem 4.8. We can expand the expression (25), by making use of (17), as

$$
M_{B U}=\frac{1}{n} \sum_{i=2}^{K} \sum_{j=1}^{i-1}(i-j) \boldsymbol{e}_{i}^{\prime}\left(\boldsymbol{N}_{0}+\boldsymbol{N}_{1}+\boldsymbol{N}_{2}\right) \boldsymbol{e}_{j} .
$$

It may be verified that

$\frac{1}{n} \sum_{i=1}^{K-1} \sum_{j=i+1}^{K}(j-i) \boldsymbol{e}_{i}^{\prime} \boldsymbol{N}_{0} \boldsymbol{e}_{j}=0, \quad$ and also $\frac{1}{n} \sum_{i=1}^{K-1} \sum_{j=i+1}^{K}(j-i) \boldsymbol{e}_{i}^{\prime} \boldsymbol{E}_{i^{\prime} j^{\prime}} \boldsymbol{e}_{j}=0$

whenever $j^{\prime} \neq i^{\prime}$. Therefore,

$$
\begin{aligned}
M_{B U}= & 0+\frac{1}{n K} \sum_{i=2}^{K} \sum_{j=1}^{i-1}(i-j)\left(n_{\cdot j}-n_{j} .\right) \\
& +\frac{1}{n} \sum_{i=2}^{K} \sum_{j=1}^{i-1}(i-j) \boldsymbol{e}_{i}^{\prime}\left(\sum_{i^{\prime}=1}^{K-1} \alpha_{i^{\prime} i^{\prime}} \boldsymbol{E}_{i^{\prime} i^{\prime}}\right) \boldsymbol{e}_{j} \\
= & \frac{1}{n K} \sum_{j=1}^{K-1} \sum_{i=j+1}^{K}(i-j)\left(n_{\cdot j}-n_{j}\right)+\frac{1}{n} \sum_{i^{\prime}=1}^{K-1} \alpha_{i^{\prime} i^{\prime}}
\end{aligned}
$$

(as non-zero term occurs in second sum only for $i-1=j=i^{\prime}$ )

$$
\begin{aligned}
= & \frac{1}{n K} \sum_{j=1}^{K-1}\left(\frac{K(K+1)}{2}-\frac{j(j+1)}{2}-j(K-j)\right)\left(n_{\cdot j}-n_{j}\right)+\frac{1}{n} \sum_{i=1}^{K-1} \alpha_{i i} \\
= & \frac{1}{2 n} \sum_{j=1}^{K}\left((K-j)^{2}+K-j\right)\left(\theta_{j-1}-\theta_{j}\right)+\frac{1}{n} \sum_{i=1}^{K-1} \alpha_{i i} \quad\left(\text { where } \theta_{0}=0\right) \\
= & \frac{1}{2 n}\left[\sum_{j=2}^{K}\left((K-j)^{2}-(K-j)\right) \theta_{j-1}-\sum_{j=1}^{K-1}\left((K-j)^{2}-(K-j)\right) \theta_{j}\right] \\
& +\frac{1}{n} \sum_{i=1}^{K-1} \alpha_{i i} \\
= & \frac{1}{2 n}\left[\sum_{j=1}^{K-1}\left((K-j-1)^{2}-(K-j-1)\right) \theta_{j}\right. \\
& \left.-\sum_{j=1}^{K-1}\left((K-j)^{2}-(K-j)\right) \theta_{j}\right]+\frac{1}{n} \sum_{i=1}^{K-1} \alpha_{i i} \\
= & -\frac{1}{n} \sum_{j=1}^{K-1}(K-j) \theta_{j}+\frac{1}{n} \sum_{i=1}^{K-1} \alpha_{i i} .
\end{aligned}
$$


As for the measure of downward mobility, (26) can simplified as

$$
\begin{aligned}
& M_{B D}=\frac{1}{n} \sum_{i=1}^{K-1} \sum_{j=i+1}^{K}(j-i) \boldsymbol{e}_{i}^{\prime}\left(\boldsymbol{N}_{0}+\boldsymbol{N}_{1}+\boldsymbol{N}_{2}\right) \boldsymbol{e}_{j} \\
& =0+\frac{1}{n K} \sum_{i=1}^{K-1} \sum_{j=i+1}^{K}(j-i)\left(n_{\cdot j}-n_{j} .\right) \\
& +\frac{1}{n} \sum_{i=1}^{K-1} \sum_{j=i+1}^{K}(j-i) \boldsymbol{e}_{i}^{\prime}\left(\sum_{i^{\prime}=1}^{K-1} \alpha_{i^{\prime} i^{\prime}} \boldsymbol{E}_{i^{\prime} i^{\prime}}\right) \boldsymbol{e}_{j}, \\
& =\frac{1}{n K} \sum_{j=2}^{K} \sum_{i=1}^{j-1}(j-i)\left(n_{\cdot j}-n_{j}\right)+\frac{1}{n} \sum_{i=1}^{K-1} \sum_{j=i+1}^{i+1}(j-i) \alpha_{i i} \\
& =\frac{1}{n K} \sum_{j=1}^{K} \sum_{i=1}^{j}(j-i)\left(n_{\cdot j}-n_{j}\right)+\frac{1}{n} \sum_{i=1}^{K-1} \alpha_{i i} \\
& =\frac{1}{n K} \sum_{j=1}^{K}\left(j^{2}-\frac{j(j+1)}{2}\right)\left(n_{\cdot j}-n_{j}\right)+\frac{1}{n} \sum_{i=1}^{K-1} \alpha_{i i} \\
& =\frac{1}{2 n} \sum_{j=1}^{K}\left(j^{2}-j\right)\left(\theta_{j-1}-\theta_{j}\right)+\frac{1}{n} \sum_{i=1}^{K-1} \alpha_{i i} \quad\left(\text { where } \theta_{0}=0\right) \\
& =\frac{1}{2 n}\left[\sum_{j=1}^{K}\left(j^{2}-j\right) \theta_{j-1}-\sum_{j=1}^{K}\left(j^{2}-j\right) \theta_{j}\right]+\frac{1}{n} \sum_{i=1}^{K-1} \alpha_{i i} \\
& =\frac{1}{2 n}\left[\sum_{j=1}^{K-1}\left((j+1)^{2}-(j+1)\right) \theta_{j}-\sum_{j=1}^{K-1}\left(j^{2}-j\right) \theta_{j}\right]+\frac{1}{n} \sum_{i=1}^{K-1} \alpha_{i i} \\
& =\frac{1}{n} \sum_{j=1}^{K-1} j \theta_{j}+\frac{1}{n} \sum_{i=1}^{K-1} \alpha_{i i} .
\end{aligned}
$$

The expressions for $M_{B}$ and $M_{S}$ are obtained by combining those of $M_{B U}$ and $M_{B D}$.

Proof of Theorem 4.9. Using the properties repeatedly, we have for $k=1, \ldots, K-j, j=1, \ldots, K-1$ and any non-negative $a$,

$$
\begin{aligned}
m\left(a \boldsymbol{L}_{j k}\right) & =a m\left(\boldsymbol{L}_{j k}\right) \quad \text { using LH } \\
& =a m\left(\boldsymbol{L}_{j 1}\right)+a m\left(\boldsymbol{L}_{j+1,1}\right)+\cdots+a m\left(\boldsymbol{L}_{j+k-1,1}\right) \quad \text { using PIN } \\
& =a k m\left(\boldsymbol{L}_{11}\right) \quad \text { using TI } \\
& =-a k\left|m\left(\boldsymbol{L}_{11}\right)\right| \quad \text { using DR. }
\end{aligned}
$$

For negative $a$, one can write $a=-|a|$ and use DR together with the above steps to prove the assertion. The converse is easy to verify. 
Proof of Theorem 4.10. We begin with the necessity of the given form of the specified measure, say $m$. The given conditions imply, by Theorem 4.9, that $m\left(a \boldsymbol{L}_{j k}\right)=-c a k$ for some positive constant $c$. In view of IIR, we can work with $g$ instead of $m$. NOM implies that $g(0,0, \ldots, 0)=0$. By using ERI repeatedly, we have

$$
\begin{aligned}
& g\left(m_{1}, m_{2}, \ldots, m_{K(K-1) / 2}\right) \\
& \quad=g(0,0, \ldots, 0)-c\left(m_{1}+m_{2}+\cdots+m_{K(K-1) / 2}\right) \\
& \quad=-c\left(m_{1}+m_{2}+\cdots+m_{K(K-1) / 2}\right) .
\end{aligned}
$$

Thus, we have from IIR and Theorem 4.9

$$
\begin{aligned}
& m\left(\sum_{j=1}^{K-1} \sum_{k=1}^{K-j} \phi_{j k} \boldsymbol{L}_{j k}\right) \\
&= g\left(m\left(\phi_{11} \boldsymbol{L}_{11}\right), \ldots, m\left(\phi_{1, K-1} \boldsymbol{L}_{1, K-1}\right), \ldots,\right. \\
&\left.m\left(\phi_{K-2,1} \boldsymbol{L}_{K-2,1}\right), m\left(\phi_{K-2,2} \boldsymbol{L}_{K-2,2}\right), m\left(\phi_{K-1,1} \boldsymbol{L}_{K-1,1}\right)\right) \\
&=-c \sum_{j=1}^{K-1} \sum_{k=1}^{K-j} k m\left(\phi_{j k} \boldsymbol{L}_{j k}\right)=-c \sum_{j=1}^{K-1} \sum_{k=1}^{K-j} k \phi_{j k}
\end{aligned}
$$

for some $c>0$. The sufficiency of the given form of $m$ is easy to verify.

Proof of Theorem 4.11. By using the fact $\boldsymbol{L}_{j k}=\sum_{l=j}^{j+k+1} \boldsymbol{H}_{l}$, we have

$$
\begin{aligned}
\boldsymbol{N}_{1} & =\sum_{j=1}^{K-1} \sum_{k=1}^{K-j} \phi_{j k} \boldsymbol{L}_{j k}=\sum_{j=1}^{K-1} \sum_{k=1}^{K-j} \phi_{j k} \sum_{l=j}^{j+k-1} \boldsymbol{H}_{l} \\
& =\sum_{j=1}^{K-1} \sum_{l=j}^{K-1} \sum_{k=l-j+1}^{K-j} \phi_{j k} \boldsymbol{H}_{l}=\sum_{l=1}^{K-1} \sum_{j=1}^{l} \sum_{k=l-j+1}^{K-j} \phi_{j k} \boldsymbol{H}_{l} .
\end{aligned}
$$

By the uniqueness of the representation $\boldsymbol{N}_{1}=\sum_{j=1}^{K-1} \theta_{j} \boldsymbol{H}_{j}$ given in Theorem 2.3, we conclude that $\theta_{l}=\sum_{j=1}^{l} \sum_{k=l-j+1}^{K-j} \phi_{j k}$. Therefore,

$$
\begin{aligned}
\sum_{l=1}^{K-1} \theta_{l} & =\sum_{l=1}^{K-1} \sum_{j=1}^{l} \sum_{k=l-j+1}^{K-j} \phi_{j k}=\sum_{j=1}^{K-1} \sum_{l=j}^{K-1} \sum_{k=l-j+1}^{K-j} \phi_{j k} \\
& =\sum_{j=1}^{K-1} \sum_{k=1}^{K-j} \sum_{l=j}^{k+j-1} \phi_{j k}=\sum_{j=1}^{K-1} \sum_{k=1}^{K-j} k \phi_{j k} .
\end{aligned}
$$

This proves part (a). It also follows from the above steps that

$$
\sum_{j=1}^{K-1}\left|\theta_{j}\right|=\sum_{l=1}^{K-1}\left|\sum_{j=1}^{l} \sum_{k=l-j+1}^{K-j} \phi_{j k}\right| \leq \sum_{l=1}^{K-1} \sum_{j=1}^{l} \sum_{k=l-j+1}^{K-j}\left|\phi_{j k}\right|=\sum_{j=1}^{K-1} \sum_{k=1}^{K-j} k\left|\phi_{j k}\right| .
$$


This proves part (b). In order to prove part (c), we use (11) to obtain

$$
\begin{aligned}
\sum_{j=1}^{K-1} \theta_{j} & =\frac{1}{K} \sum_{j=1}^{K-1} \sum_{i=1}^{j}\left(n_{i \cdot}-n_{\cdot i}\right) \\
& =\frac{1}{K} \sum_{i=1}^{K-1} \sum_{j=i}^{K-1}\left(n_{i \cdot}-n_{\cdot i}\right)=\frac{1}{K} \sum_{i=1}^{K}(K-i)\left(n_{i \cdot}-n_{\cdot i}\right) \\
& =\sum_{i=1}^{K}\left(n_{i \cdot}-n_{\cdot i}\right)+\frac{1}{K} \sum_{i=1}^{K} i\left(n_{\cdot i}-n_{i} .\right)=\frac{1}{K} \sum_{i=1}^{K} i\left(n_{\cdot i}-n_{i \cdot}\right) .
\end{aligned}
$$

This completes the proof.

Proof of Theorem 4.12. Let the properties DR, LH, PIN and TI hold for a measure $m$. We have for $k=1, \ldots, K-i, i=1, \ldots, K-1$, $l=1, \ldots, K-j, j=1, \ldots, K-1$ and any real $a$,

$$
\begin{aligned}
m\left(a \boldsymbol{F}_{i j k l}\right)= & a m\left(\boldsymbol{F}_{i j k l}\right) \quad \text { using DR and LH } \\
= & a m\left(\boldsymbol{F}_{i j 1 l}\right)+a m\left(\boldsymbol{F}_{i+1, j 1 l}\right)+\cdots+a m\left(\boldsymbol{L}_{i+k-1, j 1 l}\right) \text { using PIN } \\
= & {\left[a m\left(\boldsymbol{F}_{i j 11}\right)+a m\left(\boldsymbol{F}_{i, j+1,11}\right)+\cdots+a m\left(\boldsymbol{F}_{i, j+l-1,11}\right)\right] } \\
& +\left[a m\left(\boldsymbol{F}_{i+1, j 11}\right)+a m\left(\boldsymbol{F}_{i+1, j+1,11}\right)+\cdots+a m\left(\boldsymbol{F}_{i+1, j+l-1,11}\right)\right] \\
& +\cdots+\left[a m\left(\boldsymbol{F}_{i+k-1, j 11}\right)+a m\left(\boldsymbol{F}_{i+k-1, j+1,11}\right)\right. \\
& \left.+\cdots+a m\left(\boldsymbol{F}_{i+k-1, j+l-1,11}\right)\right] \quad \text { using PIN } \\
= & a k l m\left(\boldsymbol{F}_{1111}\right) \quad \text { using TI, } \\
= & a k l\left|m\left(\boldsymbol{F}_{1111}\right)\right| \text { using DR, }
\end{aligned}
$$

which proves the assertion. The reverse implication is easy to verify.

Proof of Theorem 4.13. In order to prove the necessity of the given form of the measure $m$, let the conditions of Theorem 4.12 be satisfied (along with IIR, NOM and ERI), so that $m\left(a \boldsymbol{F}_{i j k l}\right)=c a k l$ for some positive constant $c$. In view of IIR, we can work with $g$ instead of $m$. NOM implies that $g(0,0, \ldots, 0)=0$. By using ERI repeatedly, we have

$$
\begin{aligned}
g\left(m_{1}, m_{2}, \ldots, m_{K^{2}(K-1)^{2} / 4}\right) & =g(0,0, \ldots, 0)+c\left(m_{1}+m_{2}+\cdots+m_{K^{2}(K-1)^{2} / 4}\right) \\
& =c\left(m_{1}+m_{2}+\cdots+m_{K^{2}(K-1)^{2} / 4}\right) .
\end{aligned}
$$

Thus, from IIR,

$$
\begin{aligned}
m & \left(\sum_{i=1}^{K-1} \sum_{j=1}^{K-1} \sum_{k=1}^{K-i} \sum_{l=1}^{K-j} \beta_{i j k l} \boldsymbol{F}_{i j k l}\right) \\
= & g\left(m\left(\beta_{1111} \boldsymbol{F}_{1111}\right), \ldots, m\left(\beta_{K-2,121} \boldsymbol{F}_{K-2,121}\right), m\left(\beta_{K-1,111} \boldsymbol{F}_{K-1,111}\right),\right. \\
& \left.\quad \ldots, m\left(\beta_{K-1, K-1,11} \boldsymbol{F}_{K-1, K-1,11}\right)\right) \\
= & c \sum_{i=1}^{K-1} \sum_{j=1}^{K-1} \sum_{k=1}^{K-i} \sum_{l=1}^{K-j} k \beta_{i j k l} .
\end{aligned}
$$


The sufficiency can be checked easily by direct verification of the list of properties.

Proof of Theorem 4.14. By using (8), we obtain

$$
\begin{aligned}
\boldsymbol{N}_{2} & =\sum_{i=1}^{K-1} \sum_{j=1}^{K-1} \sum_{k=1}^{K-i} \sum_{l=1}^{K-j} \beta_{i j k l} \boldsymbol{F}_{i j k l} \\
& =\sum_{i=1}^{K-1} \sum_{k=1}^{K-i} \sum_{j=1}^{K-1} \sum_{l=1}^{K-j} \beta_{i j k l} \sum_{i^{\prime}=i}^{i+k-1} \sum_{j^{\prime}=j}^{j+l-1} \boldsymbol{E}_{i^{\prime} j^{\prime}} \\
& =\sum_{i=1}^{K-1} \sum_{i^{\prime}=i}^{K-1} \sum_{k=i^{\prime}-i+1}^{K-i} \sum_{j=1}^{K-1} \sum_{j^{\prime}=j}^{K-1} \sum_{l=j^{\prime}-j+1}^{K-j} \beta_{i j k l} \boldsymbol{E}_{i^{\prime} j^{\prime}} \\
& =\sum_{i^{\prime}=1}^{K-1} \sum_{i=1}^{i^{\prime}} \sum_{k=i^{\prime}-i+1}^{K-i} \sum_{j^{\prime}=1}^{K-1} \sum_{j=1}^{j^{\prime}} \sum_{l=j^{\prime}-j+1}^{K-j} \beta_{i j k l} \boldsymbol{E}_{i^{\prime} j^{\prime}} \\
& =\sum_{i^{\prime}=1}^{K-1} \sum_{j^{\prime}=1}^{K-1} \sum_{i=1}^{i^{\prime}} \sum_{k=i^{\prime}-i+1}^{K-i} \sum_{j=1}^{j^{\prime}} \sum_{l=j^{\prime}-j+1}^{K-j} \beta_{i j k l} \boldsymbol{E}_{i^{\prime} j^{\prime}} .
\end{aligned}
$$

However, $\boldsymbol{N}_{2}$ is uniquely decomposed as $\sum_{i^{\prime}=1}^{K-1} \sum_{j^{\prime}=1}^{K-1} \alpha_{i^{\prime} j^{\prime}} \boldsymbol{E}_{i^{\prime} j^{\prime}}$ according to Theorem 2.2, i.e., $\alpha_{i^{\prime} j^{\prime}}=\sum_{i=1}^{i^{\prime}} \sum_{k=i^{\prime}-i+1}^{K-i} \sum_{j=1}^{j^{\prime}} \sum_{l=j^{\prime}-j+1}^{K-j} \beta_{i j k l}$. Hence, by retracing the above sequence of interchange of summations, we have

$$
\begin{aligned}
\sum_{i^{\prime}=1}^{K-1} \sum_{j^{\prime}=1}^{K-1} \alpha_{i^{\prime} j^{\prime}} & =\sum_{i^{\prime}=1}^{K-1} \sum_{j^{\prime}=1}^{K-1} \sum_{i=1}^{i^{\prime}} \sum_{k=i^{\prime}-i+1}^{K-i} \sum_{j=1}^{j^{\prime}} \sum_{l=j^{\prime}-j+1}^{K-j} \beta_{i j k l} \\
& =\sum_{i=1}^{K-1} \sum_{k=1}^{K-i} \sum_{j=1}^{K-1} \sum_{l=1}^{K-j} \sum_{i^{\prime}=i}^{i+k-1} \sum_{j^{\prime}=j}^{j+l-1} \beta_{i j k l} \\
& =\sum_{i=1}^{K-1} \sum_{k=1}^{K-i} \sum_{j=1}^{K-1} \sum_{l=1}^{K-j} k l \beta_{i j k l} .
\end{aligned}
$$

In order to prove part (b), we proceed similarly to obtain

$$
\begin{aligned}
\sum_{i^{\prime}=1}^{K-1} \sum_{j^{\prime}=1}^{K-1}\left|\alpha_{i^{\prime} j^{\prime}}\right| & =\sum_{i^{\prime}=1}^{K-1} \sum_{j^{\prime}=1}^{K-1}\left|\sum_{i=1}^{i^{\prime}} \sum_{k=i^{\prime}-i+1}^{K-i} \sum_{j=1}^{j^{\prime}} \sum_{l=j^{\prime}-j+1}^{K-j} \beta_{i j k l}\right| \\
& \leq \sum_{i^{\prime}=1}^{K-1} \sum_{j^{\prime}=1}^{K-1} \sum_{i=1}^{i^{\prime}} \sum_{k=i^{\prime}-i+1}^{K-i} \sum_{j=1}^{j^{\prime}} \sum_{l=j^{\prime}-j+1}^{K-j}\left|\beta_{i j k l}\right| \\
& =\sum_{i=1}^{K-1} \sum_{k=1}^{K-i} \sum_{j=1}^{K-1} \sum_{l=1}^{K-j} k l\left|\beta_{i j k l}\right| .
\end{aligned}
$$


For proving part (c), observe

$$
\begin{aligned}
& \sum_{i=1}^{K-1} \sum_{j=1}^{K-1} \alpha_{i j} \\
= & \sum_{i=1}^{K-1} \sum_{j=1}^{K-1} i j \alpha_{i j}-\sum_{i=1}^{K-1} \sum_{j=1}^{K-1} i(j+1) \alpha_{i j} \\
& \quad-\sum_{i=1}^{K-1} \sum_{j=1}^{K-1}(i+1) j \alpha_{i j}+\sum_{i=1}^{K-1} \sum_{j=1}^{K-1}(i+1)(j+1) \alpha_{i j} \\
= & \sum_{i=1}^{K-1} \sum_{j=1}^{K-1} i j \alpha_{i j}-\sum_{i=1}^{K-1} \sum_{j=2}^{K} i j \alpha_{i, j-1}-\sum_{i=2}^{K} \sum_{j=1}^{K-1} i j \alpha_{i-1, j}+\sum_{i=2}^{K} \sum_{j=2}^{K} i j \alpha_{i-1, j-1} \\
= & \sum_{i=1}^{K} \sum_{j=1}^{K} i j\left(\alpha_{i j}-\alpha_{i, j-1}-\alpha_{i-1, j}+\alpha_{i-1, j-1}\right)=-\sum_{i=1}^{K} \sum_{j=1}^{K} i j \boldsymbol{e}_{i}^{\prime} \boldsymbol{N}_{2} \boldsymbol{e}_{j} .
\end{aligned}
$$

This completes the proof.

Proof of Theorem 4.15. It follows from (34) that

$$
\begin{aligned}
M_{E}= & \left(\frac{4}{3(K-1)}\right)^{2}\left[\frac{K+1}{2}(E(J)-E(I))+E\left(I^{2}\right)-E(I J)\right] \\
= & \left(\frac{4}{3(K-1)}\right)^{2} E\left[\left(I-\frac{K+1}{2}\right)(I-J)\right] \\
= & \left(\frac{4}{3(K-1)}\right)^{2} \sum_{i=1}^{K} E\left[\left(I-\frac{K+1}{2}\right)(I-J) \mid I=i\right] P(I=i) \\
= & \left(\frac{4}{3(K-1)}\right)^{2} \sum_{i=1}^{K}\left(i-\frac{K+1}{2}\right) E(i-J \mid I=i) P(I=i) \\
& +\left(\frac{4}{3(K-1)}\right)^{2} \sum_{i=1}^{K}\left(i-\frac{K+1}{2}\right) E(i-J \mid I=i) P(I=i) \\
\leq & \left(\frac{4}{3(K-1)}\right)^{2} \sum_{i=1}^{K}\left(\frac{K+1}{2}-i\right)(K-i) P(I=i) \\
& +\left(\frac{4}{3(K-1)}\right)^{2} \sum_{i=1}^{K}\left(i-\frac{K+1}{2}\right)(i-1) P(I=i)
\end{aligned}
$$




$$
\begin{aligned}
\leq & \left(\frac{4}{3(K-1)}\right)^{2} \sum_{\substack{i=1 \\
i<(K+1) / 2}}^{K}\left(\frac{K+1}{2}-1\right)(K-1) P(I=i) \\
& \left(\frac{4}{3(K-1)}\right)^{2} \sum_{\substack{i=1 \\
i>(K+1) / 2}}^{K}\left(K-\frac{K+1}{2}\right)(K-1) P(I=i) \\
= & \left(\frac{4}{3(K-1)}\right)^{2} \frac{(K-1)^{2}}{2}\left[\sum_{\substack{i=1 \\
i<(K+1) / 2}}^{K} P(I=i)+\sum_{\substack{i=1 \\
i>(K+1) / 2}}^{K} P(I=i)\right] \leq \frac{8}{9} .
\end{aligned}
$$

Further,

$$
\begin{aligned}
& M_{E}=\left(\frac{4}{3(K-1)}\right)^{2} \sum_{\substack{i=1 \\
i<(K+1) / 2}}^{K}\left(i-\frac{K+1}{2}\right) E(i-J \mid I=i) P(I=i) \\
& +\left(\frac{4}{3(K-1)}\right)^{2} \sum_{\substack{i=1 \\
i>(K+1) / 2}}^{K}\left(i-\frac{K+1}{2}\right) E(i-J \mid I=i) P(I=i) \\
& \geq-\left(\frac{4}{3(K-1)}\right)^{2} \sum_{\substack{i=1 \\
i<(K+1) / 2}}^{K}\left(\frac{K+1}{2}-i\right)(i-1) P(I=i) \\
& -\left(\frac{4}{3(K-1)}\right)^{2} \sum_{\substack{i=1 \\
i>(K+1) / 2}}^{K}\left(i-\frac{K+1}{2}\right)(K-i) P(I=i) \\
& \geq-\left(\frac{4}{3(K-1)}\right)^{2} \sum_{\substack{i=1 \\
i<(K+1) / 2}}^{K}\left(\frac{\frac{K+1}{2}-i+(i-1)}{2}\right)^{2} P(I=i) \\
& -\left(\frac{4}{3(K-1)}\right)^{2} \sum_{\substack{i=1 \\
i>(K+1) / 2}}^{K}\left(\frac{i-\frac{K+1}{2}+(K-i)}{2}\right)^{2} P(I=i) \\
& =-\left(\frac{4}{3(K-1)}\right)^{2}\left(\frac{K-1}{4}\right)^{2}\left[\sum_{\substack{i=1 \\
i<(K+1) / 2}}^{K} P(I=i)-\sum_{\substack{i=1 \\
i>(K+1) / 2}}^{K} P(I=i)\right] \\
& \geq-\left(\frac{4}{3(K-1)}\right)^{2}\left(\frac{K-1}{4}\right)^{2}=-\frac{1}{9} \text {. }
\end{aligned}
$$


This completes the proof of part (a).

The first inequality used in the proof of the upper bound holds with equality if and only if

$$
J= \begin{cases}K & \text { if } I<\frac{K+1}{2} \\ 1 & \text { if } I>\frac{K+1}{2} .\end{cases}
$$

The other two inequalities hold if and only if $P(I=1)+P(I=K)=1$. These conditions hold simultaneously if and only if the transition matrix is of the type mentioned in part (b).

The first inequality used in the proof of the lower bound holds with equality if and only if

$$
J= \begin{cases}1 & \text { if } I<\frac{K+1}{2}, \\ K & \text { if } I>\frac{K+1}{2} .\end{cases}
$$

The other two inequalities hold if and only if $P(I=(K+3) / 4)+P(I=$ $(3 K+1) / 4)=1$. These conditions hold simultaneously if and only if the transition matrix is of the type mentioned in part (c).

Proof of Theorem 4.16. Characterization of the requisite measure as a multiple of $\sum_{i=1}^{K} \sum_{j=1}^{K-1}\left|\eta_{i j}\right|$ has already been established in Theorem 4.3. Equivalence of the two expressions of $M_{I}$ is shown as follows.

$$
\begin{aligned}
\sum_{i=1}^{K} \sum_{j=1}^{K-1}\left|\eta_{i j}\right| & =\sum_{i=1}^{K} \sum_{j=1}^{K-1}\left|\sum_{k=1}^{j} \boldsymbol{e}_{i}^{\prime}\left(\boldsymbol{N}-\frac{1}{K} \boldsymbol{N} \mathbf{1 1 ^ { \prime }}\right) \boldsymbol{e}_{k}\right| \\
& =\sum_{i=1}^{K} \sum_{j=1}^{K-1}\left|\sum_{k=1}^{j}\left(n_{i k}-\frac{n_{i \cdot}}{K}\right)\right|=\sum_{i=1}^{K} \sum_{j=1}^{K-1}\left|\sum_{k=1}^{j} n_{i k}-\frac{j}{K} n_{i \cdot}\right| .
\end{aligned}
$$

The last expression can also be written as $\sum_{i=1}^{K} n_{i} \cdot \sum_{j=1}^{K}\left|\sum_{k=1}^{j} \frac{n_{i k}}{n_{i}}-\frac{j}{K}\right|$. For any fixed $i$, the summand for various values of $j$ may be recognized as the absolute difference between two discrete cumulative distribution functions (CDFs), the second one being the discrete uniform distribution. The sum over $j$ is the absolute area of the gap between the CDFs. This area is minimized when the two distributions coincide (i.e., $n_{i k} / n_{i}$. $=1 / K$ for all $k$ ) and maximized when the non-uniform distribution has all the mass at 1 or $K$ (i.e., either $n_{i 1}$ or $n_{i K}$ is equal to $n_{i}$. and all other $n_{i k} \mathrm{~s}$ are 0 ). The maximized quantity is

$$
\sum_{i=1}^{K} \sum_{j=1}^{K-1} \frac{j}{K} n_{i} .=\frac{K-1}{2} \sum_{i=1}^{K} n_{i}=\frac{n(K-1)}{2} .
$$

This fact justifies the scaling, while the preceding discussion justifies the description of the extreme cases given in the theorem. 
Proof of Theorem 4.17. Since the requisite measure has already been characterized in Theorem 4.5 as a multiple of $\sum_{i=1}^{K} \sum_{j=1}^{K-1} \eta_{i j}$, we only have to show the equivalence of the two expressions of $M_{I S}$ and justify the scaling. Observe that

$$
\begin{aligned}
& -\sum_{i=1}^{K} \sum_{j=1}^{K-1} \eta_{i j} \\
= & \sum_{i=1}^{K} \sum_{j=1}^{K-1} \sum_{k=1}^{j} \boldsymbol{e}_{i}^{\prime}\left(\boldsymbol{N}-\frac{1}{K} \boldsymbol{N} \mathbf{1} \mathbf{1}^{\prime}\right) \boldsymbol{e}_{k}=\sum_{i=1}^{K} \sum_{k=1}^{K-1} \sum_{j=k}^{K-1} \boldsymbol{e}_{i}^{\prime}\left(\boldsymbol{N}-\frac{1}{K} \boldsymbol{N} \mathbf{1} \mathbf{1}^{\prime}\right) \boldsymbol{e}_{k} \\
= & \sum_{k=1}^{K}(K-k) \sum_{i=1}^{K} \boldsymbol{e}_{i}^{\prime}\left(\boldsymbol{N}-\frac{1}{K} \boldsymbol{N 1 1}^{\prime}\right) \boldsymbol{e}_{k} \\
= & \sum_{j=1}^{K}(K-j) \mathbf{1}^{\prime}\left(\boldsymbol{N}-\frac{1}{K} \mathbf{N 1 1}^{\prime}\right) \boldsymbol{e}_{j}=\sum_{j=1}^{K}\left(K n \cdot j-n-j n \cdot j+\frac{j n}{K}\right) \\
= & \sum_{j=1}^{K}\left(\frac{j n}{K}-j n \cdot j\right)=\frac{n(K+1)}{2}-\sum_{j=1}^{K} j n_{\cdot j}=\sum_{j=1}^{K}\left(\frac{K+1}{2}-j\right) n_{\cdot j \cdot} .
\end{aligned}
$$

This proves the equivalence. We only need to check the scaling now. The largest negative value of the last expression occurs when $n_{\cdot K}=n$, and the largest positive value occurs when $n_{\cdot 1}=1$. In each case, the absolute value of the last expression is $n(K-1) / 2$. This justifies the scaling factor.

Proof of Theorem 4.18. In view of the discussion preceding the theorem, we only have to show that the scaling is appropriate and that the two given expressions of $M_{I E}$ coincide. The latter fact is proved from (41) as

$$
\begin{aligned}
-\sum_{k=1}^{K-1} \sum_{l=1}^{K-1} \alpha_{k l} & =\sum_{k=1}^{K-1} \sum_{l=1}^{K-1} \sum_{i=1}^{k} \sum_{j=1}^{l} \boldsymbol{e}_{i}^{\prime} \boldsymbol{N}_{E} \boldsymbol{e}_{j} \\
& =\sum_{k=1}^{K-1} \sum_{l=1}^{K-1} \sum_{i=1}^{k} \sum_{j=1}^{l} \boldsymbol{e}_{i}^{\prime}\left(\boldsymbol{N}-\frac{1}{K} \mathbf{1 1}^{\prime} \boldsymbol{N}-\frac{1}{K} \mathbf{N 1 1}^{\prime}+\frac{n}{K^{2}} \mathbf{1 1}^{\prime}\right) \boldsymbol{e}_{j} \\
& =\sum_{i=1}^{K-1} \sum_{j=1}^{K-1} \sum_{k=i}^{K-1} \sum_{l=j}^{K-1}\left(n_{i j}-\frac{n_{\cdot j}}{K}-\frac{n_{i}}{K}+\frac{n}{K^{2}}\right) \\
& =\sum_{i=1}^{K} \sum_{j=1}^{K}(K-i)(K-j)\left(n_{i j}-\frac{n_{\cdot j}}{K}-\frac{n_{i}}{K}+\frac{n}{K^{2}}\right) \\
& =\sum_{i=1}^{K} \sum_{j=1}^{K} i j\left(n_{i j}-\frac{n_{\cdot j}}{K}-\frac{n_{i \cdot}}{K}+\frac{n}{K^{2}}\right)
\end{aligned}
$$




$$
\begin{aligned}
& =\sum_{i=1}^{K} \sum_{j=1}^{K} i j n_{i j}+\frac{K+1}{2} \sum_{j=1}^{K} j n_{\cdot j}+\frac{K+1}{2} \sum_{i=1}^{K} i n_{i} \cdot-\frac{n(K+1)^{2}}{4} \\
& =\sum_{i=1}^{K} \sum_{j=1}^{K}\left(i-\frac{K+1}{2}\right)\left(j-\frac{K+1}{2}\right) n_{i j} .
\end{aligned}
$$

The values of the factors $\left(i-\frac{K+1}{2}\right)$ and $\left(j-\frac{K+1}{2}\right)$ range from $-\frac{K-1}{2}$ to $\frac{K-1}{2}$. Therefore, the largest negative value of the last expression occurs when all the mass are concentrated on the opposite extreme values of $i$ and $j$, namely $(i, j)=(1, K)$ and $(i, j)=(K, 1)$. Likewise, the largest positive value occurs when all the mass are concentrated on the identical extreme values of $i$ and $j$, namely $(i, j)=(1,1)$ and $(i, j)=(K, K)$. This completes the proof.

Proof of Theorem 5.1. Given IIR, NOM means $g(0, \ldots, 0)=0$. Therefore, IIR and ERI together imply that for some constant $c$,

$$
\begin{aligned}
g\left(m\left(\tau_{111} \boldsymbol{K}_{110}\right), m\left(\tau_{112} \boldsymbol{K}_{111}\right), \ldots, m\left(\tau_{K K K} \boldsymbol{K}_{K K 0}\right)\right) \\
=g(0, \ldots, 0)+c \sum_{i=1}^{K} \sum_{j=1}^{K} \sum_{l=1}^{K} m\left(\tau_{i j l} \boldsymbol{K}_{i j, l-j}\right) \\
\propto \sum_{i=1}^{K} \sum_{j=1}^{K} \sum_{l=1}^{K} m\left(\tau_{i j l} \boldsymbol{K}_{i j, l-j}\right) \quad \text { by NOM } \\
=\sum_{i=1}^{K} \sum_{j=1}^{K} \sum_{l=1}^{K} \tau_{i j l} m\left(\boldsymbol{K}_{i j, l-j}\right) \quad \text { by LH } \\
\propto \sum_{i=1}^{K} \sum_{j=1}^{K} \sum_{l=1}^{K} \tau_{i j l} \quad \text { by UM. }
\end{aligned}
$$

It can be easily verified that, as a measure of $\sum_{i=1}^{K} \sum_{j=1}^{K} \sum_{l=1}^{K} \tau_{i j l} \boldsymbol{K}_{i j, l-j}$, the last expression satisfies all the stated properties.

Proof of Theorem 5.2. In view of Theorem 5.1, we only have to identify the minimum value of the measure given there, and scale it appropriately. Observe that

$$
\begin{aligned}
\sum_{i=1}^{K} \sum_{j=1}^{K} \boldsymbol{e}_{i}^{\prime}\left(\boldsymbol{N}-\boldsymbol{N}_{0}\right)\left(\boldsymbol{e}_{j}-\boldsymbol{e}_{i}\right) & =\sum_{i=1}^{K} \sum_{j=1}^{K}\left(n_{i j}-n_{i} . \boldsymbol{e}_{i}^{\prime} \boldsymbol{e}_{j}-n_{i i}+n_{i} .\right) \\
& =n-n-K \sum_{i=1}^{K} n_{i i}+K n=K\left(n-\sum_{i=1}^{K} n_{i i}\right) .
\end{aligned}
$$

On the other hand, for any expansion of $\boldsymbol{N}-\boldsymbol{N}_{0}$ of the form (43), we can 
write

$$
\begin{aligned}
& \sum_{i=1}^{K} \sum_{j=1}^{K} \boldsymbol{e}_{i}^{\prime}\left(\boldsymbol{N}-\boldsymbol{N}_{0}\right)\left(\boldsymbol{e}_{j}-\boldsymbol{e}_{i}\right) \\
& =\sum_{i=1}^{K} \sum_{j=1}^{K} \boldsymbol{e}_{i}^{\prime}\left(\sum_{i^{\prime}=1}^{K} \sum_{j^{\prime}=1}^{K} \sum_{l=1}^{K} \tau_{i^{\prime} j^{\prime} l} \boldsymbol{K}_{i^{\prime} j^{\prime}, l-j^{\prime}}\right)\left(\boldsymbol{e}_{j}-\boldsymbol{e}_{i}\right) \\
& =\sum_{i=1}^{K} \sum_{j=1}^{K} \boldsymbol{e}_{i}^{\prime}\left(\sum_{i^{\prime}=1}^{K} \sum_{j^{\prime}=1}^{K} \sum_{l=1}^{K} \tau_{i^{\prime} j^{\prime} l} \boldsymbol{e}_{i^{\prime}}\left(\boldsymbol{e}_{l}-\boldsymbol{e}_{j^{\prime}}\right)^{\prime}\right)\left(\boldsymbol{e}_{j}-\boldsymbol{e}_{i}\right) \\
& =\sum_{i=1}^{K} \sum_{j=1}^{K}\left(\sum_{j^{\prime}=1}^{K} \sum_{l=1}^{K} \tau_{i j^{\prime} l}\left(\boldsymbol{e}_{l}-\boldsymbol{e}_{j^{\prime}}\right)^{\prime}\right)\left(\boldsymbol{e}_{j}-\boldsymbol{e}_{i}\right) \\
& =\sum_{i=1}^{K} \sum_{j=1}^{K} \sum_{j^{\prime}=1}^{K} \sum_{l=1}^{K} \tau_{i j^{\prime} l}\left(\boldsymbol{e}_{l}^{\prime} \boldsymbol{e}_{j}-\boldsymbol{e}_{j^{\prime}}^{\prime} \boldsymbol{e}_{j}-\boldsymbol{e}_{l}^{\prime} \boldsymbol{e}_{i}+\boldsymbol{e}_{j^{\prime}}^{\prime} \boldsymbol{e}_{i}\right) \\
& =\sum_{i=1}^{K} \sum_{j=1}^{K} \sum_{j^{\prime}=1}^{K} \tau_{i j^{\prime} j}-\sum_{i=1}^{K} \sum_{j=1}^{K} \sum_{l=1}^{K} \tau_{i j l}-\sum_{i=1}^{K} \sum_{j=1}^{K} \sum_{j^{\prime}=1}^{K} \tau_{i j^{\prime} i}+\sum_{i=1}^{K} \sum_{j=1}^{K} \sum_{l=1}^{K} \tau_{i i l} \\
& =\sum_{i=1}^{K} \sum_{j=1}^{K} \sum_{\substack{l=1 \\
l \neq i}}^{K} \tau_{i i l}-\sum_{i=1}^{K} \sum_{j=1}^{K} \sum_{\substack{j^{\prime}=1 \\
j^{\prime} \neq i}}^{K} \tau_{i j^{\prime} i}=K \sum_{i=1}^{K} \sum_{\substack{l=1 \\
l \neq i}}^{K} \tau_{i i l}-K \sum_{i=1}^{K} \sum_{\substack{j^{\prime}=1 \\
j^{\prime} \neq i}}^{K} \tau_{i j^{\prime} i} \\
& \leq K \sum_{i=1}^{K} \sum_{\substack{l=1 \\
l \neq i}}^{K} \tau_{i i l}+K \sum_{i=1}^{K} \sum_{\substack{j^{\prime}=1 \\
j^{\prime} \neq i}}^{K} \tau_{i j^{\prime} i} \leq K \sum_{i=1}^{K} \sum_{j=1}^{K} \sum_{l=1}^{K} \tau_{i j l}
\end{aligned}
$$

since all the $\tau_{i j l} \mathrm{~s}$ are non-negative. It follows that

$$
\sum_{i=1}^{K} \sum_{j=1}^{K} \sum_{l=1}^{K} \tau_{i j l} \geq\left(n-\sum_{i=1}^{K} n_{i i}\right) .
$$

In order to establish that the right hand side is indeed the minimum value of the left hand side, we have to show that the above holds with equality for some expansion of the form (43). For this purpose we can write

$$
\begin{aligned}
\boldsymbol{N}-\boldsymbol{N}_{0} & =\sum_{i=1}^{K} \sum_{l=1}^{K} n_{i l} \boldsymbol{e}_{i} \boldsymbol{e}_{l}^{\prime}-\sum_{i=1}^{K} n_{i} \cdot \boldsymbol{e}_{i} \boldsymbol{e}_{i}^{\prime}=\sum_{i=1}^{K} \sum_{\substack{l=1 \\
l \neq i}}^{K} n_{i l} \boldsymbol{e}_{i}\left(\boldsymbol{e}_{l}-\boldsymbol{e}_{i}\right)^{\prime} \\
& =\sum_{i=1}^{K} \sum_{\substack{l=1 \\
l \neq i}}^{K} n_{i l} \boldsymbol{K}_{i i, l-i}=\sum_{i=1}^{K} \sum_{j=1}^{K} \sum_{l=1}^{K} \tau_{i j l} \boldsymbol{K}_{i j, l-j},
\end{aligned}
$$


where

$$
\tau_{i j l}= \begin{cases}n_{i l} & \text { if } j=i, l \neq i \\ 0 & \text { otherwise }\end{cases}
$$

The above inequality holds with equality for this special choice of the $\tau_{i j l} \mathrm{~s}$. Therefore, a measure satisfying the requisite properties has to be proportional to $n-\sum_{i=1}^{K} n_{i i}$, which $M_{N}$ is. Appropriateness of the scaling and the conditions for the measure to be equal to be 0 or 1 are easy to verify.

Proof of Theorem 5.3. Instead of $\sum_{j=1}^{K-1} \sum_{k=1}^{K-j} \phi_{j k} \boldsymbol{L}_{j k}$, we work with the equivalent representation $\sum_{j=1}^{K} \sum_{i=1}^{K} \pi_{j i} \boldsymbol{L}_{j, i-j}$. Proceeding as in the proof of Theorem 4.10, we have by virtue of IIR, NOM and ERI

$$
g\left(m_{1}, m_{2}, \ldots, m_{K^{2}}\right)=c_{1}\left(m_{1}+m_{2}+\cdots+m_{K^{2}}\right)
$$

for some constant $c_{1}$. Therefore, from IIR property of the measure $m$

$$
\begin{aligned}
& m\left(\pi_{11} \boldsymbol{L}_{10}, \ldots, \pi_{1 K} \boldsymbol{L}_{1, K-1}, \ldots, \pi_{K 1} \boldsymbol{L}_{K,-K+1}, \ldots, \pi_{K K} \boldsymbol{L}_{K 0}\right) \\
& =c_{1} \sum_{j=1}^{K} \sum_{i=1}^{K} m\left(\pi_{j i} \boldsymbol{L}_{j, i-j}\right) \quad \text { by IIR } \\
& =c_{1} \sum_{j=1}^{K} \sum_{i=1}^{K} \pi_{j i} m\left(\boldsymbol{L}_{j, i-j}\right) \quad \text { by LH } \\
& =c_{1} c_{2} \sum_{j=1}^{K} \sum_{i=1}^{K} \pi_{j i} \quad \text { by UM } \\
& =c_{1} c_{2} \sum_{j=1}^{K-1} \sum_{k=1}^{K-j}\left|\phi_{j k}\right| .
\end{aligned}
$$

for some constant $c_{2}$. This proves the necessity of the stated form of $m$. The sufficiency is easy to verify.

Proof of Theorem 5.4. In view of Theorem 5.3, we only have to identify the minimum value of the measure given there over all possible representations of $\boldsymbol{N}_{1}=\frac{1}{K} \mathbf{1 1}^{\prime}\left(\boldsymbol{N}-\boldsymbol{N}_{0}\right)$ of the form (44), and scale it appropriately.

Let $A$ be the subset of indices $i=1, \ldots, K$ such that $n_{\cdot i}>n_{i}$, and $A^{c}$ be the complementary subset of those indices. Observe that

$$
\begin{aligned}
\mathbf{1}^{\prime} \boldsymbol{N}_{1}\left(\sum_{i \in A} \boldsymbol{e}_{i}\right) & =\frac{1}{K} \mathbf{1}^{\prime} \sum_{j=1}^{N} \mathbf{1}\left(n_{\cdot j}-n_{j}\right) \boldsymbol{e}_{j}^{\prime}\left(\sum_{i \in A} \boldsymbol{e}_{i}\right)=\sum_{i \in A}\left(n_{\cdot i}-n_{i}\right) \\
& =\frac{1}{2} \sum_{i \in A}\left(n_{\cdot i}-n_{i \cdot}\right)+\frac{1}{2} \sum_{i \in A^{c}}\left(n_{i \cdot}-n_{\cdot i}\right)=\frac{1}{2} \sum_{i=1}^{K} \mid n_{\cdot i}-n_{i \cdot} .
\end{aligned}
$$


On the other hand, for any expansion of $\boldsymbol{N}_{1}$ of the form (44), we have

$$
\begin{aligned}
& \mathbf{1}^{\prime} \boldsymbol{N}_{1}\left(\sum_{i \in A} \boldsymbol{e}_{i}\right) \\
& =\mathbf{1}^{\prime} \sum_{j=1}^{K} \sum_{l=1}^{K} \pi_{j l} \mathbf{1}\left(\boldsymbol{e}_{l}-\boldsymbol{e}_{j}\right)^{\prime}\left(\sum_{i \in A} \boldsymbol{e}_{i}\right)=K \sum_{j=1}^{K} \sum_{l=1}^{K} \pi_{j l}\left(\boldsymbol{e}_{l}-\boldsymbol{e}_{j}\right)^{\prime}\left(\sum_{i \in A} \boldsymbol{e}_{i}\right) \\
& =K \sum_{j=1}^{K} \sum_{i \in A}^{K} \pi_{j i}-K \sum_{i \in A}^{K} \sum_{l=1}^{K} \pi_{i l}=K \sum_{j \in A^{c}} \sum_{i \in A}^{K} \pi_{j i}-K \sum_{i \in A}^{K} \sum_{l \in A^{c}} \pi_{i l} \\
& \leq K \sum_{j \in A^{c}} \sum_{i \in A}^{K} \pi_{j i}+K \sum_{i \in A}^{K} \sum_{l \in A^{c}} \pi_{i l} \leq K \sum_{j=1}^{K} \sum_{l=1}^{K} \pi_{j l} .
\end{aligned}
$$

since all the $\pi_{j i}$ s are non-negative. It follows that

$$
\sum_{j=1}^{K} \sum_{l=1}^{K} \pi_{j l} \geq \frac{1}{2 K} \sum_{i=1}^{K}\left|n_{\cdot i}-n_{i \cdot}\right| .
$$

In order to establish that the right hand side is indeed the minimum value of the left hand side, we have to show that the above holds with equality for some expansion of the form (44). For this purpose observe that

$$
\sum_{i \in A}\left(n_{\cdot i}-n_{i}\right)=\sum_{j \in A^{c}}\left(n_{j \cdot}-n_{\cdot j}\right) .
$$

We can arrange the sets of indices in $A$ and $A^{c}$ in the decreasing order of values of $\left(n_{. i}-n_{i}\right.$. ) for $i \in A$ and $\left(n_{j}-n_{. j}\right)$ for $j \in A^{c}$, respectively. If $i_{1}$ and $j_{1}$ happen to be the first indices in the two sets, then we can set aside

$$
\frac{1}{K} \min \left\{n_{\cdot i_{1}}-n_{i_{1}}, n_{j_{1} \cdot}-n_{\cdot j_{1}}\right\} \mathbf{1}\left(\boldsymbol{e}_{i_{1}}-\boldsymbol{e}_{j_{1}}\right)^{\prime}
$$

as the first term of the type $\pi_{j i} \boldsymbol{L}_{j, i-j}$. This depletes both the sets $A$ and $A^{c}$, whose indices can be rearranged again and the process can be repeated for a finite number of times (say, $J$ times) until both sets get exhausted simultaneously. If the $\left(i_{k}, j_{k}\right), k=1, \ldots, J$ are the pairs of first indices obtained in successive steps, it is easy to see that

$$
\boldsymbol{N}_{1}=\sum_{k=1}^{J} \frac{1}{K} \min \left\{n_{\cdot i_{k}}-n_{i_{k}}, n_{j_{k} \cdot}-n_{\cdot j_{k}}\right\} \mathbf{1}\left(\boldsymbol{e}_{i_{k}}-\boldsymbol{e}_{j_{k}}\right)^{\prime}=\sum_{k=1}^{J} \pi_{j_{k} i_{k}} \boldsymbol{L}_{j_{k}, i_{k}-j_{k}}
$$

and

$$
\begin{aligned}
\sum_{k=1}^{J} \pi_{j_{k} i_{k}} & =\sum_{k=1}^{J} \frac{1}{K} \min \left\{n_{\cdot i_{k}}-n_{i_{k} \cdot}, n_{j_{k} \cdot}-n_{\cdot j_{k}}\right\}=\frac{1}{K} \sum_{i \in A}\left(n_{\cdot i}-n_{i \cdot}\right) \\
& =\frac{1}{2 K}\left[\sum_{i \in A}\left(n_{\cdot i}-n_{i \cdot}\right)+\sum_{j \in A^{c}}\left(n_{j \cdot}-n_{\cdot j}\right)\right]=\frac{1}{2 K} \sum_{i \in A}\left|n_{\cdot i}-n_{i \cdot}\right| .
\end{aligned}
$$


Thus, the lower bound is attained.

It is clear that the measure is nonnegative, and that the value 0 is attained only when $\boldsymbol{N}$ has equal row and column sums. The upper bound is proved by observing that

$$
\sum_{i=1}^{K}\left|n_{\cdot i}-n_{i \cdot}\right| \leq \sum_{i=1}^{K} n_{\cdot i}+\sum_{i=1}^{K} n_{i \cdot}=2 n .
$$

This bound is attained if and only if there is no cancelation between $n_{\cdot i}$ and $n_{i}$. for any $i$. This can happen, for instance, when $\boldsymbol{N}=n \boldsymbol{e}_{i} \boldsymbol{e}_{j}^{\prime}$ for some $i \neq j$.

Proof of Theorem 5.5. We work on the basis of the expansion (45) in lieu of (31). Proceeding as in the proof of Theorem 4.13, we have by virtue of IIR, NOM and ERI

$$
g\left(m_{1}, m_{2}, \ldots, m_{K^{3}(K-1) / 2}\right)=c_{1}\left(m_{1}+m_{2}+\cdots+m_{K^{3}(K-1) / 2}\right)
$$

for some constant $c_{1}$. Therefore, from IIR property of the measure $m$

$$
\begin{aligned}
& m\left(\sum_{i=1}^{K-1} \sum_{k=1}^{K-i} \sum_{j=1}^{K} \sum_{l=1}^{K} \gamma_{i j k l} \boldsymbol{F}_{i j k, l-j}\right) \\
& =g\left(m\left(\gamma_{1111} \boldsymbol{F}_{1110}\right), \ldots, m\left(\gamma_{K-1,111} \boldsymbol{F}_{K-1,110}\right), \ldots,\right. \\
& \left.\quad m\left(\gamma_{1 K 1 K} \boldsymbol{F}_{1 K 10}\right), \ldots, m\left(\gamma_{K-1, K 1 K} \boldsymbol{F}_{K-1, K 10}\right)\right) \\
& =c_{1} \sum_{i=1}^{K-1} \sum_{k=1}^{K-i} \sum_{j=1}^{K} \sum_{l=1}^{K} m\left(\gamma_{i j k l} \boldsymbol{F}_{i j k, l-j}\right) \quad \text { by IIR } \\
& =c_{1} \sum_{i=1}^{K-1} \sum_{k=1}^{K-i} \sum_{j=1}^{K} \sum_{l=1}^{K} \gamma_{i j k l} m\left(\boldsymbol{F}_{i j k, l-j}\right) \quad \text { by LH } \\
& =c_{1} c_{2} \sum_{i=1}^{K-1} \sum_{k=1}^{K-i} \sum_{j=1}^{K} \sum_{l=1}^{K} \gamma_{i j k l} \quad \text { by UM } \\
& =c_{1} c_{2} \sum_{i=1}^{K-1} \sum_{k=1}^{K-i} \sum_{j=1}^{K-1} \sum_{l=1}^{K-l}\left|\beta_{i j k l}\right| .
\end{aligned}
$$

for some constant $c_{2}$. This proves the necessity of the stated form of $m$. The sufficiency is easy to verify.

Proof of Theorem 5.6. The form of the measure follows directly from MIN and Theorem 5.5. The characterization of $\boldsymbol{N}$ for the attainment of the lower bound is also easy to show. 
In order to establish that the measure $M_{E N}$ is bounded from above by 1 , it is enough to show that for any transition matrix $\boldsymbol{N}$, the matrix $\boldsymbol{N}-\boldsymbol{N}_{0}-\boldsymbol{N}_{1}$ has a decomposition

$\sum_{i=1}^{K-1} \sum_{j=1}^{K-1} \sum_{k=1}^{K-i} \sum_{l=1}^{K-j} \beta_{i j k l} \boldsymbol{F}_{i j k l} \quad$ such that $\sum_{i=1}^{K-1} \sum_{j=1}^{K-1} \sum_{k=1}^{K-i} \sum_{l=1}^{K-j}\left|\beta_{i j k l}\right| \leq \frac{n(K-1)}{K}$.

To prove this, consider initially the simple special case $\boldsymbol{N}=n \boldsymbol{e}_{i} \boldsymbol{e}_{j}^{\prime}$ for some $i$ and $j$, so that $\boldsymbol{N}_{0}=n \boldsymbol{e}_{i} \boldsymbol{e}_{i}^{\prime}$ and

$$
\boldsymbol{N}_{1}=\frac{1}{K} \mathbf{1 1}^{\prime}\left(\boldsymbol{N}-\boldsymbol{N}_{0}\right)=\frac{n}{K} \mathbf{1} \boldsymbol{e}_{j}^{\prime}-\frac{n}{K} \mathbf{1} \boldsymbol{e}_{i}^{\prime}=\frac{n}{K} \sum_{k=1}^{K} \boldsymbol{e}_{k} \boldsymbol{e}_{j}^{\prime}-\frac{n}{K} \sum_{k=1}^{K} \boldsymbol{e}_{k} \boldsymbol{e}_{i}^{\prime} .
$$

It follows that

$$
\begin{aligned}
\boldsymbol{N}-\boldsymbol{N}_{0}-\boldsymbol{N}_{1} & =n \boldsymbol{e}_{i} \boldsymbol{e}_{j}^{\prime}-n \boldsymbol{e}_{i} \boldsymbol{e}_{i}^{\prime}-\frac{n}{K} \sum_{k=1}^{K} \boldsymbol{e}_{k} \boldsymbol{e}_{j}^{\prime}+\frac{n}{K} \sum_{k=1}^{K} \boldsymbol{e}_{k} \boldsymbol{e}_{i}^{\prime} \\
& = \begin{cases}\frac{n}{K} \sum_{k=1}^{i-1} \boldsymbol{F}_{k j, i-k, i-j}-\frac{n}{K} \sum_{k=i+1}^{K} \boldsymbol{F}_{i j, k-i, i-j} & \text { if } j<i, \\
0 & \text { if } j=i, \\
-\frac{n}{K} \sum_{k=1}^{i-1} \boldsymbol{F}_{k i, i-k, j-i}+\frac{n}{K} \sum_{k=i+1}^{K} \boldsymbol{F}_{i i, k-i, j-i} & \text { if } j>i .\end{cases}
\end{aligned}
$$

In each of the three cases, the sum of the absolute coefficients is less than or equal to $n(K-1) / K$.

Now suppose $\boldsymbol{N}=\sum_{i=1}^{K} \sum_{j=1}^{K} n_{i j} \boldsymbol{e}_{i} \boldsymbol{e}_{j}^{\prime}$, so that

$$
\boldsymbol{N}_{0}=\sum_{i=1}^{K} \sum_{j=1}^{K} n_{i j} \boldsymbol{e}_{i} \boldsymbol{e}_{i}^{\prime}, \quad \boldsymbol{N}_{1}=\sum_{i=1}^{K} \sum_{j=1}^{K} \frac{n_{i j}}{K}\left(\sum_{k=1}^{K} \boldsymbol{e}_{k} \boldsymbol{e}_{j}^{\prime}-\sum_{k=1}^{K} \boldsymbol{e}_{k} \boldsymbol{e}_{i}^{\prime}\right)
$$

and

$\boldsymbol{N}-\boldsymbol{N}_{0}-\boldsymbol{N}_{1}=\sum_{i=1}^{K} \sum_{j=1}^{K}\left(n_{i j} \boldsymbol{e}_{i} \boldsymbol{e}_{j}^{\prime}-n_{i j} \boldsymbol{e}_{i} \boldsymbol{e}_{i}^{\prime}-\frac{n_{i j}}{K} \sum_{k=1}^{K} \boldsymbol{e}_{k} \boldsymbol{e}_{j}^{\prime}+\frac{n_{i j}}{K} \sum_{k=1}^{K} \boldsymbol{e}_{k} \boldsymbol{e}_{i}^{\prime}\right)$.

As we have observed previously, each summand matrix has an expansion in terms of the $\boldsymbol{F}_{i j k l} \mathrm{~s}$, where the sum of the absolute coefficients is less than or equal to $n_{i j}(K-1) / K$. It follows that the expansion of $\boldsymbol{N}-\boldsymbol{N}_{0}-\boldsymbol{N}_{1}$ obtained by combining the expansions of the summands has sum of absolute coefficients less than or equal to $n(K-1) / K$. This proves that 1 is indeed an upper bound of $M_{E N}$. 
We have already seen that when $\boldsymbol{N}=n \boldsymbol{e}_{i} \boldsymbol{e}_{j}^{\prime}$, the matrix $\boldsymbol{N}-\boldsymbol{N}_{0}-\boldsymbol{N}_{1}$ has the expansion (53) with sum of absolute coefficients exactly equal to $n(K-1) / K$, as long as $j \neq i$. If there is another expansion with smaller sum of absolute coefficients, then the largest absolute value of any particular element of $\boldsymbol{N}-\boldsymbol{N}_{0}-\boldsymbol{N}_{1}$ must be smaller than $n(K-1) / K$. However, the $i$ th diagonal element of $\boldsymbol{N}-\boldsymbol{N}_{0}-\boldsymbol{N}_{1}$ is $-n(K-1) / K$. This contradiction proves that the bound is attained for this choice of $\boldsymbol{N}$.

For the other choice of $\boldsymbol{N}$ stated in the theorem,

$$
\boldsymbol{N}=\frac{n}{K} \sum_{i=1}^{K-1} \boldsymbol{e}_{i} \boldsymbol{e}_{i+1}^{\prime}+\frac{n}{K} \boldsymbol{e}_{K} \boldsymbol{e}_{1}^{\prime},
$$

so that

$$
\begin{aligned}
\boldsymbol{N}_{0} & =\frac{n}{K} \sum_{i=1}^{K-1} \boldsymbol{e}_{i} \boldsymbol{e}_{i}^{\prime}, \quad \boldsymbol{N}_{1}=\mathbf{0}, \\
\boldsymbol{N}-\boldsymbol{N}_{0}-\boldsymbol{N}_{1} & =\frac{n}{K}\left(\sum_{i=1}^{K-1} \boldsymbol{e}_{i} \boldsymbol{e}_{i+1}^{\prime}+\boldsymbol{e}_{K} \boldsymbol{e}_{1}^{\prime}-\sum_{i=1}^{K} \boldsymbol{e}_{i} \boldsymbol{e}_{i}^{\prime}\right) \\
& =\frac{n}{K} \sum_{i=1}^{K-1}\left(-\boldsymbol{e}_{i} \boldsymbol{e}_{i}^{\prime}+\boldsymbol{e}_{i}^{\prime} \boldsymbol{e}_{i+1}^{\prime}\right)+\frac{n}{K} \sum_{i=1}^{K-1}\left(\boldsymbol{e}_{K} \boldsymbol{e}_{i}^{\prime}-\boldsymbol{e}_{K} \boldsymbol{e}_{i+1}^{\prime}\right) \\
& =\frac{n}{K} \sum_{i=1}^{K-1}\left(-\boldsymbol{e}_{i} \boldsymbol{e}_{i}^{\prime}+\boldsymbol{e}_{i}^{\prime} \boldsymbol{e}_{i+1}^{\prime}+\boldsymbol{e}_{K} \boldsymbol{e}_{i}^{\prime}-\boldsymbol{e}_{K} \boldsymbol{e}_{i+1}^{\prime}\right) \\
& =\frac{n}{K} \sum_{j=1}^{K-1} \boldsymbol{F}_{i i 1, K-j} .
\end{aligned}
$$

It may be verified that the sum of the absolute coefficients of this expansion is $n(K-1) / K$.

There are $K-1$ elements of $\boldsymbol{N}-\boldsymbol{N}_{0}-\boldsymbol{N}_{1}$ that occur above the principal diagonal, and each of these elements is $-n / K$. Each of these positions should coincide with the top-right non-zero element of some $\boldsymbol{F}_{i j k l}$. (If it corresponds to another non-zero element of some $\boldsymbol{F}_{i j k l}$, then the corresponding top-right non-zero element would occur further away from the principal diagonal of $\boldsymbol{N}-\boldsymbol{N}_{0}-\boldsymbol{N}_{1}$, where there should be only zero elements.) Collecting the coefficients of these $K-1$ matrices of the type $\boldsymbol{F}_{i j k l}$, we conclude that the sum of absolute coefficients in an expansion of $\boldsymbol{N}-\boldsymbol{N}_{0}-\boldsymbol{N}_{1}$ must be greater than or equal to $n(K-1) / K$. Thus, the sum of the absolute coefficients obtained earlier indeed achieves the upper bound.

Proof of Theorem 5.7. It follows from Theorem 5.1 that the requisite measure is a scalar multiple of the minimum value of $\sum_{i=1}^{K} \sum_{j=1}^{K} \sum_{l=1}^{K} \tau_{i j l}$, where the minimum is over the coefficients of all possible expansions of $\boldsymbol{N}-\frac{1}{K} \boldsymbol{N} \mathbf{1 1}^{\prime}$ of the form (38). 
Let $A_{i}$ be the subset of the indices $j=1, \ldots, K$ such that $n_{i j}>n_{i} / K$, and $A_{i}^{c}$ be the complementary subset of those indices. Observe that

$$
\begin{aligned}
& \boldsymbol{e}_{i}^{\prime}\left(\boldsymbol{N}-\frac{1}{K} \boldsymbol{N} \mathbf{1 1 ^ { \prime }}\right)\left(\sum_{j \in A_{i}} \boldsymbol{e}_{j}\right) \\
& =\sum_{j \in A_{i}} \boldsymbol{e}_{i}^{\prime}\left(\boldsymbol{N}-\frac{1}{K} \boldsymbol{N 1 1}^{\prime}\right) \boldsymbol{e}_{j}=\sum_{j \in A_{i}}\left(n_{i j}-\frac{1}{K} n_{i} \cdot\right) \\
& =\frac{1}{2} \sum_{j \in A_{i}}\left(n_{i j}-\frac{1}{K} n_{i \cdot}\right)+\frac{1}{2} \sum_{j \in A_{i}^{c}}\left(\frac{1}{K} n_{i}-n_{i j}\right)=\frac{1}{2} \sum_{j=1}^{K} \mid n_{i j}-\frac{1}{K} n_{i} \cdot .
\end{aligned}
$$

Further, for any expansion of $\boldsymbol{N}-\frac{1}{K} \boldsymbol{N} \mathbf{1 1 ^ { \prime }}$ of the form (38), we can write

$$
\begin{aligned}
\boldsymbol{e}_{i}^{\prime}\left(\boldsymbol{N}-\frac{1}{K} \boldsymbol{N} \mathbf{1 1 ^ { \prime }}\right)\left(\sum_{j \in A_{i}} \boldsymbol{e}_{j}\right) \\
=\boldsymbol{e}_{i}^{\prime} \sum_{i^{\prime}=1}^{K} \sum_{j^{\prime}=1}^{K} \sum_{l=1}^{K} \tau_{i^{\prime} j^{\prime} l} \boldsymbol{e}_{i^{\prime}}\left(\boldsymbol{e}_{l}^{\prime}-\boldsymbol{e}_{j^{\prime}}\right)\left(\sum_{j \in A_{i}} \boldsymbol{e}_{j}\right) \\
=\sum_{j^{\prime}=1}^{K} \sum_{l=1}^{K} \tau_{i j^{\prime} l}\left(\boldsymbol{e}_{l}^{\prime}-\boldsymbol{e}_{j^{\prime}}\right)\left(\sum_{j \in A_{i}} \boldsymbol{e}_{j}\right) \\
=\sum_{j^{\prime}=1}^{K} \sum_{l \in A_{i}} \tau_{i j^{\prime} l}-\sum_{j^{\prime} \in A_{i}} \sum_{l=1}^{K} \tau_{i j^{\prime} l}=\sum_{j^{\prime} \in A_{i}^{c}} \sum_{l \in A_{i}} \tau_{i j^{\prime} l}-\sum_{j^{\prime} \in A_{i}} \sum_{l \in A_{i}^{c}} \tau_{i j^{\prime} l} \\
\leq \sum_{j^{\prime} \in A_{i}^{c}} \sum_{l \in A_{i}} \tau_{i j^{\prime} l}+\sum_{j^{\prime} \in A_{i}} \sum_{l \in A_{i}^{c}} \tau_{i j^{\prime} l} \leq \sum_{j=1}^{K} \sum_{l=1}^{K} \tau_{i j^{\prime} l},
\end{aligned}
$$

as all the $\tau_{i j l} \mathrm{~s}$ are nonnegative. Thus,

$$
\sum_{i=1}^{K} \sum_{j=1}^{K} \sum_{l=1}^{K} \tau_{i j^{\prime} l} \geq \frac{1}{2} \sum_{i=1}^{K} \sum_{j=1}^{K}\left|n_{i j}-\frac{n_{i}}{K}\right| .
$$

We can use a construction similar to the one used in the proof of Theorem 5.4, to obtain a special choice of (38) for which the bound is attained. This proves that the requisite measure is a scalar multiple of the right hand side of the above equation.

Now observe, for any fixed $i$,

$$
0 \leq \sum_{j=1}^{K}\left|n_{i j}-\frac{n_{i} \cdot}{K}\right| \leq n_{i} .
$$


The first equality holds with equality when $n_{i j}=n_{i} / K$. The second inequality holds with equality when, for a given $i$, half of the $n_{i j}$ are 0 and the other half are equal to $2 n_{i} / K$.

Proof of Theorem 5.8. In view of Theorem 5.3, we only have to minimize $\sum_{j=1}^{K} \sum_{l=1}^{K} \pi_{j l} \boldsymbol{L}_{j, l-j}$ over all possible representations of $\boldsymbol{N}_{S}=$ $\frac{1}{K} \mathbf{1 1}^{\prime}\left(\boldsymbol{N}-\frac{1}{K} \boldsymbol{N} \mathbf{1 1 ^ { \prime }}\right)$ of the form (44), and scale it suitably.

Let $A$ be the subset of indices $i=1, \ldots, K$ such that $n_{\cdot i}>n / K$, and $A^{c}$ be the complementary subset of those indices. Observe that

$$
\begin{aligned}
\mathbf{1}^{\prime} \boldsymbol{N}_{S}\left(\sum_{i \in A} \boldsymbol{e}_{i}\right) & =\mathbf{1}^{\prime} \frac{1}{K} \mathbf{1 1}^{\prime}\left(\boldsymbol{N}-\frac{1}{K} \boldsymbol{N} \mathbf{1} \mathbf{1}^{\prime}\right)\left(\sum_{i \in A} \boldsymbol{e}_{i}\right) \\
& =\sum_{j=1}^{N}\left(n_{\cdot j}-\frac{n}{K}\right) \boldsymbol{e}_{j}^{\prime}\left(\sum_{i \in A} \boldsymbol{e}_{i}\right)=\sum_{i \in A}\left(n_{\cdot i}-\frac{n}{K}\right) \\
& =\frac{1}{2} \sum_{i \in A}\left(n_{\cdot i}-\frac{n}{K}\right)+\frac{1}{2} \sum_{i \in A^{c}}\left(\frac{n}{K}-n_{\cdot i}\right)=\frac{1}{2} \sum_{i=1}^{K}\left|n_{\cdot i}-\frac{n}{K}\right| .
\end{aligned}
$$

For any expansion of $\boldsymbol{N}_{S}$ of the form (44), we can use the argument given in the proof of Theorem 5.4 to conclude that

$$
\sum_{j=1}^{K} \sum_{l=1}^{K} \pi_{j l} \geq \frac{1}{K} \mathbf{1}^{\prime} \boldsymbol{N}_{S}\left(\sum_{i \in A} \boldsymbol{e}_{i}\right)=\frac{1}{2 K} \sum_{i=1}^{K}\left|n_{\cdot i}-\frac{n}{K}\right| .
$$

The sharpness of this inequality can be established through the construction given in the proof of Theorem 5.4, with $n_{i}$. and $n_{j}$. replaced by $n / K$.

It is clear that the measure is nonnegative, and that the value 0 is attained only when $\boldsymbol{N}_{S}=\mathbf{0}$, i.e., when the destination distribution is uniform. In order to obtain the upper bound observe that

$$
\begin{aligned}
\sum_{i=1}^{K}\left|n \cdot i-\frac{n}{K}\right| & =\sum_{i \in A}\left(n_{\cdot i}-\frac{n}{K}\right)+\sum_{i \in A^{c}}\left(\frac{n}{K}-n \cdot i\right) \\
& \leq \sum_{i \in A}\left(n_{\cdot i}-\frac{n}{K}\right)+\sum_{i \in A^{c}} \frac{n}{K}=\sum_{i \in A} n_{\cdot i}-2 \sum_{i \in A} \frac{n}{K}+\sum_{i=1}^{K} \frac{n}{K} \\
& \leq \sum_{i \in A} n_{\cdot i}-\frac{2 n}{K}+n \leq \frac{2 n(K-1)}{K} .
\end{aligned}
$$

The first and the third inequalities hold with equality when $\sum_{i \in A} n_{\cdot i}=n$. The second inequality holds when $A$ is a singleton set. Thus, the maximum value of $\sum_{i=1}^{n}\left|n_{\cdot i}-n / K\right|$, achieved when the destination distribution has all the mass on a single state, is $2 n(K-1) / K$. 
Proof of Theorem 5.9. The given form of the measure follows from MIN and Theorem 5.5.

In order to establish that the measure $M_{I E N}$ is bounded from above by 1 , it is enough to show that for any transition matrix $\boldsymbol{N}$, the matrix $\boldsymbol{N}_{E}$ can be decomposed as

$$
\sum_{i=1}^{K-1} \sum_{j=1}^{K-1} \sum_{k=1}^{K-i} \sum_{l=1}^{K-j} \beta_{i j k l} \boldsymbol{F}_{i j k l}
$$

such that

$$
\sum_{i=1}^{K-1} \sum_{j=1}^{K-1} \sum_{k=1}^{K-i} \sum_{l=1}^{K-j}\left|\beta_{i j k l}\right| \leq \frac{n(K-1)^{2}}{K^{2}} .
$$

For this purpose, we rewrite $\boldsymbol{N}_{E}$ as

$$
\begin{aligned}
& \boldsymbol{N}_{E}=\left(I-\frac{1}{K} \mathbf{1 1}^{\prime}\right) \boldsymbol{N}\left(I-\frac{1}{K} \mathbf{1 1}^{\prime}\right) \\
& =\sum_{i=1}^{K} \sum_{j=1}^{K} n_{i j}\left(\boldsymbol{e}_{i} \boldsymbol{e}_{j}^{\prime}-\frac{1}{K} \mathbf{1} \boldsymbol{e}_{j}^{\prime}-\frac{1}{K} \boldsymbol{e}_{i} \mathbf{1}^{\prime}+\frac{1}{K^{2}} \mathbf{1 1}^{\prime}\right) \\
& =\sum_{i=1}^{K} \sum_{j=1}^{K} \sum_{k=1}^{K} \sum_{l=1}^{K} \frac{n_{i j}}{K^{2}}\left(\boldsymbol{e}_{i}-\boldsymbol{e}_{k}\right)\left(\boldsymbol{e}_{j}-\boldsymbol{e}_{l}\right)^{\prime} \\
& =-\sum_{i=2}^{K} \sum_{j=2}^{K} \sum_{k=1}^{i-1} \sum_{l=1}^{j-1} \frac{n_{i j}}{K^{2}} \boldsymbol{F}_{k l, i-k, j-l}+\sum_{i=2}^{K} \sum_{j=1}^{K-1} \sum_{k=1}^{i-1} \sum_{l=j+1}^{K} \frac{n_{i j}}{K^{2}} \boldsymbol{F}_{k j, i-k, l-j} \\
& +\sum_{i=1}^{K-1} \sum_{j=2}^{K} \sum_{k=i+1}^{K} \sum_{l=1}^{j-1} \frac{n_{i j}}{K^{2}} \boldsymbol{F}_{i l, k-i, j-l}-\sum_{i=1}^{K-1} \sum_{j=1}^{K-1} \sum_{k=i+1}^{K} \sum_{l=j+1}^{K} \frac{n_{i j}}{K^{2}} \boldsymbol{F}_{i j, k-i, l-j} \\
& =-\sum_{k=1}^{K-1} \sum_{l=1}^{K-1} \sum_{i=k+1}^{K} \sum_{j=l+1}^{K} \frac{n_{i j}}{K^{2}} \boldsymbol{F}_{k l, i-k, j-l} \\
& +\sum_{k=1}^{K-1} \sum_{j=1}^{K-1} \sum_{i=k+1}^{K} \sum_{l=j+1}^{K} \frac{n_{i j}}{K^{2}} \boldsymbol{F}_{k j, i-k, l-j} \\
& +\sum_{i=1}^{K-1} \sum_{l=1}^{K-1} \sum_{k=i+1}^{K} \sum_{j=l+1}^{K} \frac{n_{i j}}{K^{2}} \boldsymbol{F}_{i l, k-i, j-l} \\
& -\sum_{i=1}^{K-1} \sum_{j=1}^{K-1} \sum_{k=i+1}^{K} \sum_{l=j+1}^{K} \frac{n_{i j}}{K^{2}} \boldsymbol{F}_{i j, k-i, l-j} \\
& =\sum_{i=1}^{K-1} \sum_{j=1}^{K-1} \sum_{k=i+1}^{K} \sum_{l=j+1}^{K} \frac{-n_{k l}+n_{k j}+n_{i l}-n_{i j}}{K^{2}} \boldsymbol{F}_{i j, k-i, l-j} .
\end{aligned}
$$


The sum of absolute values of the coefficients of this expansion is

$$
\begin{aligned}
\sum_{i=1}^{K-1} \sum_{j=1}^{K-1} \sum_{k=i+1}^{K} \sum_{l=j+1}^{K} \frac{\left|-n_{k l}+n_{k j}+n_{i l}-n_{i j}\right|}{K^{2}} \\
\leq \sum_{i=1}^{K-1} \sum_{j=1}^{K-1} \sum_{k=i+1}^{K} \sum_{l=j+1}^{K} \frac{\left(n_{k l}+n_{k j}+n_{i l}+n_{i j}\right)}{K^{2}} \\
=\sum_{k=2}^{K} \sum_{l=2}^{K} \sum_{i=1}^{k-1} \sum_{j=1}^{l-1} \frac{n_{k l}}{K^{2}}+\sum_{k=2}^{K} \sum_{j=1}^{K-1} \sum_{i=1}^{k-1} \sum_{l=j+1}^{K} \frac{n_{k j}}{K^{2}} \\
\quad+\sum_{i=1}^{K-1} \sum_{l=2}^{K} \sum_{k=i+1}^{K} \sum_{j=1}^{l-1} \frac{n_{i l}}{K^{2}}+\sum_{i=1}^{K-1} \sum_{j=1}^{K-1} \sum_{k=i+1}^{K} \sum_{l=j+1}^{K} \frac{n_{i j}}{K^{2}} \\
=\sum_{k=1}^{K} \sum_{l=1}^{K}(k-1)(l-1) \frac{n_{k l}}{K^{2}}+\sum_{k=1}^{K} \sum_{j=1}^{K}(k-1)(K-j) \frac{n_{k j}}{K^{2}} \\
\quad+\sum_{i=1}^{K} \sum_{l=1}^{K}(K-i)(l-1) \frac{n_{i l}}{K^{2}}+\sum_{i=1}^{K} \sum_{j=1}^{K}(K-i)(K-j) \frac{n_{i j}}{K^{2}} \\
=\sum_{i=1}^{K} \sum_{j=1}^{K}(i-1)(K-1) \frac{n_{i j}}{K^{2}}+\sum_{i=1}^{K} \sum_{j=1}^{K}(K-i)(K-1) \frac{n_{i j}}{K^{2}}=\frac{n(K-1)^{2}}{K^{2}},
\end{aligned}
$$

which proves that 1 is indeed an upper bound of $M_{I E N}$.

We now show that this inequality holds with equality when $\boldsymbol{N}=n \boldsymbol{e}_{i} \boldsymbol{e}_{j}^{\prime}$ for some $i \neq j$. In such a case, we can follow the earlier derivation to write

$$
\boldsymbol{N}_{E}=\sum_{\substack{k=1 \\ k \neq i}}^{K} \sum_{\substack{l=1 \\ l \neq j}}^{K} \frac{n}{K^{2}}\left(\boldsymbol{e}_{i}-\boldsymbol{e}_{k}\right)\left(\boldsymbol{e}_{j}-\boldsymbol{e}_{l}\right)^{\prime}
$$

This expansion involves $(K-1)^{2}$ distinct elemental PEMs, each involving the $i j$ th position having the weight $n / K^{2}$ there. Therefore, the sum of absolute values of these coefficients is $n(K-1)^{2} / K^{2}$. A smaller sum cannot be achieved through an alternative expansion, since the $i j$ th element of $\boldsymbol{N}_{E}$ is $n(K-1)^{2} / K^{2}$.

It is clear that the lower bound is achieved if and only if $\boldsymbol{N}_{E}=\mathbf{0}$, which means the rows and columns of $\boldsymbol{N}$ are proportional to $\mathbf{1}$, that is, $\boldsymbol{N}=(n / K) \mathbf{1 1 ^ { \prime }}$.

\section{References}

1. Aaberge R., Atkinson A.B. and Königs S. (2018). From classes to copulas: wages, capital, and top incomes, Journal of Economic Inequality, 16 (2), 295-320. 
2. Atkinson A.B. (1981): The measurement of economic mobility, in Social Justice and Public Policy, MIT Press, Cambridge, MA.

3. Bartholomew, D.J. (1973): Stochastic Models for Social Processes, 2nd Edition, Wiley, London.

4. Bibby, J. (1975): Methods of measuring mobility, Quality and Quantity, 9, 107-136.

5. Breiger R.L. (1981): The social class structure of occupational mobility, American Journal of Sociology, 87 (3), 578-611.

6. Cambanis S., Simons G. and Stout W. (1976): Inequalities for E $k(X, Y)$ when the marginals are fixed, Zeitschrift für Wahrscheinlichkeitstheorie und Verwandte Gebiete, 36 (4), 285-294.

7. Chattopadhyay N., Dewanji A. and Roy B.K. (2019): Re-examining Bartholomew's index of social mobility: Some new derivations with statistical inference, Calcutta Statistical Association Bulletin, 71 (2), 99-112.

8. Chetty R., Grusky D., Hell M., Hendren N., Manduca R. and Narang J. (2017): The fading American dream: Trends in absolute income mobility since 1940, Science, 356 (6336), 398-406.

9. Cowel F.A. and Flachaire E. (2018): Measuring mobility, Quantitative Econometrics, 9, 865-901.

10. D'Agostino M. and Dardanoni V. (2009): The measurement of rank mobility, Journal of Economic Theory, 144, 1783-1803.

11. Dardanoni V. (1993): Measuring social mobility, Journal of Economic Theory, 61, 372-394.

12. Duncan O.D. (1979). How destination depends on origin in the occupational mobility table, American Journal of Sociology, 84 (4), 793-803.

13. Erikson R., Goldthorpe J.H. and Portocarero L. (1979): Intergenerational class mobility in three Western European countries: England, France and Sweden, British Journal of Sociology, 30, 415-440.

14. Ferretti C. and Ganugi P. (2013): A new mobility index for transition matrices, Statistical Methods and Applications, 22, 403-425.

15. Fields G.S. and Ok E.A. (1996): The meaning and measurement of income mobility, Journal of Economic Theory, 71 (2), 349-377.

16. Formby J.P., Smith W.J. and Zheng B. (2004): Mobility measurement, transition matrices and statistical inference, Journal of Econometrics, 120, 181-205. 
17. Glass D.V. and Hall J.R. (1954): Social mobility in Great Britain: A study of intergeneration changes in status, in: D.V. Glass, ed., Social mobility in Britain, The Free Press, Glencoe, IL, ch. VIII.

18. Goodman L.A. (1979): Simple models for the analysis of association in cross-classifications having ordered categories, Journal of the American Statistical Association, 74 (367), 537-552.

19. Hope K. (1982): Vertical and Nonvertical Class mobility in three countries, American Sociological Review, 47 (1), 99-113.

20. Markandya A. (1982): Intergenerational exchange mobility and economic welfare, European Economic Review, 17, 307-324.

21. Mitra T. and Ok E.A. (1998): The measurement of income mobility, Economic Theory, 12 (1), 77-102.

22. Prais S.J. (1955): Measuring social mobility, Journal of the Royal Statistical Society Series A, Part I, 118, 56-66.

23. Richey J. and Rosberg A. (2018): Decomposing economic mobility transition matrices, Journal of Applied Econometrics, 33, 91-108.

24. Ruiz-Castillo, J. (2004): The measurement of structural and exchange income mobility, Journal of Economic Inequality, 2, 219-228.

25. Shorrocks A.F. (1978): The measurement of mobility, Econometrica, 46 (5), 1013-1024.

26. Sobel M.E. (1983): Structural mobility, circulation mobility and the analysis of occupational mobility: A conceptual mismatch, American Sociological Review, 48 (5), 721-727.

27. Sobel M.E., Becker M.P. and Minick S.M. (1998): Origins, destinations, and association in occupational mobility, American Journal of Sociology, 104 (3), 687-721.

28. Sommers P.S. and Conlisk J. (1979): Eigenvalue immobility measures for Markov chains, Journal of Mathematical Sociology, 6, 253-276.

29. Swift A. (2004): Would Perfect Mobility be Perfect? European sociological Review, 20 (1), 1-11.

30. Tchen, A.H. (1980): Inequalities for distributions with given marginals, The Annals of Probability, 8, 814-827.

31. van de Gaer D., Schokkaert E. and Martinez M. (2001): Three meanings of intergenerational mobility, Economica, 68 (272), 519-537. 